BENTHIC MACROFAUNA AND ANCILLARY DATA FOR

SAN FRANCISCO BAY, CALIFORNIA, JANUARY TO NOVEMBER 1988

By Laurence E. Schemel, Allan Y. Ota, Jerry G. Harmon, Johnevan M. Shay, and Richard M. Adorador

U.S. GEOLOGICAL SURVEY

Open-File Report 89-596

Prepared in cooperation with the

CALIFORNIA STATE WATER RESOURCES CONTROL BOARD

$$
\begin{aligned}
& \infty \\
& \multirow{\sigma}{1}{} \\
& \sigma \\
& \stackrel{-1}{0} \\
& m
\end{aligned}
$$

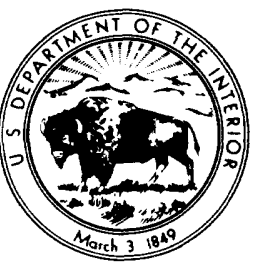

Sacramento, California 1990 


\section{DEPARTMENT OF THE INTERIOR}

\section{MANUEL LUJAN, JR., Secretary}

U.S. GEOLOGICAL SURVEY

Dallas L. Peck, Director

For additional information write to:

District Chief

U.S. Geological Survey

Federal Building, Room W-2234

2800 Cottage Way

Sacramento, CA 95825
Copies of this report may be purchased from:

U.S. Geological Survey

Books and Open-File Reports Section

Box 25425

Building 810, Federal Center

Denver, CO 80225 


\section{CONTENTS}

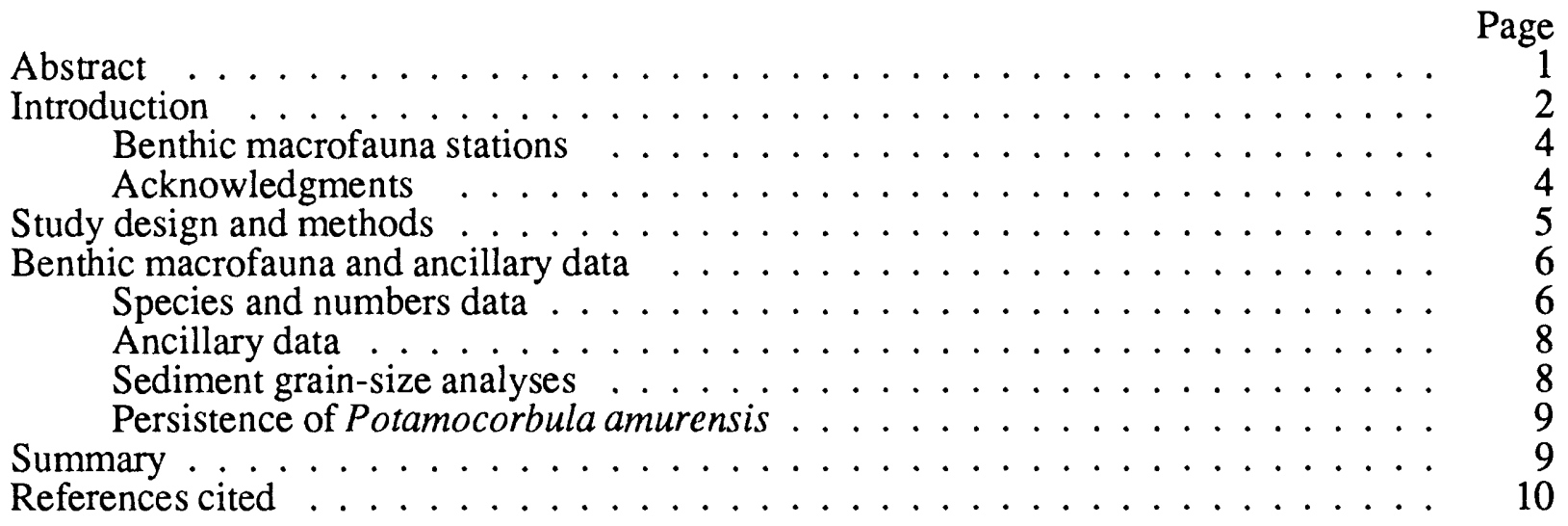

\section{ILLUSTRATION}

Figure 1. Map showing location of sampling stations in the Regional Effects Monitoring Program

Page

\section{TABLES}

Table 1. Regional Effects Monitoring Program stations . . . . . . . . . . . .

2. Cruise names and dates, $1988 \ldots \ldots \ldots \ldots$

3. Benthic macrofauna data . . . . . . . . . . . . . . . . . . . . . . . 11

4. Summary of benthic macrofauna data . . . . . . . . . . . . . . 51

5. Ancillary data . . . . . . . . . . . . . . . . . . . . . . 59

6. Summary of sediment grain-size data . . . . . . . . . . . . . . . . . 63

7. Cumulative number of species with respect to number of replicates . . . . . 64 


\section{CONVERSION FACTORS}

Metric units are used in this report. For readers who prefer inch-pound units, the conversion factors for the terms used in this report are listed below.

$\begin{array}{lcl}\text { Multiply } & \text { By } & \text { To obtain } \\ \mathrm{cm} \text { (centimeter) } & 0.3937 & \text { inch } \\ \mathrm{km} \text { (kilometer) } & 0.6214 & \text { mile } \\ \mathrm{m}_{\text {(meter) }} & 3.281 & \text { foot } \\ \mathrm{m}^{2} \text { (square meter) } & 10.76 & \text { square foot } \\ \mathrm{mm} \text { (millimeter) } & 0.03937 & \text { inch }\end{array}$

\section{TRADE NAMES}

The use of brand or trade names in this report is for identification purposes and does not imply endorsement by the U.S. Geological Survey. 


\title{
BENTHIC MACROFAUNA AND ANCILLARY DATA FOR \\ SAN FRANCISCO BAY, CALIFORNIA, JANUARY TO NOVEMBER 1988
}

\author{
By Laurence E. Schemel, Allan Y. Ota, Jerry G. Harmon, \\ Johnevan M. Shay, and Richard M. Adorador
}

\begin{abstract}
Benthic macrofauna and ancillary data were collected during 1988 as part of the U.S. Geological Survey Regional Effects Monitoring Program in San Francisco Bay, California. Data were collected during six cruises at 2-month intervals from January to November. Benthic macrofauna for identification and counting of species and sediments for size analysis were collected at eight stations. Temperature, salinity, and concentrations of dissolved oxygen and suspended sediment were measured at 12 stations. Salinity was measured at three stations that coincided with continuous monitoring stations.

The percentage of benthic macrofauna specimens that could be identified to the species level varied among sampling stations. Based on annual averages at each station, this percentage ranged from 60 to 95 percent. Three or fewer species represented from 25 to 75 percent of the total number of specimens at all stations. This percentage changed over time, as did the species. The annual average number of species per sample ranged from 4 to 21 . Stations in the southern bay were typically higher in number of species and number of specimens per sample than those in the northern bay. The newly introduced species of clam, Potamocorbula amurensis, persisted as an abundant species in the northern bay, and became more abundant at all stations in the southern bay except one.
\end{abstract}




\section{INTRODUCTION}

The U.S. Geological Survey began regular data collection for the Regional Effects Monitoring Program in 1987 and continued data collection for this program through 1988. The program was done in cooperation with the California State Water Resources Control Board, which developed the program as part of a plan for assessing effects of pollutants on the biological resources of San Francisco Bay. The major objective of the program is to detect long-term trends in selected biological and chemical properties by establishing a consistent and reliable data base. Data collected during the first few years will help evaluate variability caused by natural factors. This is necessary in order to identify long-term trends. This report presents data from the second year of the program.

Data collection during 1988 was limited to the benthic macrofauna and ancillary data components of the program. Measurements included in the ancillary data component were selected to characterize major changes in the physical and chemical environment that affect benthic macrofauna. Fifteen stations were sampled at 2-month intervals from January through November (table 1, fig. 1). Ancillary data, which consisted of measurements of salinity, temperature, and concentrations of dissolved oxygen and suspended particulate matter, were collected at 12 stations. These included the eight stations where samples were collected for benthic macrofauna and sediment grain-size analysis. Salinity was measured at three additional stations (stations 3, 7, and 9), where it is monitored continuously.

Table 1.-Regional Effects Monitoring Program stations

[Ancillary data were collected at all stations. An asterisk $(*)$ indicates stations where benthic macrofauna and sediment data also were collected]

\begin{tabular}{|c|c|c|c|}
\hline $\begin{array}{l}\text { Station No. } \\
\text { (fig. 1) }\end{array}$ & Station name & Latitude north & Longitude west \\
\hline$* 1$ & Palo Alto & $37^{\circ} 27.80^{\prime}$ & $122^{\circ} 04.90^{\prime}$ \\
\hline 2 & Redwood Creek & $37^{\circ} 33.25^{\prime}$ & $122^{\circ} 11.44^{\prime}$ \\
\hline 3 & San Mateo Bridge & $37^{\circ} 35.00^{\prime}$ & $122^{\circ} 22.00^{\prime}$ \\
\hline$* 4$ & Coyote Point & $37^{\circ} 36.30^{\prime}$ & $122^{\circ} 18.65$ \\
\hline$* 5$ & South Bay Deep & $37^{\circ} 41.20^{\prime}$ & $122^{\circ} 19.28^{\prime}$ \\
\hline$* 6$ & San Leandro & $37^{\circ} 39.57^{\prime}$ & $122^{\circ} 14.17$ \\
\hline 7 & Bay Bridge & $37^{\circ} 47.50^{\prime}$ & $122^{\circ} 23.00^{\prime}$ \\
\hline$* 8$ & Berkeley & $37^{\circ} 52.43^{\prime}$ & $122^{\circ} 21.20^{\prime}$ \\
\hline 9 & Point San Pablo & $37^{\circ} 57.87^{\prime}$ & $122^{\circ} 25.72^{\prime}$ \\
\hline$* 10$ & San Pablo Shallow & $38^{\circ} 03.75^{\prime}$ & $122^{\circ} 24.40^{\prime}$ \\
\hline$* 11$ & San Pablo Deep & $38^{\circ} 02.67^{\prime}$ & $122^{\circ} 18.93^{\prime}$ \\
\hline 12 & Suisun Bay & $38^{\circ} 03.12^{\prime}$ & $122^{\circ} 06.63^{\prime}$ \\
\hline *13 & Grizzly Bay & $38^{\circ} 06.97^{\prime}$ & $122^{\circ} 02.33^{\prime}$ \\
\hline 14 & Honker Bay & $38^{\circ} 03.80^{\prime}$ & $121^{\circ} 58.00^{\prime}$ \\
\hline 15 & Chipps Island & $38^{\circ} 02.80^{\prime}$ & $121^{\circ} 55.00^{\prime}$ \\
\hline
\end{tabular}




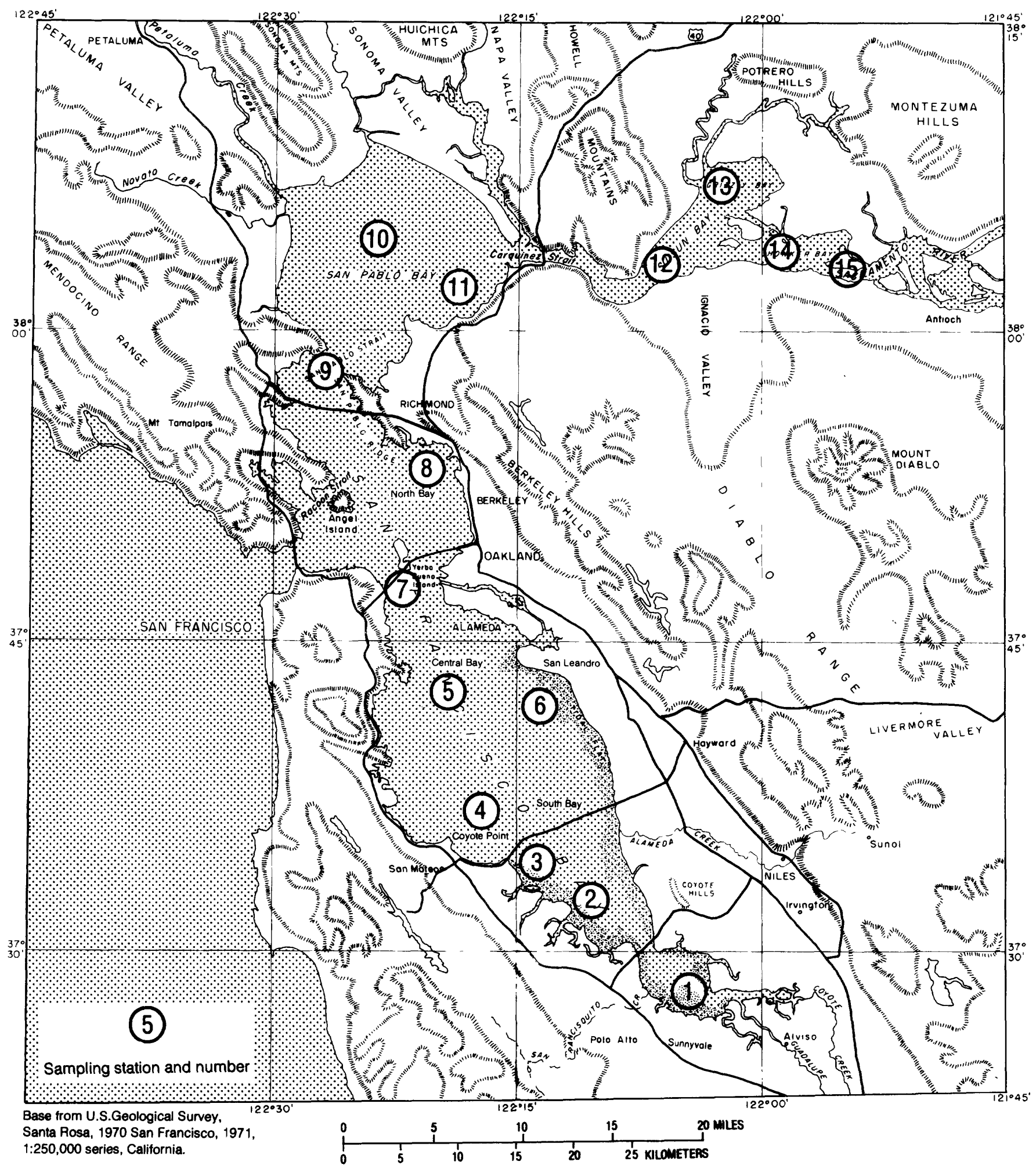

Figure 1.- Location of sampling stations in the Regional Effects Monitoring Program. 


\section{Benthic Macrofauna Stations}

Palo Alto (Station 1) is in shallow water on the west side of southern San Francisco Bay, south of the Dumbarton Bridge in a large area that appears relatively uniform in the type of substrate. This station is about $1.5 \mathrm{~km}$ east of a transect of three intertidal stations that were studied by the U.S. Geological Survey for changes in benthic macrofauna community structure for about 14 years (Nichols and Thompson, 1985a).

Coyote Point (Station 4) is in shallow water on the west side of the deep channel in southern San Francisco Bay, north of the San Mateo Bridge. The National Oceanic and Atmospheric Administration Status and Trends program occupies a station annually that is north of this station near Hunters Point.

South Bay Deep (Station 5) is in deep water east of the dredged channel in southern San Francisco Bay. This station is about $2.7 \mathrm{~km}$ west of a major municipal waste outfall. Finding a suitable location for a deep water station in the southern bay is difficult because of dredging, locations of pipelines and anchorages, and major municipal waste outfalls.

San Leandro (Station 6) is in shallow water on the east side of southern San Francisco Bay, north of the San Mateo Bridge. This station is in a large area of shell debris, and replicate samples exhibit greater variability in substrate type than at most other stations. For example, some samples show exposed shell debris, whereas others are primarily mud.

Berkeley (Station 8) is in a broad, relatively uniform area in central San Francisco Bay, west of Berkeley. This station is southeast of a Status and Trends station in the area of the Southampton Shoal channel, and is north of a Mussel Watch station and a major municipal waste outfall. This station represents the most typically marine environment sampled by the program.

San Pablo Shallow (Station 10) is in a broad, relatively uniform area in shallow water on the west side of the deep channel in San Pablo Bay. It is north of a deeper water station that was sampled by the National Oceanic and Atmospheric Administration in their Triad studies.

San Pablo Deep (Station 11) is on the east side of the deep channel west of the Status and Trends station. This deep water station in San Pablo Bay typically exhibited variability in substrate type and the presence of unconsolidated debris. In spite of these problems, this station was sampled during 1988 so that the persistence of the newly introduced clam, Potamocorbula amurensis, could be monitored.

Grizzly Bay (Station 13) is adjacent to a station that has been sampled monthly since 1980 by the California Department of Water Resources as part of D1485 monitoring. Grizzly Bay provides overlap between the two programs. The Department of Water Resources program extends landward to locations in the delta of the Sacramento and San Joaquin Rivers.

\section{Acknowledgments}

The authors wish to thank Brian T. Yost for assistance with data collection and processing and Janet K. Thompson and Frederic H. Nichols for much helpful advice and assistance with quality control of the benthic macrofauna data. 


\section{STUDY DESIGN AND METHODS}

Samples for benthic macrofauna and ancillary data were collected during six cruises on the U.S. Geological Survey Research Vessel, R/V Saul E. Rantz (table 2). The dates of the sampling cruises were selected to fall within the neap tides of the month. At these times when tidal currents were weak, the wire angle was nearly vertical, the benthic sampler landed squarely on the substrate, and the area that was sampled varied little among the replicates. Weather was a major factor, particularly wind, which created swells, and consequently boat motion during sampling. Under these conditions, the sampler could not penetrate the substrate or operate correctly. Consequently, no samples could be collected until the wind subsided.
Table 2.-Cruise names and dates, 1988

\begin{tabular}{ll}
\hline Cruise name & \multicolumn{1}{c}{ Sampling dates } \\
\hline & \\
JAN88 & January 11-14 \\
MAR88 & March 7, 8, 14, 15 \\
MAY88 & May 23, 24, 25, 26, 31 \\
JUL88 & July 20, 21, 25, 26, 27, 28 \\
SEP88 & September 12-14, 15 \\
NOV88 & October $31-$ November 2, 3
\end{tabular}

Samples were usually collected at stations 12 through 15 on the first day, at stations 7 through 11 on the second day, at stations 2 through 6 on the third day, and at station 1 on the fourth day. Exact locations of the stations were selected so that they could be relocated easily by the use of radar and navigational aids. Station locations were confirmed by loran c.

Benthic macrofauna were collected with a modified Van Veen, which sampled an area of 0.05 $\mathrm{m}^{2}$. Depth of penetration of the sampler varied with substrate type. Soft substrates were sampled to a depth of 15 to $18 \mathrm{~cm}$, whereas penetration of hard substrates was often limited to 7 to $10 \mathrm{~cm}$. Six samples were collected at each station; five were for benthic macrofauna analysis and one was for sediment grain-size analysis. The sample for grain-size analysis was subsampled from the consolidated sediment just below the surface layer but above the redox discontinuity.

Benthic macrofauna samples were washed on a $0.5-\mathrm{mm}$ mesh screen to remove the fine sediment. Macrofauna and debris were transferred to plastic jars, where organisms were relaxed with propylene phenoxytol, then preserved in a buffered formalin solution (10 percent). After 4 to 7 days, samples were washed to remove the formalin solution, then transferred to a 70 percent solution of ethyl alcohol.

Water samples were collected at eight stations where samples for benthic macrofauna were collected and at seven additional stations. The seven additional stations enabled better characterization of the salinity field and chemical-physical environment of the estuary. Three of these additional stations $(3,7$, and 9$)$ were at locations where temperature and specific conductance were monitored continuously. These monitors were installed by the California Department of Water Resources and are now operated by the U.S. Geological Survey. Samples collected at these stations during the Regional Effects Monitoring cruises provided a link between Regional Effects Monitoring data and data from the monitors. Data from the monitors are needed to evaluate changes in the environment of the estuary between Regional Effects Monitoring cruises.

Samples for salinity analysis were collected at the three locations with continuous monitors. Samples were collected at the remaining 12 stations as part of the ancillary-data collection. Near-surface water $(1 \mathrm{~m})$ was sampled with a Niskin Bottle from which two dissolved-oxygen samples, one salinity sample, and a sample for suspended particulate matter were drawn. When water depths exceeded about $3 \mathrm{~m}$, a deeper water sample, about $1 \mathrm{~m}$ above the bottom, was collected in an identical manner. 
Samples for dissolved oxygen were collected in glass-stoppered iodine flasks, immediately preserved with $1-\mathrm{mL}$ aliquots of manganous sulfate reagent and alkaline iodide-azide reagent, then shaken. Samples were kept cool and out of direct sunlight. A small quantity of distilled water was placed in the flange of each flask to ensure the seal of the ground glass stopper. Laboratory and field tests indicated that the titration of the dissolved- oxygen samples could be delayed for at least 1 week when the above procedures were followed.

Salinity was determined with a high precision laboratory salinometer calibrated with standard seawater. Salinity was reported without the traditional units, in accordance with the practical salinity scale of 1978 (Lewis, 1980). Dissolved oxygen was determined by the Winkler titration method (azide modification; American Public Health Association, 1985). Suspended particulate matter in a known volume of sample was collected on a tared membrane filter, dried, and weighed to determine the concentration. Sediment samples were analyzed for grain size by the wet-sieve and hydrometer method (Guy, 1969).

Kinnetic Laboratories, Inc. of Carlsbad, California, was contracted to provide identifications and counts of macrofaunal species. Identifications were made to the species level or the lowest possible taxon. U.S. Geological Survey experts in the field of benthic ecology, Janet Thompson and Frederic Nichols, provided a final check on the quality of the benthic macrofauna data.

\section{BENTHIC MACROFAUNA AND ANCILLARY DATA}

Identifications of benthic macrofauna to the lowest possible taxon, usually genus and species, are shown in table 3 . Table 4 is a summary of these data showing the numbers of taxanomic entries, species, and individuals for each replicate, station, and date. Ancillary data are presented in table 5 , and sediment grain-size data are presented in table 6.

\section{Species and Numbers Data}

The data presented in table 3 can be summarized by various biological indexes. The number of species and the number of individuals within each species are key biological properties that commonly are used in the calculation of indexes, such as diversity indexes, which summarize the community characteristics. Selection and use of indexes to summarize benthic macrofauna data is a subject of controversy among ecologists, but they generally agree on the need for sample replication to provide results that are statistically valid (Green, 1979). In this study, a level of replication was selected that, based on the experiences of previous investigators, should collect most of the species and provide sufficiently accurate estimates of their abundances.

Five replicates were collected at each station, but on two occasions only four of the replicates passed our quality control (see table 3). The cumulative number of species collected with respect to the number of replicates is shown for each station and sampling cruise in table 7. Almost all species that were abundant in high or moderate densities (numbers per unit area) were present in all five replicates. When specimens of a species were collected only in the fourth or fifth replicate or both, density of that species was typically low (only one or two individuals from all five replicates). Only 3 of the 183 species that were collected only in the fourth or fifth replicate or both had densities equal to or exceeding an average of one specimen per replicate. Relative to the total numbers of species collected in all five replicates, an average of about 80 percent were collected by the third replicate and about 90 percent were collected by the fourth replicate at all of the stations. The percentages were highest at Palo Alto, where an average of 95 percent of the species were collected by the third replicate, and they were lowest at San Pablo Deep, where an average of 74 percent of the species were collected by the third replicate and 85 percent by the fourth replicate. 
Identification of benthic macrofauna to the species level is necessary for the characterization of the community structure and for the calculation of some indexes. For various reasons, some individuals will not be identified to the species level. The percentage of the total individuals that was represented by specimens that were identified to the species level varied greatly among stations in this study. On the average, about 95 percent of the individuals could be identified to the species level at Palo Alto. This percentage was about 90 percent at Coyote Point, about 80 percent at South Bay Deep, about 70 percent at San Leandro, and about 60 percent at Berkeley. In the northern bay, the greatest percentage of individuals usually were identified to the species level at Grizzly Bay (about 95 percent), followed by about 90 percent at San Pablo Shallow and about 80 percent at San Pablo Deep. In most specimens, lower percentages were the result of moderate to high numbers of oligochaetes or nematodes, or both, and other organisms including an unidentified authozoan at South Bay Deep and Phoronsis sp(p) at Berkeley.

In general, the abundances of one or two species (dominant species) represented a large percentage of the total number of individuals, followed by as much as three or four species with abundances of one-half or less those of the dominant species. The percentage of the total abundance represented by the dominant species varied with time, as did the species that were dominant. Fifty to more than 75 percent of the individuals at Palo Alto were represented by two species, Gemma gemma and Ampelisca abdita. One-half of the individuals at Coyote Point and South Bay Deep were represented by two crustaceans, Ampelisca abdita and Corophium sp(a), and two polychaetes, Exogone lourei and Asychis elongata. On most dates, one of these species represented 25 to 50 percent of the individuals. At San Leandro, 25 percent of the individuals were represented by the mollusk, Musculista senhousia, or the crustacean, Ampelisca abdita. In general, Berkeley exhibited a large number of species with relatively low densities (see below). However, a single crustacean, Ampelisca abdita or Corophium sp(a), represented more than one-half of the individuals during three of the six sampling times. Fifty to more than 75 percent of the individuals at San Pablo Deep were represented by the newly introduced species of mollusk, Potamocorbula amurensis, or the crustacean, Corophium sp(a). About 50 percent of the individuals at San Pablo Shallow were represented by Ampelisca abdita during three sampling times and by the newly introduced mollusk, Potamocorbula amurensis, during May, July, and September. Potamocorbula amurensis represented more than 75 percent of the individuals at Grizzly Bay during all sampling dates.

Differences in the mean numbers of species and the mean numbers of individuals were observed among the stations. These differences were generally consistent over time, so the following discussion was based on annual means for numbers of species and individuals per sample. Lowest numbers of species ( 4 to 6 ) were observed at Grizzly Bay and at San Pablo Deep. Moderate numbers of species (12 to 15) were observed at Berkeley, San Pablo Shallow, and San Leandro. High numbers of species (18 to 21) were observed at Coyote Point, Palo Alto, and South Bay Deep. Palo Alto exhibited the greatest number of individuals, more than a factor of three higher than the next highest stations, Coyote Point, San Leandro, and San Pablo Shallow. Lowest numbers of individuals were observed at San Pablo Deep, followed by Grizzly Bay, Berkeley, and South Bay Deep. The mean number of individuals at South Bay Deep was about one-half that at San Leandro or Coyote Point.

Differences in the numbers of species and numbers of individuals among the replicates are expressed here as the coefficient of variation, the standard deviation divided by the mean. The values were averaged for the six sampling dates to provide a mean coefficient of variation for comparisons among stations. Mean coefficient of variation for species was lower than mean coefficient of variation for individuals at all stations. The lowest mean coefficient of variation for species and individuals were observed in the southern bay at Palo Alto ( 0.10 and 0.17 , respectively), and in the northern bay at San Pablo Shallow (0.12 and 0.24, respectively). Low values also were typical at Coyote Point, Grizzly Bay, and South Bay Deep. The highest mean coefficient of variation for species was at San Pablo Deep (0.41), and highest mean coefficient of variation for individuals was at Berkeley (0.70). Coefficients of variation among replicates were consistently high for these two stations, although one or two outlier values were often the cause of a high coefficient of variation at any one sampling time. 
A major goal of the Regional Effects Monitoring program is the detection of long-term trends in the abundances and community structures of the benthic macrofauna. Analysis of the data for trends is not possible at this time. Data from many years are required to distinguish trends, primarily because natural variability results from annual and semiannual cycles of climate and reproduction (Nichols and Pamatmat, 1988).

\section{Ancillary Data}

Ancillary data collected with the benthic macrofauna samples characterize changes in environmental conditions in the estuary. Northern California experienced a second consecutive year of less-than-normal rainfall during 1988. Rainfall is seasonal in northern California, with most occurring from late autumn through early spring. Inflow of fresh water from runoff is a major factor affecting salinity in San Francisco Bay (Dedini and others, 1981). Dilution by freshwater inflow generally results in lower than average salinities through about May or June. Effects of freshwater inflow on salinity vary with location in the estuary. The upper reaches of northern San Francisco Bay, including Grizzly Bay, are most affected by seasonal variations in freshwater inflow from the Sacramento River and San Joaquin River. Similarly, salinity at Palo Alto can be greatly reduced by runoff from local streams.

Salinities measured at Grizzly Bay and Palo Alto during the winter and spring of 1988 were greater than values measured during 1980, a year with a normal amount of freshwater inflow (Dedini and others, 1981). The effect of low levels of freshwater inflow on salinity was greater at Grizzly Bay, where values ranged from 4.1 in January to 12.5 in July 1988 . In contrast, salinities were less than 1 from January through May 1980, then reached a maximum value of about 7.6 during September (Dedini and others, 1981). Previous investigations have shown that similar increases in the salinity at Grizzly Bay affect the species composition of the benthic macrofauna (Nichols and Thompson, 1985b; Nichols and Pamatmat, 1988).

Dissolved oxygen was saturated at levels exceeding 80 percent in 75 of the 76 samples. Dissolved oxygen was above 90 percent saturation in 57 of the samples, and exceeded saturation in 5 samples that were taken in southern San Francisco Bay during March.

\section{$\underline{\text { Sediment Grain-Size Analyses }}$}

Substrates at all the stations sampled for benthic macrofauna are classified as soft bottom substrates. San Leandro differed somewhat from the others because a large amount of shell debris was present in the mud. The shell debris generally was larger than $1 \mathrm{~mm}$, and as a consequence it was not quantified in the sediment grain-size analysis.

Differences among the sediment grain sizes at the stations are shown in table 6. San Leandro, Coyote Point, Grizzly Bay, and Palo Alto were similar in that about 50 percent of the sediment typically was finer than $0.004 \mathrm{~mm}$. San Pablo Shallow and Berkeley were similar in that about 50 percent of the sediment typically was finer than $0.008 \mathrm{~mm}$, although grain sizes were somewhat more variable at Berkeley. The two deep stations, San Pablo Deep and South Bay Deep, were distinctively different from the others in that they exhibited greater variability in grain-size distributions and the sediments generally were coarser (larger grain size). Greatest variability in sediment grain size was observed at San Pablo Deep. The results from the sediment grain-size analyses for 1988 were consistent with those for 1987 (Schemel and others, 1988). 


\section{Persistence of Potamocorbula amurensis}

By reviewing available taxanomic literature, Dr. James T. Carlton of the University of Oregon has determined that the probable genus and species of the newly introduced clam is Potamocorbula amurensis. It is possible, however, that synonyms might exist.

The spread of the new species of clam shortly after its introduction was documented during the first year of sampling of this program (Schemel and others, 1988). Data collected during 1988 show that this new species persisted as a dominant species in northern San Francisco Bay and became more abundant in areas of southern San Francisco Bay. Because of low levels of freshwater inflow during 1988, Potamocorbula amurensis has not experienced a normal seasonal range of environmental conditions at locations in the northern and southern bays.

Greatest densities (abundances per unit area) of the new clam during 1987 were observed at Grizzly Bay and San Pablo Deep (Schemel and others, 1988). Although densities at these locations during 1988 were lower than the maximum densities during 1987, examinations in the laboratory have indicated that specimens generally were larger in size during 1988 (J.K. Thompson, U.S. Geological Survey, oral commun., 1988). Specimens were found in all replicates collected at Grizzly Bay and in all but one replicate collected at San Pablo Deep during 1988. Densities of the new clam during 1988 were greater than those during 1987 at the other stations with the exception of San Leandro, where no specimens were collected during 1987 or 1988. Lowest densities, typically about one specimen or less per sample, were found at South Bay Deep and Berkeley. Densities increased during 1988 at Coyote Point and Palo Alto. Specimens were found in all but one replicate collected at Coyote Point, where densities averaged about 40 specimens per sample during September and November. Specimens were found in all replicates collected at Palo Alto beginning in May. Average density increased to a maximum value of 65 specimens per sample in November at Palo Alto. Specimens of the new clam have been found in every replicate collected at San Pablo Shallow since July 1987. Average density at San Pablo Shallow reached a maximum of 455 specimens per sample in May 1988.

\section{SUMMARY}

Sampling was continued for the Regional Effects Monitoring Program in San Francisco Bay during 1988. Samples for benthic macrofauna, sediment grain size, and ancillary data were collected during six cruises at 2-month intervals beginning in January. Benthic macrofauna were identified to the species level or the lowest possible taxon and counted. Eight stations were sampled for benthic macrofauna and sediment grain size during each cruise. At each station, five replicate samples were collected for benthic macrofauna, and one sample was collected for sediment grain-size analysis. Ancillary data, consisting of salinity, temperature, and dissolved-oxygen and suspended-sediment concentrations, were collected at 12 stations, including the 8 stations where benthic macrofauna samples were collected. Salinity was measured at three stations that coincided with continuous water-quality monitors. Benthic macrofauna data showed the persistence of the newly introduced clam, Potamocorbula amurensis, at stations in the northern bay, and increasing densities at all stations except San Leandro in the southern bay. 


\section{REFERENCES CITED}

American Public Health Association, 1985, Standard methods for the examination of water and wastewater, 16th ed.: Washington, D.C., p. 413-419.

Dedini, L.A., Schemel, L.E., and Tembreull, M.A., 1981, Salinity and temperature measurements in San Francisco Bay waters, 1980: U.S. Geological Survey Open-File Report 82-125, 130 p.

Green, R.H., 1979, Sampling design and statistical methods for environmental biologists: New York, John Wiley, 257 p.

Guy, H.P., 1969, Laboratory theory and methods for sediment analysis: U.S. Geological Survey Techniques of Water-Resources Investigations, book 5, chap. C1, 58 p.

Lewis, E.L., 1980, The practical salinity scale of 1978 and its antecedents: Journal of Oceanic Engineering, v. OE-5, no. 1, p. 3-8.

Nichols, F.H., and Pamatmat, M.M., 1988, The ecology of the soft-bottom benthos of San Francisco Bay: a community profile: U.S. Fish and Wildlife Service Biological Report 85(7.19), 73 p.

Nichols, F.H. and Thompson, J.K., 1985a, Persistence of an introduced mudflat community in South San Francisco Bay, California: Marine Ecology-Progress Series, v. 24, p. 83-97.

1985b, Timescales of change in the San Francisco Bay benthos: Hydrobiologia, v. 129, p. $121-138$.

Schemel, L.E., Ota, A.Y., Harmon, J.G., Shay, J.M., and Adorador, R.M., 1988, Benthic macrofauna and ancillary data for San Francisco Bay, California, March to November 1987: U.S. Geological Survey Open-File Report 88-192, 73 p. 
Table 3.-Benthic macrofauna data

\begin{tabular}{lcccccc}
\hline \multirow{2}{*}{ Taxonomic entry } & Kinnetic & \multicolumn{5}{c}{ Individuals } \\
\cline { 2 - 6 } & Laboratory & \multicolumn{4}{c}{ Replicate number } \\
\cline { 2 - 6 } & code & 1 & 2 & 3 & 4 & 5 \\
\hline
\end{tabular}

$\underline{\text { Berkeley }}$

Sampling date: January 12,1988

Amaeana occidentalis ............44810001

Ampelisca abdita ..................5275504

Anaitides longipes ..............4810505

Asychis elongata ...............4810565

Cerebratulus sp(p). ..............4000014

Corophium alienense ..............55275287

Cryptomya californica ............5540155

Edwardsia sipunculoides ............3730022

Edwardsiidae, unident. . . . . . . . . . . . 3730045

Euchone limnicola ...............4810255

Exogone lourei .................4810066

Glycinde polygnatha ..............4810496

Harmothoe lunulata ...............4810689

Hemigrapsus oregonensis . . . . . . . . . 5286092

Hesperonoe $\mathrm{sp}(\mathrm{p}) . . . \ldots . . . . . . . . .4810090$

Leitoscoloplos pugettensis ..........4810516

Leptochelia dubia .................5264038

Lineidae, unident. . ..............4000038

Macoma sp(p)..................5540105

Musculista senhousia .............5540401

Mysella sp(p). ..................5540137

Mysella sp. (A) (SCAMIT) ............55540212

Mytilus edulis ..................5540024

Natantia, unident. . . . . . . . . . . . 5286111

Nematodes, unident. . . . . . . . . . . . .4500001

Nephtys ferruginea ..............44810706

Nephtys sp(p). ..................4810579

Oligochaete, unident. .............44880001

Philine sp. (A) (SCAMIT) ........... 5570240

Pholoe glabra ...................4810442

Phoronis sp(p). ................5700002

Potamocorbula amurensis ...........5540214

Protothaca staminea ...............5540035

Pseudopolydora kempi .............4810640

Pseudopolydora paucibranchiata . . . . . 48810347

Sarsiella zostericola ..............55220091

Schistomeringos rudolphi ...........4810354

Scleroplax granulata ..............55286519

Sphaerosyllis californiensis .........4810272

Stylatula sp(p)................... 3730044

Tapes japonica .................5540158

\begin{tabular}{|c|c|c|c|}
\hline 4 & 4 & 4 & 3 \\
\hline 103 & 268 & 217 & 73 \\
\hline 0 & 1 & 1 & 1 \\
\hline 0 & 0 & 4 & 2 \\
\hline 3 & 0 & 0 & 0 \\
\hline 7 & 10 & 4 & 1 \\
\hline 1 & 1 & 0 & 0 \\
\hline 0 & 1 & 1 & 4 \\
\hline 1 & 0 & 0 & 0 \\
\hline 1 & 3 & 4 & 0 \\
\hline 2 & 7 & 8 & 2 \\
\hline 2 & 5 & 5 & 10 \\
\hline 0 & 1 & 0 & 0 \\
\hline 0 & 0 & 1 & 0 \\
\hline 0 & 0 & 1 & 1 \\
\hline 3 & 3 & 4 & 3 \\
\hline 1 & 5 & 8 & 1 \\
\hline 0 & 1 & 0 & 0 \\
\hline 0 & 0 & 1 & 0 \\
\hline 1 & 0 & 2 & 0 \\
\hline 3 & 0 & 0 & 0 \\
\hline 3 & 0 & 2 & 1 \\
\hline 1 & 0 & 1 & 0 \\
\hline 0 & 0 & 0 & 0 \\
\hline 3 & 64 & 37 & 0 \\
\hline 0 & 1 & 1 & 0 \\
\hline 0 & 0 & 0 & 1 \\
\hline 3 & 39 & 3 & 2 \\
\hline 2 & 0 & 0 & 1 \\
\hline 0 & 0 & 1 & 0 \\
\hline 340 & 10 & 0 & 0 \\
\hline 0 & 1 & 0 & 0 \\
\hline 0 & 0 & 1 & 0 \\
\hline 0 & 1 & 1 & 0 \\
\hline 0 & 1 & 2 & 1 \\
\hline 0 & 3 & 0 & 0 \\
\hline 2 & 0 & 0 & 0 \\
\hline 1 & 0 & 0 & 0 \\
\hline 1 & 66 & 5 & 1 \\
\hline 0 & 2 & 0 & 1 \\
\hline 0 & 1 & 0 & 0 \\
\hline
\end{tabular}


Table 3.-Benthic macrofauna data-Continued

\begin{tabular}{lccccc}
\hline \multirow{2}{*}{ Taxonomic entry } & Kinnetic & \multicolumn{5}{c}{ Individuals } \\
\cline { 3 - 6 } & Laboratory & \multicolumn{4}{c}{ Replicate number } \\
\cline { 3 - 6 } & code & 1 & 2 & 3 & 4 \\
\hline
\end{tabular}

\section{Berkeley}

Sampling date: March 8, 1988

Amaeana occidentalis .............4810001

Ampelisca abdita .................5275504

Amphiodia digitata $(j u v) \ldots \ldots \ldots \ldots . . .5930026$

Amphiuridae, unident. . ............ 5930032

Anthozoan, unident. ............... 3730010

Asychis elongata ................4810565

Campanularidae, unident. ............3710039

Chaetognatha .................6.6200000

Cirriformia spirabrancha .......... 4810854

Corophium alienense . . . . . . . . . . . . .5275287

Corophium sp(p). . . . . . . . . . . . . . . . 5275098

Cryptomya californica .............5540155

Decapod larvae ..................55286045

Edwardsia sipunculoides . . . . . . . . . 3730022

Euchone limnicola ...............4810255

Exogone lourei ................4810066

Gemma gemma ..................5540400

Glycinde polygnatha ...............4810496

Hesperonoe $\mathrm{sp}(\mathrm{p}) . . . \ldots . . . . . . . . .44810090$

Leitoscoloplos pugettensis . .........4810516

Leptochelia dubia .................5264038

Leucon subnasica ...............55263012

Lightiella serendipita .............5204001

Lineidae, unident. . . . . . . . . . . . . 4000038

Mediomastus sp. ...............44810303

Modiolus sp(p). ..................5540409

Mysella sp(p). .................55540137

Mysella sp. (A) (SCAMIT) . . . . . . . . . 5540212

Nematodes, unident. ..............4500001

Nephtys cornuta franciscana . . . . . . . .4810116

Nephtys ferruginea ..............4810706

Oligochaete, unident. ............4880001

Pholoe glabra ...................44810442

Phoronis sp(p). ................5700002

Pseudopolydora paucibranchiata . . . . . 4810347

Schistomeringos rudolphi ...........4810354

Sphaerosyllis californiensis ........4810272

Spiophanes berkeleyorum ............4810465

Stylatula $\mathrm{sp}(\mathrm{p}) . \ldots \ldots \ldots \ldots \ldots . . . \ldots . . .3730044$

Tapes japonica ..................5540158

Tubulanus sp(p). .................4000013

Upogebia pugettensis ............5286103

$\begin{array}{rrrrr}1 & 2 & 2 & 2 & 1 \\ 1 & 0 & 9 & 6 & 0 \\ 0 & 0 & 0 & 1 & 0 \\ 0 & 0 & 0 & 0 & 1 \\ 0 & 0 & 3 & 0 & 0 \\ 0 & 0 & 2 & 2 & 3 \\ 0 & 0 & 0 & 1 & 0 \\ 0 & 0 & 1 & 0 & 0 \\ 0 & 0 & 0 & 0 & 1 \\ 0 & 0 & 11 & 6 & 2 \\ 0 & 3 & 32 & 29 & 8 \\ 0 & 0 & 0 & 0 & 2 \\ 0 & 0 & 3 & 0 & 0 \\ 0 & 0 & 3 & 0 & 0 \\ 0 & 0 & 3 & 0 & 0 \\ 0 & 8 & 10 & 7 & 0 \\ 0 & 0 & 1 & 0 & 0 \\ 1 & 6 & 2 & 8 & 3 \\ 0 & 2 & 1 & 0 & 0 \\ 1 & 2 & 1 & 1 & 0 \\ 0 & 2 & 0 & 10 & 1 \\ 0 & 0 & 0 & 0 & 1 \\ 0 & 0 & 1 & 0 & 0 \\ 0 & 0 & 1 & 0 & 0 \\ 0 & 0 & 1 & 2 & 0 \\ 1 & 0 & 1 & 0 & 0 \\ 0 & 1 & 0 & 0 & 0 \\ 0 & 9 & 2 & 9 & 0 \\ 0 & 14 & 20 & 78 & 1 \\ 1 & 0 & 0 & 1 & 3 \\ 2 & 0 & 0 & 0 & 0 \\ 0 & 11 & 5 & 17 & 1 \\ 0 & 0 & 0 & 1 & 0 \\ 0 & 16 & 1 & 0 & 182 \\ 1 & 1 & 0 & 2 & 0 \\ 0 & 1 & 1 & 0 & 3 \\ 0 & 9 & 5 & 30 & 3 \\ 0 & 0 & 1 & 0 & 0 \\ 0 & 1 & 1 & 4 & 1 \\ 1 & 0 & 1 & 0 & 0 \\ 0 & 0 & 1 & 0 & 0 \\ 0 & 0 & 2 & 0 & 0\end{array}$


Table 3.-Benthic macrofauna data-Continued

\begin{tabular}{|c|c|c|c|c|c|c|}
\hline \multirow{3}{*}{ Taxonomic entry } & \multirow{3}{*}{$\begin{array}{c}\text { Kinnetic } \\
\text { Laboratory } \\
\text { code }\end{array}$} & \multicolumn{5}{|c|}{ Individuals } \\
\hline & & \multicolumn{5}{|c|}{ Replicate number } \\
\hline & & 1 & 2 & 3 & 4 & 5 \\
\hline
\end{tabular}

\section{Berkeley}

Sampling date: May 31, 1988

\begin{tabular}{|c|c|c|c|c|c|}
\hline Amaeana occidentalis .............44810001 & 2 & 0 & 1 & 3 & 0 \\
\hline Ampelisca abdita ................5275504 & 12 & 7 & 6 & 5 & 15 \\
\hline$\ldots \ldots \ldots \ldots 4810565$ & 3 & 1 & 2 & 1 & 2 \\
\hline Capitella capitata ...............4810241 & 6 & 7 & 11 & 11 & 10 \\
\hline Cerebratulus $\mathrm{sp}(\mathrm{p}) . \ldots \ldots \ldots \ldots \ldots . . \ldots 4000014$ & 1 & 0 & 1 & 0 & 0 \\
\hline Cirriformia spirabrancha ........... 4810854 & 1 & 0 & 0 & 0 & 0 \\
\hline Corophium sp. a ..............5275510 & 229 & 115 & 28 & 349 & 179 \\
\hline Crangon $\mathrm{sp}(j u v) \ldots \ldots \ldots \ldots \ldots \ldots 5286127$ & 1 & 0 & 0 & 0 & 0 \\
\hline Cryptomya californica ............5540155 & 0 & 0 & 1 & 0 & 0 \\
\hline Dentinephtys sp. A (Harris) . . . . . . . . .4810904 & 0 & 1 & 2 & 0 & 0 \\
\hline Edwardsiidae, unident. ............3730045 & 1 & 2 & 2 & 1 & 1 \\
\hline Euchone limnicola ................4810255 & 2 & 0 & 0 & 0 & 4 \\
\hline Exogone lourei ................4810066 & 3 & 5 & 2 & 7 & 1 \\
\hline Glycinde polygnatha .............4810496 & 7 & 5 & 1 & 5 & 11 \\
\hline Harmothoe imbricata ..............4810343 & 0 & 0 & 0 & 1 & 0 \\
\hline Harmothoe lunulata ...............4810689 & 1 & 0 & 1 & 0 & 0 \\
\hline Hesperonoe $\mathrm{sp}(\mathrm{p}) . \ldots \ldots \ldots \ldots \ldots . . .4810090$ & 0 & 0 & 0 & 3 & 0 \\
\hline Leitoscoloplos pugettensis . . .......4810516 & 3 & 1 & 6 & 1 & 4 \\
\hline Leptochelia dubia .................55264038 & 1 & 0 & 0 & 1 & 0 \\
\hline Lightiella serendipita .............5204001 & 1 & 1 & 1 & 0 & 0 \\
\hline Macoma nasuta ..................5540019 & 1 & 0 & 0 & 0 & 0 \\
\hline Mysella sp. (A) (SCAMIT) . . . . . . . . . . . 5540212 & 5 & 1 & 2 & 24 & 1 \\
\hline Mytilus edulis .................5540024 & 1 & 0 & 0 & 0 & 0 \\
\hline Nematodes, unident. ..............4500001 & 3 & 0 & 2 & 5 & 0 \\
\hline Nephtys cornuta franciscana . ........4810116 & 8 & 2 & 8 & 2 & 5 \\
\hline Oligochaete, unident. .............4880001 & 16 & 10 & 35 & 16 & 12 \\
\hline Philine sp. (A) (SCAMIT) . . . . . . . . . 5570240 & 4 & 6 & 5 & 0 & 1 \\
\hline Phoronis $\mathrm{sp}(\mathrm{p}) . \ldots \ldots \ldots \ldots \ldots \ldots . \ldots . \ldots . \ldots 700002$ & 24 & 0 & 0 & 0 & 1 \\
\hline Potamocorbula amurensis . . . . . . . . 5540214 & 0 & 0 & 0 & 2 & 0 \\
\hline Pseudopolydora kempi .............4810640 & 0 & 0 & 0 & 2 & 0 \\
\hline ...........5220091 & 2 & 0 & 0 & 3 & 2 \\
\hline Schistomeringos rudolphi ..........4810354 & 0 & 0 & 0 & 0 & 2 \\
\hline Sphaerosyllis bilobata ..............4810833 & 1 & 0 & 0 & 0 & 0 \\
\hline Sphaerosyllis californiensis ..........4810272 & 18 & 3 & 8 & 8 & 8 \\
\hline 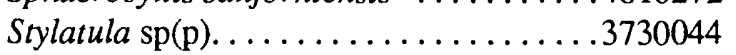 & 1 & 0 & 1 & 1 & 0 \\
\hline Tapes japonica ................... 5540158 & 1 & 0 & 0 & 0 & 0 \\
\hline Tenonia priops ................... 4810727 & 0 & 0 & 0 & 1 & 0 \\
\hline Tubulanus $\mathrm{sp}(\mathrm{p}) . \ldots \ldots \ldots \ldots \ldots \ldots . . .4000013$ & 0 & 1 & 1 & 0 & 0 \\
\hline Upogebia pugettensis .............5286103 & 0 & 3 & 0 & 0 & 0 \\
\hline
\end{tabular}


Table 3.-Benthic macrofauna data-Continued

\begin{tabular}{lcccccc}
\hline \multirow{2}{*}{ Taxonomic entry } & Kinnetic & \multicolumn{5}{c}{ Individuals } \\
\cline { 2 - 6 } & Laboratory & \multicolumn{4}{c}{ Replicate number } \\
\cline { 2 - 6 } & code & 1 & 2 & 3 & 4 & 5 \\
\hline
\end{tabular}

Berkeley

Sampling date: July 28,1988

\begin{tabular}{|c|c|c|c|c|}
\hline Amaeana occidentalis ........... & 0 & 0 & 1 & 0 \\
\hline Ampelisca abdita .............. & 8 & 3 & 2 & 6 \\
\hline Ampharete labrops ............. & 0 & 0. & 1 & 0 \\
\hline Asychis elongata ............... & 2 & 2 & 2 & 6 \\
\hline Campanularidae, unident. ............ 3710039 & 0 & 0 & 1 & 1 \\
\hline Capitella capitata ................4810241 & 13 & 2 & 3 & 0 \\
\hline Cerebratulus $\mathrm{sp}(\mathrm{p}) \ldots \ldots \ldots \ldots \ldots \ldots 4000014$ & 3 & 0 & 0 & 0 \\
\hline Corophium sp(p)..............5275098 & 2 & 2 & 1 & 2 \\
\hline Corophium sp. $a \ldots \ldots \ldots \ldots \ldots . \ldots 275510$ & 162 & 125 & 16 & 37 \\
\hline Cryptomya californica ............5540155 & 1 & 0 & 0 & 0 \\
\hline Edwardsia sipunculoides . . . . . . . . . 3730022 & 1 & 0 & 0 & 0 \\
\hline Edwardsiidae, unident. ..............3730045 & 0 & 2 & 1 & 1 \\
\hline Euchone limnicola ..............4810255 & 0 & 1 & 0 & 0 \\
\hline Glycinde polygnatha .............4810496 & 1 & 4 & 4 & 2 \\
\hline Leitoscoloplos pugettensis . ........4810516 & 1 & 3 & 1 & 1 \\
\hline Leptochelia dubia ...............55264038 & 1 & 0 & 0 & 0 \\
\hline Leucon subnasica ................55263012 & 0 & 0 & 1 & 0 \\
\hline Lightiella serendipita . . . . . . . . . 5204001 & 0 & 1 & 0 & 0 \\
\hline Lineidae, unident. ..............4000038 & 3 & 0 & 0 & 0 \\
\hline Musculista senhousia ...............5540401 & 1 & 0 & 0 & 0 \\
\hline Mya arenaria .................5540402 & 0 & 0 & 0 & 1 \\
\hline Mysella sp. (A) (SCAMIT) . . . . . . . . . .5540212 & 0 & 0 & 2 & 0 \\
\hline 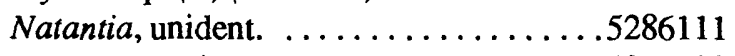 & 1 & 1 & 0 & 0 \\
\hline Nemertea, unident. . ...........4000002 & 0 & 0 & 0 & 2 \\
\hline Nephtys cornuta franciscana . . . . . . . .4810116 & 2 & 1 & 2 & 1 \\
\hline Ostracods, unident. .............5220003 & 0 & 1 & 0 & 0 \\
\hline Phoronis $\mathrm{sp}(\mathrm{p}) . \ldots \ldots \ldots \ldots \ldots \ldots . .5700002$ & 189 & 1 & 14 & 59 \\
\hline Photis brevipes ................5275109 & 0 & 0 & 0 & 4 \\
\hline Pleusymtes sp(p). ...............5275203 & 0 & 1 & 1 & 0 \\
\hline Potamocorbula amurensis . .........5540214 & 0 & 1 & 0 & 0 \\
\hline Protothaca staminea ..............5540035 & 0 & 0 & 1 & 1 \\
\hline Pseudopolydorakempi ............4810640 & 1 & 0 & 1 & 0 \\
\hline Pyromaia tuberculata ..............5286094 & 0 & 0 & 1 & 0 \\
\hline Sarsiella zostericola .............5220091 & 0 & 0 & 0 & 0 \\
\hline Schistomeringos rudolphi ..........4810354 & 2 & 1 & 1 & 0 \\
\hline Scleroplax granulata ..............5286519 & 0 & 0 & 0 & 0 \\
\hline Scolelepis squamata ..............4810589 & 0 & 1 & 0 & 0 \\
\hline Spiophanes berkeleyorum ...........4810465 & 0 & 1 & 0 & 0 \\
\hline 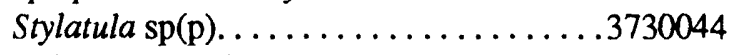 & 3 & 1 & 0 & 1 \\
\hline Tubulanus $\mathrm{sp}(\mathrm{p}) . \ldots \ldots \ldots \ldots \ldots . \ldots 4000013$ & 0 & 1 & 0 & 0 \\
\hline Upogebia pugettensis ...........5286103 & 1 & 0 & 0 & 0 \\
\hline
\end{tabular}


Table 3.-Benthic macrofauna data-Continued

\begin{tabular}{lccccc}
\hline \multirow{2}{*}{ Taxonomic entry } & Kinnetic & \multicolumn{5}{c}{ Individuals } \\
\cline { 3 - 6 } & Laboratory & \multicolumn{4}{c}{ Replicate number } \\
\cline { 3 - 6 } & code & 1 & 2 & 3 & 4 \\
\hline
\end{tabular}

\section{Berkeley}

Sampling date: September 13, 1988

\begin{tabular}{|c|c|c|c|c|c|}
\hline Amaeana occidentalis & 0 & 1 & 0 & 1 & 0 \\
\hline Ampelisca abdita & 2 & 7 & 5 & 1 & 1 \\
\hline Asychis elongata & 4 & 1 & 0 & 1 & 1 \\
\hline Campanularidae, unident. ...........3710039 & 1 & 0 & 0 & 0 & 0 \\
\hline Capitella capitata ...............4810241 & 0 & 0 & 3 & 2 & 3 \\
\hline Cerebratulus $\mathrm{sp}(\mathrm{p}) . \ldots \ldots \ldots \ldots \ldots . \ldots 4000014$ & 1 & 0 & 1 & 1 & 1 \\
\hline Cooperella subdiaphana ............5540056 & 0 & 0 & 0 & 0 & 1 \\
\hline Corophium $\mathrm{sp}(\mathrm{p})$................5275098 & 0 & 0 & 0 & 0 & 1 \\
\hline Corophium sp. a .............5275510 & 77 & 50 & 53 & 11 & 82 \\
\hline Cryptomya californica ............5540155 & 8 & 10 & 6 & 0 & 4 \\
\hline Diopatra $\mathrm{sp}(\mathrm{p}) . \ldots \ldots \ldots \ldots \ldots \ldots . \ldots 4810999$ & 0 & 1 & 0 & 0 & 0 \\
\hline Edwardsia $\mathrm{sp}(\mathrm{p}) \ldots \ldots \ldots \ldots \ldots \ldots . \ldots 3730037$ & 0 & 0 & 1 & 0 & 0 \\
\hline Edwardsiidae, unident. ............3730045 & 1 & 1 & 1 & 0 & 0 \\
\hline Euchone limnicola ..............4810255 & 0 & 0 & 0 & 0 & 1 \\
\hline Exogone lourei ...............4810066 & 0 & 0 & 1 & 0 & 1 \\
\hline Glycinde polygnatha ............44810496 & 4 & 8 & 6 & 4 & 6 \\
\hline Harmothoe imbricata .............4810343 & 0 & 0 & 0 & 0 & 1 \\
\hline Harpacticoid copepod, unident. ........5230052 & 1 & 0 & 1 & 0 & 0 \\
\hline Heteromastus filiformis . . . . . . . . . 4810438 & 1 & 0 & 0 & 0 & 0 \\
\hline Hydrozoa, unident. . .............3710052 & 1 & 0 & 0 & 0 & 0 \\
\hline Leitoscoloplos pugettensis $\ldots \ldots \ldots \ldots 4810516$ & 0 & 7 & 4 & 3 & 2 \\
\hline Leptochelia dubia ...............5264038 & 0 & 0 & 0 & 0 & 1 \\
\hline Leucon subnasica ...............5263012 & 0 & 1 & 0 & 0 & 0 \\
\hline Lineidae, unident. . ............4000038 & 0 & 0 & 2 & 0 & 1 \\
\hline Mysella sp. (A) (SCAMIT) . ...........5540212 & 0 & 1 & 0 & 0 & 0 \\
\hline Nematodes, unident. . ............4500001 & 0 & 0 & 0 & 0 & 1 \\
\hline Nephtys caecoides ...............44810114 & 0 & 0 & 0 & 0 & 1 \\
\hline Nephtys cornuta franciscana .........4810116 & 3 & 2 & 2 & 8 & 1 \\
\hline Oligochaete, unident. . ............4880001 & 1 & 7 & 1 & 10 & 1 \\
\hline Pennatulacea, unident. ...........3700002 & 0 & 1 & 1 & 0 & 2 \\
\hline Philine sp. (A) (SCAMIT) . . . . . . . . . 5570240 & 1 & 0 & 1 & 3 & 3 \\
\hline Phoronis $\mathrm{sp}(\mathrm{p}) . \ldots \ldots \ldots \ldots \ldots . \ldots 5700002$ & 13 & 120 & 330 & 35 & 352 \\
\hline Photis brevipes ...............5275109 & 0 & 0 & 3 & 0 & 1 \\
\hline Platyhelminthid, unident. ...........3900001 & 0 & 1 & 0 & 0 & 0 \\
\hline Potamocorbula amurensis ..........5540214 & 0 & 0 & 3 & 5 & 1 \\
\hline Protothaca staminea ..............5540035 & 1 & 1 & 1 & 1 & 1 \\
\hline Pseudopolydora kempi ............4810640 & 0 & 0 & 0 & 1 & 0 \\
\hline Pseudopolydora paucibranchiata ......4810347 & 0 & 0 & 0 & 2 & 0 \\
\hline Sarsiella zostericola .............55220091 & 0 & 0 & 2 & 1 & 0 \\
\hline Solen sicarius .................5540416 & 0 & 0 & 0 & 1 & 1 \\
\hline Spiophanes missionensis . . . . . . . . . .4810204 & 1 & 0 & 0 & 0 & 0 \\
\hline Tenonia priops . ................4810727 & 0 & 0 & 1 & 0 & 0 \\
\hline
\end{tabular}


Table 3.-Benthic macrofauna data-Continued

\begin{tabular}{lccccc}
\hline \multirow{2}{*}{ Taxonomic entry } & Kinnetic & \multicolumn{5}{c}{ Individuals } \\
\cline { 2 - 6 } & Laboratory & \multicolumn{4}{c}{ Replicate number } \\
\cline { 2 - 6 } & code & 1 & 2 & 3 & 4 \\
\hline
\end{tabular}

Berkeley

Sampling date: September 13, 1988-Continued

Theora lubrica ....................55540114

Tubulanus sp(p). ...............4000013

Upogebia pugettensis

.5286103

$\begin{array}{ll}0 & 0 \\ 3 & 0 \\ 1 & 0\end{array}$

0
1
0

0

0

Sampling date: November 1, 1988

Amaeana occidentalis ............44810001

Ampelisca abdita ................ 5275504

Asychis elongata .............. 4810565

Campanularidae, unident. . . . . . . . . . 3710039

Capitella capitata ...............4810241

Cerebratulus sp(p). ...............4000014

Clinocardium nuttallii . . . . . . . . . . . . 5540417

Corophium sp. a ................5275510

Cryptomya californica ............55540155

Edwardsia sipunculoides ...........3730022

Edwardsiidae, unident. ..............3730045

Euchone limnicola ................ 4810255

Eusyllis transecta ...............44810680

Glycinde polygnatha ..............44810496

Leitoscoloplos pugettensis ..........4810516

Leptochelia dubia .................55264038

Leucon subnasica ................5263012

Lightiella serendipita .............5204001

Macoma indentata ...............5540176

Mactra sp(p). .................5540122

Melanochlamys diomedea ...........5570248

Molgula manhattensis .............6301075

Musculista senhousia ..............5540401

Neanthes virens . ...............4810560

Nematodes, unident. .............4500001

Nephtys caecoides ................4810114

Nephtys cornuta franciscana . . . . . . . . 4810116

Nephtys sp(p). .................4810579

Oligochaete, unident. . ............4880001

Philine sp. (A) (SCAMIT) . . . . . . . . 5570240

Phoronis sp(p). . ..............55700002

Photis sp(p).....................55275013

Polydora socialis ................4810940

$\begin{array}{rrrrr}0 & 0 & 0 & 1 & 0 \\ 10 & 10 & 2 & 0 & 26 \\ 5 & 0 & 2 & 3 & 3 \\ 1 & 0 & 0 & 1 & 0 \\ 1 & 3 & 4 & 0 & 3 \\ 0 & 1 & 0 & 0 & 0 \\ 0 & 0 & 0 & 1 & 0 \\ 55 & 22 & 22 & 0 & 4 \\ 30 & 26 & 0 & 0 & 11 \\ 0 & 0 & 0 & 0 & 1 \\ 0 & 1 & 2 & 0 & 1 \\ 0 & 0 & 0 & 3 & 0 \\ 0 & 1 & 0 & 0 & 0 \\ 4 & 6 & 4 & 6 & 5 \\ 1 & 1 & 2 & 1 & 1 \\ 0 & 1 & 0 & 0 & 0 \\ 1 & 0 & 0 & 0 & 0 \\ 0 & 1 & 0 & 0 & 0 \\ 0 & 1 & 0 & 0 & 0 \\ 0 & 0 & 0 & 1 & 0 \\ 0 & 1 & 0 & 0 & 0 \\ 0 & 1 & 0 & 0 & 0 \\ 1 & 0 & 0 & 0 & 0 \\ 0 & 1 & 0 & 0 & 0 \\ 0 & 0 & 0 & 4 & 0 \\ 1 & 0 & 0 & 0 & 1 \\ 0 & 1 & 0 & 3 & 2 \\ 0 & 0 & 2 & 0 & 0 \\ 7 & 2 & 0 & 1 & 0 \\ 1 & 1 & 2 & 0 & 2 \\ 58 & 580 & 41 & 1 & 59 \\ 0 & 4 & 2 & 0 & 3 \\ 0 & 1 & 0 & 0 & 0\end{array}$


Table 3.-Benthic macrofauna data-Continued

\begin{tabular}{lccccc}
\hline \multirow{2}{*}{ Taxonomic entry } & Kinnetic & \multicolumn{5}{c}{ Individuals } \\
\cline { 3 - 6 } & Laboratory & \multicolumn{4}{c}{ Replicate number } \\
\cline { 2 - 6 } & code & 1 & 2 & 3 & 4 \\
\hline
\end{tabular}

$\underline{\text { Berkeley }}$

Sampling date: November 1, 1988 -Continued

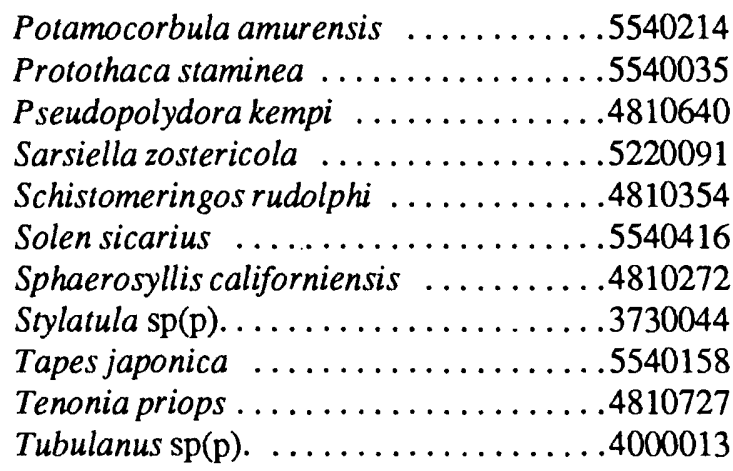

$\begin{array}{ll}1 & 0 \\ 0 & 0 \\ 0 & 0 \\ 1 & 1 \\ 1 & 1 \\ 1 & 0 \\ 0 & 0 \\ 1 & 1 \\ 0 & 1 \\ 0 & 1 \\ 2 & 0\end{array}$

Coyote Point

Sampling date: January 13,1988

\begin{tabular}{|c|c|c|c|c|c|}
\hline Amaeana occidentalis .............4810001 & 0 & 0 & 1 & 0 & 0 \\
\hline Ampelisca abdita ................5275504 & 269 & 215 & 189 & 139 & 138 \\
\hline Anaitides longipes ..............4810505 & 1 & 0 & 0 & 0 & 0 \\
\hline Anthozoan, unident. . . . . . . . . . 3730010 & 0 & 0 & 1 & 0 & 0 \\
\hline Asychis elongata ................4810565 & 27 & 39 & 22 & 20 & 25 \\
\hline Capitella capitata ..............4810241 & 0 & 0 & 0 & 0 & 1 \\
\hline Corophium alienense ................5275287 & 12 & 15 & 29 & 19 & 20 \\
\hline Eusyllis transecta ..............4810680 & 15 & 9 & 4 & 7 & 12 \\
\hline Exogone lourei ...............44810066 & 1 & 3 & 3 & 3 & 0 \\
\hline Gemma gemma ..................5540400 & 0 & 0 & 1 & 0 & 0 \\
\hline Glycinde polygnatha ..............4810496 & 9 & 11 & 10 & 5 & 6 \\
\hline Heteromastus filiformis . . . . . . . . . . . 4810438 & 0 & 0 & 1 & 0 & 0 \\
\hline Leitoscoloplos pugettensis ...........4810516 & 2 & 0 & 0 & 0 & 1 \\
\hline Macoma balthica ..................5540147 & 0 & 0 & 0 & 1 & 0 \\
\hline Mediomastussp. ................4810303 & 1 & 1 & 0 & 0 & 0 \\
\hline Musculista senhousia ...............5540401 & 7 & 3 & 3 & 2 & 6 \\
\hline Mya arenaria ...................5540402 & 0 & 0 & 0 & 0 & 2 \\
\hline Nematodes, unident. . ..............4500001 & 2 & 10 & 12 & 11 & 0 \\
\hline Nephtys caecoides ................4810114 & 1 & 1 & 1 & 0 & 0 \\
\hline Nephtys cornuta franciscana . . . . . . . 4810116 & 1 & 1 & 2 & 0 & 0 \\
\hline Odostomia (Evalea) sp. $N$ (Shrake) $\ldots . .5570321$ & 0 & 1 & 0 & 0 & 1 \\
\hline Oligochaete, unident. . . . . . . . . . . .4880001 & 20 & 63 & 47 & 53 & 7 \\
\hline Philine sp. (A) (SCAMIT) . . . . . . . . . 5570240 & 0 & 1 & 0 & 0 & 0 \\
\hline Potamocorbula amurensis ..........5540214 & 4 & 4 & 4 & 3 & 2 \\
\hline Pseudopolydora kempi .............4810640 & 1 & 1 & 6 & 1 & 3 \\
\hline Sarsiella zostericola ...............55220091 & 12 & 5 & 7 & 5 & 12 \\
\hline
\end{tabular}


Table 3.-Benthic macrofauna data-Continued

\begin{tabular}{|c|c|c|c|c|c|c|}
\hline \multirow{3}{*}{ Taxonomic entry } & \multirow{3}{*}{$\begin{array}{c}\text { Kinnetic } \\
\text { Laboratory } \\
\text { code }\end{array}$} & \multicolumn{5}{|c|}{ Individuals } \\
\hline & & \multicolumn{5}{|c|}{ Replicate number } \\
\hline & & 1 & 2 & 3 & 4 & $\overline{5}$ \\
\hline
\end{tabular}

Coyote Point

Sampling date: January $13,1988-$ Continued

Schistomeringos rudolphi ..........4810354

Streblospio benedicti ..............4810257

Tapes japonica .................5540158

Theora lubrica ...................5540114

$\begin{array}{rr}0 & 1 \\ 0 & 0 \\ 35 & 40 \\ 7 & 4\end{array}$

Sampling date: March 8, 1988

Ampelisca abdita ................5275504

Asychis elongata ................4810565

Campanularidae, unident. ...........3710039

Corophium alienense . . . . . . . . . . . . 5275287

Corophium sp(p). . . . . . . . . . . . . 5275098

Euchone limnicola ...............4810255

Exogone lourei ...............4810066

Gemma gemma ..................5540400

Glycera americana ................4810487

Glycinde polygnatha ..............4810496

Heteromastus filiformis . . . . . . . . . . 4810438

Leitoscoloplos pugettensis $\ldots \ldots \ldots \ldots .4810516$

Leucon subnasica ................... 5263012

Musculista senhousia ...............5540401

Neanthes succinea . . . . . . . . . . . . . . 4810562

Nematodes, unident. ..............4500001

Nephtys caecoides ................4810114

Nephtys cornuta franciscana .........4810116

Odostomia (Evalea) sp. I (Shrake) . . ...55570317

Oligochaete, unident. .............4880001

Polydora brachycephala ............4810557

Polynoid, unident. ................4810936

Potamocorbula amurensis . . . . . . . . 5540214

Pseudopolydora kempi ............4810640

Sarsiella zostericola ............. 5220091

Schistomeringos rudolphi ..........4810354

Sphaerosyllis californiensis . ........4810272

Streblospio benedicti ...............4810257

Tapes japonica .................55540158

Theora lubrica ...................55540114

Upogebia pugettensis ...........5286103

$\begin{array}{rrrr}46 & 34 & 142 & 54 \\ 21 & 28 & 34 & 32 \\ 0 & 1 & 1 & 0 \\ 11 & 1 & 11 & 6 \\ 6 & 0 & 0 & 5 \\ 4 & 2 & 2 & 0 \\ 20 & 8 & 5 & 5 \\ 3 & 1 & 0 & 0 \\ 0 & 0 & 0 & 1 \\ 6 & 3 & 9 & 3 \\ 1 & 0 & 2 & 0 \\ 0 & 1 & 0 & 0 \\ 0 & 1 & 0 & 0 \\ 11 & 13 & 29 & 5 \\ 0 & 1 & 0 & 0 \\ 0 & 12 & 6 & 2 \\ 1 & 2 & 0 & 0 \\ 1 & 0 & 0 & 0 \\ 0 & 0 & 0 & 1 \\ 40 & 43 & 2 & 1 \\ 3 & 3 & 2 & 0 \\ 1 & 0 & 0 & 0 \\ 4 & 2 & 3 & 2 \\ 2 & 0 & 0 & 0 \\ 2 & 1 & 3 & 2 \\ 1 & 0 & 1 & 0 \\ 0 & 1 & 0 & 0 \\ 1 & 0 & 0 & 1 \\ 33 & 26 & 43 & 4 \\ 3 & 2 & 0 & 0 \\ 1 & 0 & & 0\end{array}$


Table 3.-Benthic macrofauna data-Continued

\begin{tabular}{lccccc}
\hline \multirow{2}{*}{ Taxonomic entry } & Kinnetic & \multicolumn{5}{c}{ Individuals } \\
\cline { 2 - 6 } & Laboratory & \multicolumn{4}{c}{ Replicate number } \\
\cline { 2 - 6 } & code & 1 & 2 & 3 & 4 \\
\hline
\end{tabular}

\section{Coyote Point}

Sampling date: May 31, 1988

\begin{tabular}{|c|c|c|c|c|c|}
\hline Amaeana occidentalis .............4810001 & 0 & 0 & 0 & 1 & 0 \\
\hline Ampelisca abdita & 121 & 118 & 91 & 175 & 89 \\
\hline Amphiurid, unident. . . . . . . . . . . . 5930014 & 1 & 0 & 0 & 0 & 0 \\
\hline Anaitides longipes . . . . . . . . . . 4810505 & 1 & 3 & 0 & 0 & 0 \\
\hline Anthozoan, unident. . . . . . . . . . . 3730010 & 1 & 0 & 0 & 1 & 0 \\
\hline Asychis elongata ................4810565 & 43 & 28 & 12 & 40 & 37 \\
\hline Balanus crenatus . ...............5250036 & 1 & 0 & 0 & 0 & 0 \\
\hline Brachyuran megalops ..............5286064 & 1 & 0 & 0 & 0 & 0 \\
\hline Capitella capitata ...............4810241 & 0 & 1 & 0 & 3 & 2 \\
\hline Corophium $\mathrm{sp}(\mathrm{p}) \ldots \ldots \ldots \ldots \ldots \ldots . \ldots 5275098$ & 0 & 3 & 1 & 0 & 1 \\
\hline Corophium sp. $a \ldots \ldots \ldots \ldots \ldots . \ldots 5275510$ & 371 & 325 & 391 & 492 & 404 \\
\hline Cryptomya californica ............5540155 & 0 & 0 & 0 & 0 & 1 \\
\hline Euchone limnicola ...............4810255 & 5 & 4 & 5 & 7 & 5 \\
\hline Exogone lourei $\ldots \ldots \ldots \ldots \ldots \ldots 4810066$ & 5 & 13 & 17 & 8 & 17 \\
\hline Gemma gemma ................55540400 & 0 & 0 & 1 & 0 & 0 \\
\hline Glycinde polygnatha .............4810496 & 19 & 28 & 24 & 27 & 40 \\
\hline Harmothoe imbricata .............4810343 & 2 & 0 & 3 & 1 & 1 \\
\hline Leitoscoloplos pugettensis . .........4810516 & 0 & 0 & 0 & 0 & 2 \\
\hline Leptochelia dubia ..............55264038 & 0 & 0 & 0 & 2 & 1 \\
\hline Leucon subnasica ..............55263012 & 0 & 1 & 1 & 1 & 0 \\
\hline Lightiella serendipita . . . . . . . . . . 5204001 & 0 & 1 & 0 & 0 & 0 \\
\hline Musculista senhousia .............5540401 & 52 & 40 & 31 & 29 & 32 \\
\hline Nematodes, unident. .............4500001 & 0 & 0 & 0 & 2 & 0 \\
\hline Nephtys caecoides . .............. 4810114 & 0 & 0 & 1 & 0 & 1 \\
\hline Nephtys cornuta franciscana . .........4810116 & 0 & 2 & 5 & 4 & 1 \\
\hline Oligochaete, unident. ............4880001 & 1 & 0 & 7 & 6 & 3 \\
\hline Philine sp. (A) (SCAMIT) . . . . . . . . . 55570240 & 0 & 0 & 2 & 2 & 2 \\
\hline Polydora brachycephala ............4810557 & 4 & 1 & 3 & 0 & 4 \\
\hline Potamocorbula amurensis . .........5540214 & 1 & 4 & 5 & 3 & 1 \\
\hline Pseudopolydora paucibranchiata . . .... 4810347 & 0 & 0 & 0 & 3 & 0 \\
\hline Pyromaia tuberculata . . . . . . . . . . . . 5286094 & 0 & 0 & 0 & 0 & 1 \\
\hline Sarsiella zostericola ..............5220091 & 0 & 0 & 5 & 6 & 3 \\
\hline Schistomeringos rudolphi ...........4810354 & 3 & 0 & 7 & 1 & 0 \\
\hline Siliqua lucida ...................55540043 & 0 & 0 & 0 & 0 & 1 \\
\hline Sphaerosyllis californiensis . . .......4810272 & 1 & 0 & 1 & 0 & 1 \\
\hline Tapes japonica . . . . . . . . . . . . . . . . .5540158 & 46 & 41 & 42 & 18 & 32 \\
\hline Theora lubrica ..................... .5540114 & 1 & 2 & 3 & 3 & 0 \\
\hline Upogebia pugettensis ............5286103 & 0 & 0 & 1 & 0 & 1 \\
\hline
\end{tabular}


Table 3.-Benthic macrofauna data-Continued

\begin{tabular}{|c|c|c|c|c|c|}
\hline \multirow{3}{*}{ Taxonomic entry } & \multirow{3}{*}{$\begin{array}{c}\text { Kinnetic } \\
\text { Laboratory } \\
\text { code }\end{array}$} & \multicolumn{4}{|c|}{ Individuals } \\
\hline & & \multicolumn{4}{|c|}{ Replicate number } \\
\hline & & 1 & 3 & 4 & 5 \\
\hline
\end{tabular}

Coyote Point

Sampling date: July 25,1988

Ampelisca abdita .................. 5275504

Anthozoan, unident. ................. 3730010

Asychis elongata ..............4810565

Campanularidae, unident. ............3710039

Capitella capitata ................ 4810241

Cheilostomata unident. ..............5600177

Corophium sp(p)...................55275098

Corophium sp. a ................55275510

Euchone limnicola ................4810255

Exogone lourei ................4810066

Glycinde polygnatha .............4810496

Harmothoe imbricata . . . . . . . . . . . 4810343

Heteromastus filiformis . . . . . . . . . . .4810438

Hydrozoa, unident. ................3710052

Leitoscoloplos pugettensis . . . . . . . .4810516

Leptochelia dubia ................5264038

Marphysa sanguinea . . . . . . . . . . . 4810248

Mediomastus sp. ................4810303

Molgula manhattensis . . . . . . . . . .6301075

Musculista senhousia . . . . . . . . . . . .5540401

Nephtys caecoides .................4810114

Nephtys cornuta franciscana . . . . . . . . .4810116

Oligochaete, unident. ............4880001

Philine sp. (A) (SCAMIT) ...........5570240

Polydora ligni ..................4810168

Potamocorbula amurensis .......... 5540214

Pseudopolydora paucibranchiata ...... 4810347

Sarsiella zostericola ..............5220091

Schistomeringos rudolphi ............4810354

Streblospio benedicti ................4810257

Tapes japonica .................55540158

Theora lubrica . . . . . . . . . . . . . . . . 5540114
Phoronis $\mathrm{sp}(\mathrm{p}) . \ldots \ldots \ldots \ldots \ldots . \ldots . \ldots . \ldots 0002$

$\begin{array}{rrrrr}8 & 15 & 14 & 14 & 6 \\ 0 & 2 & 0 & 0 & 1 \\ 53 & 68 & 25 & 39 & 25 \\ 0 & 1 & 1 & 1 & 0 \\ 1 & 0 & 0 & 1 & 0 \\ 0 & 1 & 1 & 1 & 0 \\ 0 & 1 & 3 & 3 & 0 \\ 16 & 12 & 32 & 19 & 6 \\ 7 & 31 & 20 & 21 & 3 \\ 1 & 2 & 2 & 15 & 0 \\ 2 & 1 & 2 & 3 & 0 \\ 2 & 2 & 1 & 4 & 2 \\ 0 & 2 & 0 & 0 & 0 \\ 0 & 0 & 1 & 1 & 1 \\ 0 & 1 & 2 & 4 & 2 \\ 1 & 0 & 0 & 1 & 0 \\ 0 & 0 & 0 & 1 & 0 \\ 1 & 0 & 0 & 0 & 0 \\ 0 & 0 & 1 & 1 & 0 \\ 10 & 10 & 25 & 16 & 22 \\ 1 & 0 & 0 & 0 & 0 \\ 1 & 1 & 0 & 1 & 0 \\ 0 & 1 & 0 & 2 & 0 \\ 2 & 0 & 1 & 0 & 2 \\ 0 & 0 & 0 & 0 & 9 \\ 0 & 2 & 2 & 2 & 0 \\ 2 & 4 & 0 & 3 & 0 \\ 0 & 1 & 1 & 0 & 1 \\ 0 & 5 & 2 & 1 & 0 \\ 0 & 0 & 0 & 1 & 0 \\ 0 & 0 & 0 & 1 & 0 \\ 6 & 16 & 9 & 9 & 5 \\ 4 & 10 & 6 & 8 & 0\end{array}$

Sampling date: September 14, 1988

Ampelisca abdita ...............55275504

Amphiuridae, unident. .............5930032

Anthozoan, unident. . ............. 3730010

Asychis elongata ................4810565

Campanularidae, unident. . . . . . . . . . .3710039

Capitella capitata

4810241

$\begin{array}{rrr}93 & 112 & 106 \\ 0 & 0 & 0 \\ 0 & 1 & 1 \\ 82 & 27 & 31 \\ 0 & 0 & 0 \\ 0 & 2 & 0\end{array}$


Table 3.-Benthic macrofauna data-Continued

\begin{tabular}{lccccc}
\hline \multirow{2}{*}{ Taxonomic entry } & Kinnetic & \multicolumn{5}{c}{ Individuals } \\
\cline { 2 - 6 } & Laboratory & \multicolumn{4}{c}{ Replicate number } \\
\cline { 2 - 6 } & code & 1 & 2 & 3 & 4 \\
\hline
\end{tabular}

$\underline{\text { Coyote Point }}$

Sampling date: September 14, 1988-Continued

\begin{tabular}{|c|c|c|c|c|c|}
\hline Cirratulidae, unident. . ............4810990 & 1 & 3 & 1 & 3 & 2 \\
\hline Corophium $\mathrm{sp}(\mathrm{p}) \ldots \ldots \ldots \ldots \ldots \ldots . \ldots 5275098$ & 1 & 0 & 0 & 0 & 0 \\
\hline Corophium sp. $a$ & 19 & 7 & 4 & 7 & 18 \\
\hline Crepidula plana ...................5570204 & 0 & 0 & 1 & 0 & 0 \\
\hline Crepidula $\mathrm{sp}(\mathrm{p}) \ldots . . \ldots \ldots \ldots . \ldots . \ldots . . .5570203$ & 1 & 0 & 0 & 0 & 0 \\
\hline Crepidula sp. A (Shrake) . . . . . . . . & 0 & 0 & 1 & 0 & 0 \\
\hline Cryptomya californica ............5540155 & 0 & 0 & 0 & 1 & 0 \\
\hline Dorvilleidae, unident. . . . . . . . . . . . .4810583 & 1 & 3 & 7 & 2 & 0 \\
\hline Euchone limnicola ................. 4810255 & 125 & 137 & 242 & 192 & 132 \\
\hline Eudorella pacifica ...............5263112 & 0 & 0 & 0 & 1 & 0 \\
\hline Eusyllis transecta ...............4810680 & 0 & 3 & 0 & 0 & 0 \\
\hline Exogone lourei ..................4810066 & 196 & 238 & 315 & 278 & 181 \\
\hline Gemma gemma .................5540400 & 1 & 0 & 0 & 0 & 0 \\
\hline Glycinde polygnatha . . . . . . . . . . . 44810496 & 0 & 3 & 1 & 1 & 5 \\
\hline Grandidierella japonica ............5275503 & 12 & 3 & 4 & 2 & 4 \\
\hline Harmothoe imbricata ...............4810343 & 2 & 4 & 3 & 2 & 1 \\
\hline Harmothoe lunulata ..............4810689 & 0 & 1 & 0 & 1 & 0 \\
\hline Leitoscoloplos pugettensis .........4810516 & 1 & 1 & 0 & 2 & 0 \\
\hline Leptochelia dubia ...............5264038 & 3 & 0 & 3 & 2 & 0 \\
\hline Leucon subnasica ................5263012 & 0 & 0 & 0 & 1 & 1 \\
\hline Macoma $\operatorname{sp(p).\ldots \ldots \ldots \ldots \ldots \ldots \ldots ...5540105}$ & 0 & 0 & 0 & 0 & 1 \\
\hline Melanochlamys diomedea ............5570248 & 0 & 1 & 0 & 0 & 0 \\
\hline Molgula manhattensis . . . . . . . . . . . 6301075 & 0 & 35 & 6 & 8 & 1 \\
\hline Musculista senhousia ............5540401 & 48 & 60 & 52 & 54 & 16 \\
\hline Natantia, unident. . ............5286111 & 0 & 0 & 1 & 0 & 0 \\
\hline Neanthes succinea . . ............. .4810562 & 1 & 0 & 0 & 2 & 0 \\
\hline Nematodes, unident. ..............4500001 & 93 & 7 & 13 & 61 & 1 \\
\hline Nephtys cornuta franciscana . . . . . . . . . 4810116 & 2 & 4 & 1 & 2 & 1 \\
\hline Nudibranchia, unident. ..............5570976 & 0 & 0 & 1 & 0 & 0 \\
\hline Odostomia (Evalea) sp. H (Shrake) .....5570314 & 1 & 1 & 1 & 1 & 0 \\
\hline Oligochaete, unident. ..............4880001 & 38 & 32 & 71 & 39 & 44 \\
\hline Philine sp. (A) (SCAMIT) ..........5570240 & 0 & 0 & 2 & 2 & 0 \\
\hline Phoronis $\mathrm{sp}(\mathrm{p}) . \ldots \ldots \ldots \ldots \ldots \ldots . \ldots 5700002$ & 1 & 0 & 2 & 10 & 1 \\
\hline Platyhelminthid, unident. . . . . . . . . 3900001 & 0 & 0 & 0 & 0 & 1 \\
\hline Polydora brachycephala ............4810557 & 3 & 5 & 4 & 5 & 7 \\
\hline Polydora ligni ..................4810168 & 92 & 115 & 166 & 123 & 174 \\
\hline Potamocorbula amurensis ...........5540214 & 40 & 65 & 21 & 50 & 52 \\
\hline Pseudopolydora kempi .............4810640 & 0 & 6 & 13 & 8 & 12 \\
\hline Pseudopolydora paucibranchiata ........4810347 & 59 & 57 & 62 & 54 & 38 \\
\hline Pyromaia tuberculata .............. 5286094 & 0 & 0 & 4 & 1 & 0 \\
\hline Sarsiella zostericola ...............5220091 & 13 & 8 & 11 & 12 & 10 \\
\hline Schistomeringos rudolphi ...........4810354 & 2 & 0 & 3 & 4 & 1 \\
\hline Sphaerosyllis bilobata.............4810833 & 0 & 3 & 2 & 5 & 1 \\
\hline
\end{tabular}


Table 3.-Benthic macrofauna data-Continued

\begin{tabular}{lcccccc}
\hline \multirow{3}{*}{ Taxonomic entry } & Kinnetic & \multicolumn{5}{c}{ Individuals } \\
\cline { 2 - 6 } & Laboratory & \multicolumn{4}{c}{ Replicate number } \\
\cline { 2 - 6 } & code & 1 & 2 & 3 & 4 & 5 \\
\hline
\end{tabular}

Coyote Point

Sampling date: September 14, 1988-Continued

Sphaerosyllis californiensis . . . . . . .4810272

Streblospio benedicti ..............4810257

Tapes japonica .................5540158

Tenonia priops . . . . . . . . . . . . . 4810727

Theora lubrica ................... 5540114

Tubulanus pellucidus .

4000011

$\begin{array}{rr}5 & 17 \\ 15 & 28 \\ 39 & 43 \\ 2 & 0 \\ 31 & 23 \\ 1 & 0\end{array}$

18
16
32
0
12
0

10

11

17

30

43

0

32

1

38

0

0

Sampling date: November 2, 1988

Amaeana occidentalis ..............4810001

Ampelisca abdita .................55275504

Amphiodia sp(p). .................5930040

Amphiuridae, unident. .............. 5930032

Anthozoan, unident. . . . . . . . . . . 3730010

Asychis elongata ...............4810565

Brachyuran megalops .............5286064

Brachyuran zoea .................. 5286063

Campanularidae, unident. ............3710039

Capitella capitata ...............4810241

Caprella sp(p)..................55275117

Cirratulidae, unident. . . . . . . . . . . .4810990

Corophium sp(p)..................5275098

Corophium sp. a ...............5275510

Cossura pygodactylata ...........4810861

Crangon nigricauda .............55286120

Crepidula plana .................5570204

Crepidula sp(p)....................55570203

Crepidula sp. A (Shrake) ............55570331

Diopatra sp(p). .................4810999

Edwardsiidae, unident. . . . . . . . . . 3730045

Euchone limnicola ...............4810255

Exogone lourei ................4810066

Gemma gemma .................55540400

Glycera americana ...............4810487

Glycinde polygnatha ...............4810496

Grandidierella japonica ............5275503

Harmothoe imbricata ..............4810343

Leitoscoloplos pugettensis .........4810516

Leptochelia dubia ................5264038

Macoma acolasta ...............55540026

Macoma $\mathrm{sp}(\mathrm{p}) . \ldots \ldots \ldots \ldots \ldots \ldots . \ldots 5540105$

$\begin{array}{rr}0 & 0 \\ 1,512 & 1,013 \\ 0 & 0 \\ 0 & 1 \\ 2 & 1 \\ 20 & 22 \\ 0 & 2 \\ 1 & 0 \\ 0 & 1 \\ 1 & 0 \\ 0 & 0 \\ 2 & 4 \\ 1 & 0 \\ 46 & 12 \\ 4 & 3 \\ 0 & 0 \\ 0 & 0 \\ 1 & 0 \\ 0 & 0 \\ 0 & 0 \\ 0 & 0 \\ 394 & 286 \\ 166 & 220 \\ 0 & 0 \\ 0 & 1 \\ 5 & 1 \\ 3 & 1 \\ 2 & 2 \\ 4 & 0 \\ 2 & 1 \\ 0 & 0 \\ 1 & 0\end{array}$

1
1,297
1
0
2
18
1
0
1
0
0
0
1
22
1
1
1
0
1
1
0
450
200
0
0
0
5
0
2
2
0
0

$\begin{array}{rr}3 & 0 \\ 1,486 & 1,279 \\ 0 & 0 \\ 0 & 0 \\ 1 & 1 \\ 42 & 25 \\ 0 & 1 \\ 0 & 0 \\ 1 & 1 \\ 11 & 3 \\ 1 & 0 \\ 3 & 2 \\ 0 & 0 \\ 86 & 35 \\ 16 & 3 \\ 0 & 0 \\ 0 & 1 \\ 0 & 0 \\ 0 & 0 \\ 0 & 0 \\ 0 & 1 \\ 576 & 411 \\ 318 & 258 \\ 1 & 0 \\ 0 & 0 \\ 2 & 4 \\ 3 & 9 \\ 1 & 2 \\ 0 & 1 \\ 5 & 2 \\ 1 & 0 \\ 0 & 0\end{array}$


Table 3.-Benthic macrofauna data-Continued

\begin{tabular}{lccccc}
\hline \multirow{2}{*}{ Taxonomic entry } & Kinnetic & \multicolumn{5}{c}{ Individuals } \\
\cline { 3 - 6 } & Laboratory & \multicolumn{4}{c}{ Replicate number } \\
\cline { 3 - 6 } & code & 1 & 2 & 3 & 4 \\
\hline
\end{tabular}

Coyote Point

Sampling date: November 2, 1988-Continued

\begin{tabular}{|c|c|c|c|c|c|}
\hline Molgula manhattensis ....... & 0 & 8 & 0 & 0 & 0 \\
\hline Musculista senhousia & 5 & 10 & 11 & 22 & 6 \\
\hline Mysella sp. (A) (SCAMIT) . . . . . . . . . .5540212 & 0 & 0 & 0 & 1 & 1 \\
\hline Mytilus edulis ................5540024 & 0 & 0 & 0 & 1 & 0 \\
\hline Natantia, unident. . . . . . . . . . . . 55286111 & 1 & 0 & 0 & 0 & 0 \\
\hline Neanthes succinea ...............4810562 & 2 & 1 & 0 & 4 & 0 \\
\hline Nematodes, unident. . .............4500001 & 50 & 62 & 63 & 132 & 61 \\
\hline Nephtys caecoides ...............4810114 & 0 & 1 & 1 & 1 & 0 \\
\hline Nephtys cornuta franciscana . .........4810116 & 1 & 2 & 4 & 2 & 8 \\
\hline Nephtys $\mathrm{sp}(\mathrm{p}) . \ldots \ldots \ldots \ldots \ldots \ldots . \ldots 4810579$ & 3 & 0 & 0 & 0 & 0 \\
\hline Odostomia (Evalea) sp. H (Shrake) _....5570314 & 4 & 2 & 5 & 6 & 6 \\
\hline Odostomia (Evalea) sp. J (Shrake) ......5570305 & 1 & 0 & 0 & 0 & 0 \\
\hline Odostomia $\mathrm{sp}(\mathrm{p}) . \ldots \ldots \ldots \ldots \ldots \ldots 5570075$ & 1 & 0 & 0 & 0 & 0 \\
\hline Oligochaete, unident. . ............4880001 & 24 & 38 & 47 & 101 & 34 \\
\hline Philine sp. (A) (SCAMIT) ...........5570240 & 2 & 0 & 2 & 1 & 1 \\
\hline Phoronis sp(p). ...............5700002 & 1 & 1 & 0 & 0 & 0 \\
\hline Polydora ligni .................4810168 & 130 & 69 & 117 & 168 & 110 \\
\hline Potamocorbula amurensis ..........5540214 & 38 & 31 & 49 & 41 & 40 \\
\hline Pseudopolydora kempi ............4810640 & 6 & 3 & 3 & 12 & 3 \\
\hline Pseudopolydora paucibranchiata . .....4810347 & 23 & 5 & 36 & 60 & 32 \\
\hline Pyromaia tuberculata .............5286094 & 0 & 0 & 0 & 3 & 2 \\
\hline Sarsiella zostericola .............5220091 & 13 & 4 & 6 & 10 & 3 \\
\hline Schistomeringos rudolphi ..........4810354 & 1 & 3 & 0 & 8 & 3 \\
\hline Sphaerosyllis californiensis ........4810272 & 3 & 2 & 4 & 17 & 5 \\
\hline Spiophanes bombyx ..............4810203 & 0 & 0 & 0 & 2 & 0 \\
\hline Streblospio benedicti .............4810257 & 0 & 2 & 0 & 0 & 0 \\
\hline Synidotea laticauda ..............55265110 & 0 & 0 & 0 & 1 & 0 \\
\hline Tapes japonica ................5540158 & 23 & 14 & 40 & 33 & 33 \\
\hline Theora lubrica ..................5540114 & 42 & 48 & 28 & 43 & 26 \\
\hline$\ldots \ldots \ldots \ldots \ldots 4000013$ & 0 & 0 & 1 & 0 & 0 \\
\hline
\end{tabular}

Grizzly Bay

Sampling date: January 11,1988

Ampelisca abdita ................. 5275504

Grandidierella japonica ...........5275503

Leucon subnasica ...............55263012

Macoma balthica ................5540147

Neanthes succinea .................4810562

Oligochaete, unident. ............44880001

Potamocorbula amurensis ..........5540214

$\begin{array}{rr}0 & 1 \\ 0 & 0 \\ 8 & 17 \\ 2 & 0 \\ 0 & 1 \\ 16 & 19 \\ 176 & 158\end{array}$

$\begin{array}{rrr}0 & 1 & 0 \\ 0 & 1 & 0 \\ 8 & 57 & 32 \\ 0 & 0 & 0 \\ 1 & 1 & 0 \\ 20 & 24 & 22 \\ 187 & 192 & 141\end{array}$


Table 3.-Benthic macrofauna data-Continued

\begin{tabular}{|c|c|c|c|c|c|c|}
\hline \multirow{3}{*}{ Taxonomic entry } & \multirow{3}{*}{$\begin{array}{c}\text { Kinnetic } \\
\text { Laboratory } \\
\text { code }\end{array}$} & \multicolumn{5}{|c|}{ Individuals } \\
\hline & & \multicolumn{5}{|c|}{ Replicate number } \\
\hline & & 1 & 2 & 3 & 4 & $\overline{5}$ \\
\hline
\end{tabular}

Grizzly Bay

Sampling date: January 11,1988 -Continued

Pseudopolydora kempi ............4810640

Streblospio benedicti

4810257

\section{0}

0

Sampling date: March 14, 1988
Ampelisca abdita .................55275504

Exogone lourei ................44810066

Leucon subnasica .................55263012

Macoma balthica .................55540147

Neanthes succinea ................4810562

Oligochaete, unident. .............4880001

Potamocorbula amurensis ..........5540214

Pseudopolydora kempi .............4810640

Sarsiella zostericola ...............5220091

Streblospio benedicti ..............44810257

$\begin{array}{rr}2 & 0 \\ 1 & 0 \\ 9 & 29 \\ 0 & 2 \\ 1 & 0 \\ 12 & 37 \\ 151 & 276 \\ 0 & 1 \\ 1 & 0 \\ 0 & 2\end{array}$

$\begin{array}{rrr}0 & 0 & 1 \\ 0 & 0 & 0 \\ 16 & 16 & 34 \\ 0 & 2 & 0 \\ 1 & 0 & 1 \\ 25 & 30 & 25 \\ 131 & 201 & 248 \\ 0 & 1 & 0 \\ 0 & 0 & 0 \\ 3 & 3 & 0\end{array}$

Sampling date: May 23, 1988

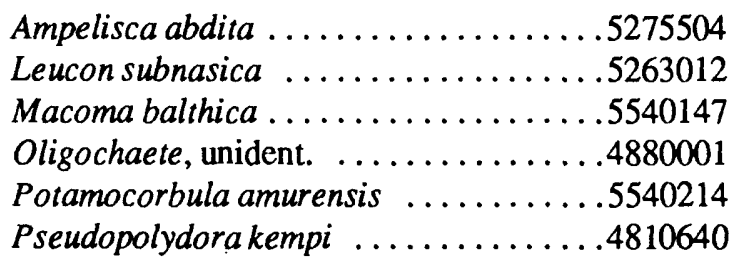

Ampelisca abdita .................5275504

Leucon subnasica .................55263012

Oligochaete, unident. ..................5540147

Potamocorbula amurensis ..........5540214

Pseudopolydora kempi ............4810640

$\begin{array}{rr}0 & \\ 6 & 17 \\ 1 & \\ 2 & \\ 153 & 135 \\ 0 & \end{array}$

1

0

Sampling date: July 21, 1988

Balanus improvisus . . . . . . . . . . . . . 5250020

Brachyuran zoea .................55286063

Corophium sp. a ................5275510

Leucon subnasica ................5263012

Neanthes succinea .................44810562

Oligochaete, unident. ............4880001

Potamocorbula amurensis ..........5540214

$\begin{array}{rr}2 & \\ 0 & \\ 1 & \\ 0 & \\ 0 & \\ 0 & \\ 76 & 47\end{array}$
1
0
0
1
0

2

47

$\begin{array}{rr}0 & 0 \\ 5 & 28 \\ 1 & 1 \\ 3 & 8 \\ 141 & 188 \\ 1 & 0\end{array}$

Sampling date: September 12, 1988

Ampelisca abdita ..................5275504

Balanus improvisus ..............5250020

Corophium sp(p)................. 5275098

Heteromastus filiformis . . . . . . . . . . .4810438

Hydrozoa, unident. .................3710052

Leucon subnasica ...............5263012
0

2

0

1

0

0 
Table 3.-Benthic macrofauna data-Continued

\begin{tabular}{lccccc}
\hline \multirow{2}{*}{ Taxonomic entry } & Kinnetic & \multicolumn{5}{c}{ Individuals } \\
\cline { 2 - 6 } & Laboratory & \multicolumn{4}{c}{ Replicate number } \\
\cline { 2 - 6 }
\end{tabular}

\section{Grizzly Bay}

Sampling date: September 12, 1988-Continued

Neanthes succinea . . . . . . . . . . . 4810562

Nematodes, unident. ............4500001

Oligochaete, unident. ............44880001

Potamocorbula amurensis .........5540214

Pseudopolydora kempi ... . . . . . . . .4810640

Streblospio benedicti .............4810257

Volvulella panamica ............ 5570236

$\begin{array}{rr}0 & 0 \\ 0 & 0 \\ 0 & 0 \\ 182 & 141 \\ 0 & 1 \\ 0 & 0 \\ 1 & 0\end{array}$

Sampling date: October 31, 1988

Ampelisca abdita ... . . . . . . . . . .55275504

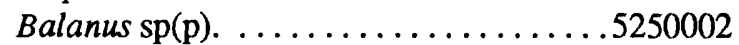

Diopatra $\mathrm{sp}(\mathrm{p}) . \ldots . . \ldots . . . . . . .44810999$

Exogone lourei . . . . . . . . . . . . 4810066
Grandidierella japonica . . . . . . . . 5275503

Heteromastus filiformis . . . . . . . . . . . .4810438

Leucon subnasica . . . . . . . . . . . . .5263012

Neanthes succinea . . . . . . . . . . . . 4810562

Nematodes, unident. . . . . . . . . . . 4500001

Oligochaete, unident. . . . . . . . . . . 4880001

Potamocorbula amurensis . . . . . . . 5540214

Pseudopolydora kempi .............4810640

$\begin{array}{rr}1 & 1 \\ 10 & 2 \\ 0 & 0 \\ 0 & 2 \\ 0 & 1 \\ 0 & 0 \\ 6 & 9 \\ 0 & 0 \\ 0 & 0 \\ 7 & 0 \\ 236 & 197 \\ 0 & 0\end{array}$

$\begin{array}{rrr}0 & 1 & 0 \\ 0 & 0 & 1 \\ 0 & 3 & 2 \\ 161 & 212 & 251 \\ 1 & 2 & 1 \\ 0 & 1 & 2 \\ 0 & 0 & 0\end{array}$

\section{$\underline{\text { Palo Alto }}$}

Sampling date: January 14, 1988

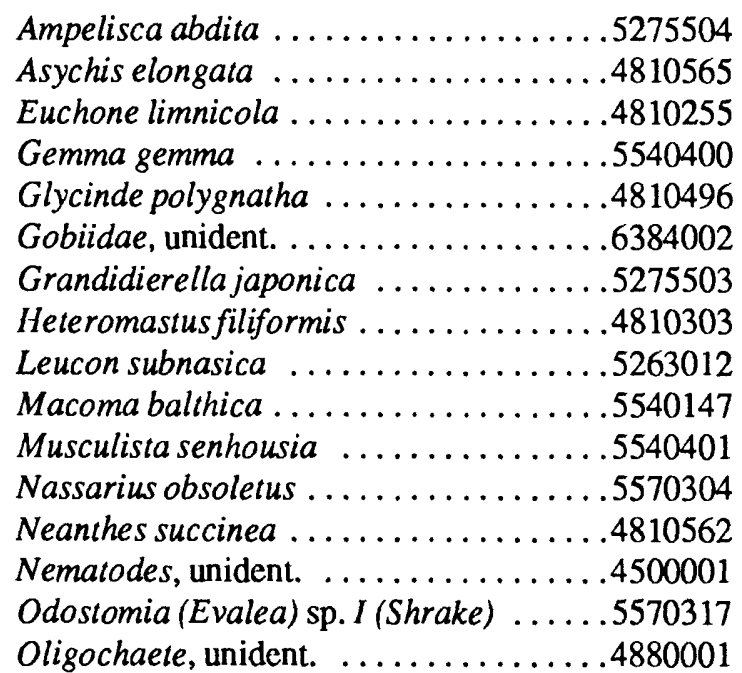

$\begin{array}{rr}107 & 439 \\ 3 & 5 \\ 1 & 2 \\ 1,042 & 1,459 \\ 6 & 9 \\ 1 & 0 \\ 1 & 1 \\ 26 & 93 \\ 59 & 65 \\ 2 & 1 \\ 5 & 7 \\ 0 & 1 \\ 4 & 3 \\ 3 & 15 \\ 14 & 23 \\ 35 & 107\end{array}$

956
2
2
1,530
12
0
3
52
64
1
9
0
1
42
34
93

556

3

1

1,862

10

0

2

81

76

3

11

0

2

7

18

61 
Table 3.-Benthic macrofauna data-Continued

\begin{tabular}{|c|c|c|c|c|c|}
\hline \multirow{3}{*}{ Taxonomic entry } & \multirow{3}{*}{$\begin{array}{c}\text { Kinnetic } \\
\text { Laboratory } \\
\text { code }\end{array}$} & \multicolumn{4}{|c|}{ Individuals } \\
\hline & & \multicolumn{4}{|c|}{ Replicate number } \\
\hline & & 1 & 3 & 4 & 5 \\
\hline
\end{tabular}

$\underline{\text { Palo Alto }}$

Sampling date: January 14, 1988 - Continued

Pseudopolydora kempi ............4810640

Sarsiella zostericola .............55220091

Streblospio benedicti ..............4810257

Synidotea laticauda ...............5265110

Tharyx sp.

$\begin{array}{rr}2 & \\ 127 & 20 \\ 65 & 98 \\ 0 & \\ 2 & \end{array}$

0
205
98
1
5

0
295
122
0
1

0
269
102
0
2

0

224

105

0

Sampling date: March 7, 1988

Ampelisca abdita ................5275504

Asychis elongata ...............4810565

Campylaspis sp(p). .............5263001

Cumella vulgaris ................55263098

Euchone limnicola ................4810255

Gemma gemma ..................5540400

Glycinde polygnatha . . ............4810496

Harmothoe $\mathrm{sp(p).................4810856}$

Heteromastus filiformis . . . . . . . . . . .4810438

Leucon subnasica ...............55263012

Macoma balthica ................... 5540147

Musculista senhousia .............5540401

Neanthes succinea . . . . . . . . . . . . . 4810562

Nematodes, unident. .............4500001

Nephtys caecoides ...............4810114

Odostomia (Evalea) sp. H (Shrake) ....5570314

Odostomia (Evalea) sp. J (Shrake) . . . . . 5570305

Oligochaete, unident. ..............4880001

Polydora ligni ....................44810168

Potamocorbula amurensis . . . . . . . . . 5540214

Pseudopolydora kempi ............4810640

Sarsiella zostericola .............5220091

Sphaerosyllis bilobata ............44810833

Streblospio benedicti ..............4810257

Tharyx sp(p). ...................4810319

Upogebia pugettensis ...........55286103

$\begin{array}{rr}147 & 150 \\ 3 & 1 \\ 0 & 1 \\ 28 & 10 \\ 2 & 5 \\ 546 & 985 \\ 4 & 2 \\ 4 & 0 \\ 62 & 42 \\ 157 & 56 \\ 0 & 0 \\ 7 & 9 \\ 3 & 5 \\ 9 & 2 \\ 0 & 1 \\ 4 & 6 \\ 1 & 0 \\ 70 & 29 \\ 7 & 4 \\ 0 & 1 \\ 2 & 0 \\ 138 & 74 \\ 3 & 0 \\ 209 & 54 \\ 0 & 0 \\ 2 & 0\end{array}$

228
2
2
37
2
513
4
3
45
218
0
1
1
2
0
1
0
40
7
0
1
94
2
171
5
1

176

0

7

719

1

2

93

194

3

6

2

3

0

2

0

100

14

0

0
144

2

277

4

0

3,190

2,735

Ampelisca abdita ...............5275504

Amphiurid, unident. ............... 5930014

Anthozoan, unident. . . . . . . . . . . . 3730010

Asychis elongata ............... 4810565

Cirratulidae, unident. . . . . . . . . . . 4810990

Corophium sp(p). . . . . . . . . . . . .5275098
1,324

0

1

0

0

141
2,910

0

0

1

1
196

196
207

2

0

21

2

783

4

3

96

145

0

7

4

2

0

3

0

91

0

0

2

113

1

212

2

1 
Table 3.-Benthic macrofauna data-Continued

\begin{tabular}{lcccccr}
\hline \multirow{2}{*}{ Taxonomic entry } & Kinnetic & \multicolumn{5}{c}{ Individuals } \\
\cline { 2 - 6 } & Laboratory & \multicolumn{4}{c}{ Replicate number } \\
\cline { 3 - 6 } & code & 1 & 2 & 3 & 4 & 5 \\
\hline
\end{tabular}

$\underline{\text { Palo Alto }}$

Sampling date: May 26, 1988 - Continued

Corophium sp. a ...............5275510

Cumella vulgaris .................55263098

Gemma gemma ..................5540400

Glycinde polygnatha ..............4810496

Grandidierella japonica ...........5275503

Harmothoe imbricata . . . . . . . . . . . . 4810343

Heteromastus filiformis . . . . . . . . . . . 4810438

Leucon subnasica . . . . . . . . . . . . . 5263012

Macoma balthica .................5540147

Macoma sp(p)..................5540105

Musculista senhousia .............5540401

Mya arenaria ...................55540402

Neanthes succinea . . . . . . . . . . . . . . 4810562

Nematodes, unident. . . . . . . . . . . . 44500001

Nemertea, unident. . . . . . . . . . . . . 4000002

Odostomia (Evalea) sp. H (Shrake) ....5570314

Odostomia (Evalea) sp. I (Shrake) . ....5570317

Odostomia (Evalea) sp. J (Shrake) ......5570305

Oligochaete, unident. .............4880001

Philine sp. (A) (SCAMIT) . . . . . . . . . 5570240

Polydora ligni ...................4810168

Potamocorbula amurensis . .........5540214

Pseudopolydora kempi ............4810640

Pseudopolydora paucibranchiata . . . . . .4810347

Sarsiella zostericola .............5220091

Streblospio benedicti ..............4810257

Tapes japonica ..................5540158

Theora lubrica ....................55540114

Upogebia pugettensis ............5286103

$\begin{array}{rr}1 & 1 \\ 20 & 8 \\ 1,368 & 1,699 \\ 46 & 74 \\ 34 & 15 \\ 4 & 3 \\ 6 & 3 \\ 142 & 137 \\ 4 & 3 \\ 0 & 0 \\ 8 & 12 \\ 3 & 7 \\ 1 & 2 \\ 24 & 0 \\ 0 & 0 \\ 0 & 1 \\ 6 & 4 \\ 5 & 11 \\ 14 & 7 \\ 5 & 10 \\ 1 & 0 \\ 14 & 10 \\ 1 & 1 \\ 26 & 9 \\ 197 & 118 \\ 24 & 22 \\ 0 & 0 \\ 11 & 12 \\ 0 & 0\end{array}$

$\begin{array}{rrr}0 & 1 & 0 \\ 39 & 27 & 37 \\ 964 & 1,128 & 974 \\ 87 & 72 & 80 \\ 37 & 67 & 33 \\ 14 & 5 & 10 \\ 13 & 3 & 8 \\ 167 & 143 & 136 \\ 5 & 3 & 0 \\ 0 & 0 & 1 \\ 8 & 8 & 17 \\ 2 & 4 & 1 \\ 1 & 0 & 1 \\ 15 & 4 & 4 \\ 4 & 0 & 0 \\ 7 & 0 & 6 \\ 0 & 3 & 0 \\ 4 & 4 & 0 \\ 20 & 8 & 10 \\ 1 & 15 & 4 \\ 1 & 0 & 0 \\ 12 & 7 & 7 \\ 0 & 0 & 0 \\ 14 & 8 & 1 \\ 186 & 237 & 196 \\ 23 & 65 & 18 \\ 0 & 1 & 0 \\ 9 & 9 & 4 \\ 1 & 0 & 0\end{array}$

Sampling date: July 26,1988

Ampelisca abdita ...............5275504

Asychis elongata ................4810565

Cirratulidae, unident. .............4810990

Corophium $\mathrm{sp}(\mathrm{p})$. . . . . . . . . . . . . . . 5275098

Euchone limnicola ...............44810255

Exogone lourei ................4810066

Gemma gemma ....................5540400

Glycinde polygnatha ................4810496

Grandidierella japonica ............5275503

Harmothoe imbricata . . . . . . . . . . . 4810343

Heteromastus filiformis . . . . . . . . . .4810438

452
2
0
148
0
1
448
16
202
4
13

589

0

1

96

0

0

559

15

173

5

33
537 
Table 3.-Benthic macrofauna data-Continued

\begin{tabular}{|c|c|c|c|c|c|}
\hline \multirow{3}{*}{ Taxonomic entry } & \multirow{3}{*}{$\begin{array}{l}\text { Kinnetic } \\
\text { Laboratory } \\
\text { code }\end{array}$} & \multicolumn{4}{|c|}{ Individuals } \\
\hline & & \multicolumn{4}{|c|}{ Replicate number } \\
\hline & & 1 & 3 & 4 & 5 \\
\hline
\end{tabular}

$\underline{\text { Palo Alto }}$

Sampling date: July 26, 1988 - Continued

Leucon subnasica . . . . . . . . . . . .5263012

Lumbrineris $\mathrm{sp}(\mathrm{p}) \ldots \ldots \ldots \ldots \ldots \ldots . \ldots 4810584$

Macoma sp(p)...................5540105

Melanochlamys diomedea . . . . . . . . . 5570248

Musculista senhousia .............5550401

Mya arenaria ..................55540402

Neanthes succinea ...............44810562

Nematodes, unident. .............4500001

Odostomia (Evalea) sp. I (Shrake) . . . ...5570317

Odostomia (Evalea) sp. J (Shrake) . . . . . 5570305

Philine sp. (A) (SCAMIT) . . . . . . . . 5570240

Potamocorbula amurensis . . . . . . . . 5540214

Pseudopolydora kempi ............. 4810640

Pseudopolydora paucibranchiata ..... . 4810347

Sarsiella zostericola ..............5220091

Streblospio benedicti .............. 4810257

Synidotea laticauda ...............55265110

Tapes japonica .................5540158

Theora lubrica . . . . . . . . . . . . . . . . . .5540114

$\begin{array}{rrrrr}26 & 11 & 39 & 47 & 30 \\ 1 & 0 & 0 & 0 & 0 \\ 0 & 2 & 0 & 6 & 0 \\ 0 & 0 & 0 & 0 & 1 \\ 11 & 9 & 8 & 8 & 5 \\ 4 & 1 & 2 & 2 & 1 \\ 0 & 2 & 0 & 0 & 1 \\ 0 & 0 & 3 & 2 & 0 \\ 2 & 0 & 0 & 0 & 1 \\ 1 & 1 & 0 & 0 & 1 \\ 4 & 3 & 2 & 8 & 3 \\ 12 & 11 & 14 & 11 & 7 \\ 1 & 3 & 3 & 1 & 2 \\ 0 & 0 & 8 & 0 & 0 \\ 289 & 268 & 318 & 362 & 373 \\ 13 & 7 & 33 & 34 & 39 \\ 0 & 0 & 2 & 0 & 0 \\ 0 & 4 & 0 & 5 & 1 \\ 22 & 12 & 17 & 21 & 5\end{array}$

Sampling date: September 14, 1988

Ampelisca abdita ................. 5275504

Asychis elongata ...............4810565

Caprella sp(p)..................5275117

Cirratulidae, unident. . . . . . . . . . . . 4810990

Corophium sp(p)...................55275098

Deutella californica ..............5275261

Exogone lourei ................4810066

Gemma gemma .................5540400

Glycinde polygnatha .............4810496

Grandidierella japonica ...........5275503

Harmothoe imbricata .............4810343

Harmothoe lunulata .............44810689

Heteromastus filiformis . . . . . . . . . . .4810438

Leucon subnasica ...............55263012

Macoma sp(p)..................5540105

Musculista senhousia .............5540401

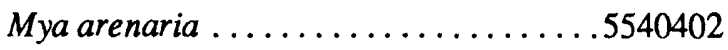

Nassarius obsoletus . . . . . . . . . . . .5570304

Neanthes succinea . . . . . . . . . . . . . 4810562

Nematodes, unident. ...............4500001

Nephtys cornuta franciscana ..........4810116

$\begin{array}{rr}1,963 & 3,316 \\ 0 & 1 \\ 0 & 1 \\ 0 & 2 \\ 1 & 0 \\ 0 & 1 \\ 0 & 0 \\ 914 & 528 \\ 6 & 4 \\ 62 & 52 \\ 0 & 2 \\ 0 & 1 \\ 22 & 30 \\ 213 & 237 \\ 0 & 1 \\ 11 & 17 \\ 4 & 1 \\ 0 & 1 \\ 2 & 6 \\ 4 & 9 \\ 0 & 0\end{array}$

$\begin{array}{rr}4,127 & 2,686 \\ 3 & 1 \\ 0 & 0 \\ 0 & 3 \\ 10 & 0 \\ 2 & 0 \\ 3 & 0 \\ 392 & 236 \\ 5 & 8 \\ 71 & 43 \\ 1 & 1 \\ 0 & 0 \\ 15 & 26 \\ 234 & 136 \\ 2 & 2 \\ 11 & 4 \\ 1 & 2 \\ 0 & 0 \\ 9 & 2 \\ 52 & 133 \\ 0 & 2\end{array}$


Table 3.-Benthic macrofauna data-Continued

\begin{tabular}{lcccccc}
\hline \multirow{2}{*}{ Taxonomic entry } & Kinnetic & \multicolumn{5}{c}{ Individuals } \\
\cline { 2 - 6 } & Laboratory & \multicolumn{4}{c}{ Replicate number } \\
\cline { 2 - 6 } & code & 1 & 2 & 3 & 4 & 5 \\
\hline
\end{tabular}

Palo Alto

Sampling date: September 14, 1988-Continued

\begin{abstract}
Nudibranchia, unident. . . . . . . . . . . 5570976
Odostomia (Evalea) sp. I (Shrake) ..... 5570317

Odostomia (Evalea) sp. J (Shrake) ......5570305

Oligochaete, unident. ..............4880001

Philine sp. (A) (SCAMIT) ... . . . . . . 5570240

Podocopid ostracod ................. 5220092

Polydora ligni ..................4810168

Polydora socialis . .................4810940

Potamocorbula amurensis ..........5540214

Pseudopolydora kempi ..............4810640

Pseudopolydora paucibranchiata . . . . . . 4810347

Sarsiella zostericola ..............5220091

Silophasma geminata .............5265020

Streblospio benedicti ...............4810257

Synidotea laticauda ................ 5265110

Tapes japonica .................. 5540158

Theora lubrica ................... 5540114
\end{abstract}

$\begin{array}{rr}0 & 7 \\ 6 & 7 \\ 0 & 2 \\ 87 & 143 \\ 11 & 6 \\ 0 & 1 \\ 29 & 31 \\ 0 & 0 \\ 81 & 71 \\ 8 & 5 \\ 20 & 13 \\ 284 & 282 \\ 0 & 1 \\ 59 & 108 \\ 0 & 2 \\ 7 & 7 \\ 14 & 11\end{array}$

0
0
1
187
7
0
42
0
38
9
18
241
0
158
0
6
12

$\begin{array}{rr}0 & 0 \\ 3 & 1 \\ 0 & 0 \\ 126 & 207 \\ 6 & 6 \\ 0 & 1 \\ 41 & 13 \\ 3 & 1 \\ 63 & 26 \\ 3 & 11 \\ 23 & 16 \\ 319 & 184 \\ 0 & 0 \\ 91 & 43 \\ 0 & 0 \\ 4 & 2 \\ 10 & 8\end{array}$

Sampling date: November 2, 1988

Ampelisca abdita ................. 5275504

Asychis elongata .................4810565

Cirratulidae, unident. . . . . . . . . . . . .4810990

Euchone limnicola .................4810255

Eudorella pacifica ................5263112

Gemma gemma ..................5540400

Glycinde polygnatha ..............4810496

Grandidierella japonica ............5275503

Harmothoe imbricata ..............4810343

Heteromastus filiformis . . . . . . . . . . .4810438

Leucon subnasica ................55263012

Macoma $\mathrm{sp}(\mathrm{p}) . \ldots \ldots \ldots \ldots \ldots . . . . . .5540105$

Melanochlamys diomedea . . . . . . . . . . .5570248

Musculista senhousia ..............5540401

Mya arenaria ...................5540402

Nassarius obsoletus . . . . . . . . . . . . . 5570304

Neanthes succinea ................4810562

Nematodes, unident. .............4500001

Odostomia (Evalea) sp. I (Shrake) ......5570317

Odostomia (Evalea) sp. J (Shrake) ...... 5570305

Odostomia (Evalea) sp. $N$ (Shrake) .....5570321

Oligochaete, unident. .............4880001

Philine sp. (A) (SCAMIT) ...........55570240

$\begin{array}{rr}1,783 & 2,297 \\ 1 & 0 \\ 0 & 0 \\ 3 & 2 \\ 0 & 1 \\ 237 & 390 \\ 7 & 5 \\ 30 & 43 \\ 1 & 4 \\ 20 & 17 \\ 103 & 121 \\ 0 & 0 \\ 0 & 0 \\ 33 & 45 \\ 0 & 1 \\ 0 & 0 \\ 3 & 3 \\ 22 & 21 \\ 0 & 0 \\ 1 & 7 \\ 0 & 1 \\ 110 & 48 \\ 0 & 3\end{array}$

$\begin{array}{rr}2,253 & 1,271 \\ 0 & 0 \\ 0 & 5 \\ 0 & 2 \\ 5 & 0 \\ 310 & 361 \\ 3 & 2 \\ 29 & 30 \\ 0 & 1 \\ 4 & 32 \\ 71 & 82 \\ 0 & 1 \\ 0 & 1 \\ 28 & 40 \\ 0 & 0 \\ 0 & 0 \\ 4 & 4 \\ 87 & 7 \\ 6 & 4 \\ 10 & 4 \\ 0 & 0 \\ 35 & 71 \\ 2 & 2\end{array}$


Table 3.-Benthic macrofauna data-Continued

\begin{tabular}{lccccc}
\hline \multirow{2}{*}{ Taxonomic entry } & Kinnetic & \multicolumn{5}{c}{ Individuals } \\
\cline { 2 - 6 } & Laboratory & \multicolumn{4}{c}{ Replicate number } \\
\cline { 2 - 6 } & code & 1 & 2 & 3 & 4 \\
\hline
\end{tabular}

$\underline{\text { Palo Alto }}$

Sampling date: November 2, 1988-Continued

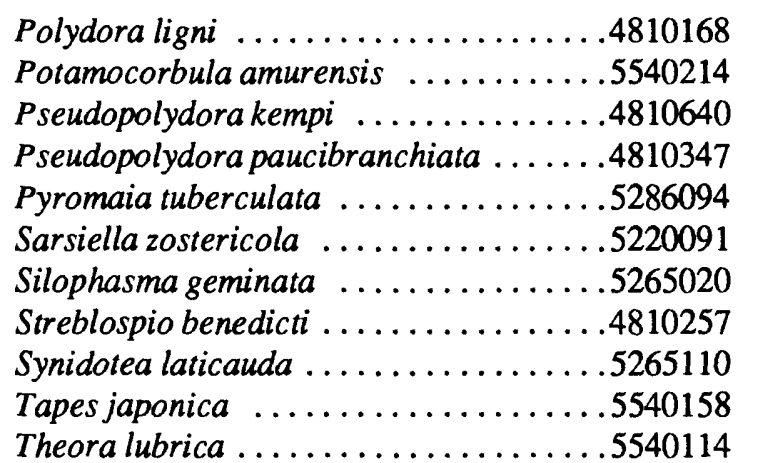

$\underline{\text { San Leandro }}$

Sampling date: January 13, 1988
Ampelisca abdita ................ 5275504

Anthozoan, unident. ............... 3730010

Asychis elongata ................4810565

Cirratulidae, unident. . . . . . . . . . . 4810990

Corophium alienense ..............55275287

Corophium sp(p).................5275098

Cossura pygodactylata .............4810861

Crepidula plana .................5570204

Crepidula sp(p)..................5570203

Crepidula sp. A (Shrake) ............ 5570331

Euchone limnicola ...............4810255

Exogone lourei ...............4810066

Glycinde polygnatha . . . . . . . . . . . 4810496

Grandidierella japonica ...........5275503

Harmothoe imbricata ..............4810343

Heteromastus filiformis . . . . . . . . . . .44810438

Macoma balthica ................ 5540147

Marphysa sanguinea ............. 4810248

Musculista senhousia ..............5540401

Nematodes, unident. ...............4500001

Nephtys cornuta franciscana . . . . . . . . .4810116

Oligochaete, unident. .............44880001

Paranemertes sp(p). ..............4000050

Polydora brachycephala ............ 4810557

Pseudopolydora kempi ............4810640

Pseudopolydora paucibranchiata ...... 4810347

Sarsiella zostericola .............5220091

$\begin{array}{rrrrr}0 & 1 & 0 & 0 & 1 \\ 56 & 83 & 68 & 57 & 61 \\ 0 & 0 & 1 & 0 & 1 \\ 0 & 4 & 1 & 0 & 0 \\ 0 & 0 & 0 & 0 & 1 \\ 336 & 487 & 388 & 246 & 393 \\ 0 & 4 & 1 & 1 & 5 \\ 34 & 5 & 28 & 20 & 11 \\ 0 & 0 & 0 & 0 & 1 \\ 3 & 7 & 2 & 6 & 6 \\ 3 & 9 & 4 & 5 & 3\end{array}$

1 61 1

0 1

3

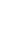


Table 3.-Benthic macrofauna data-Continued

\begin{tabular}{|c|c|c|c|c|c|c|}
\hline \multirow{2}{*}{ Taxonomic entry } & \multirow{3}{*}{$\begin{array}{c}\text { Kinnetic } \\
\text { Laboratory } \\
\text { code }\end{array}$} & \multicolumn{5}{|c|}{ Individuals } \\
\hline & & \multicolumn{5}{|c|}{ Replicate number } \\
\hline & & 1 & 2 & 3 & 4 & 5 \\
\hline
\end{tabular}

$\underline{\text { San Leandro }}$

Sampling date: January 13,1988 - Continued

Schistomeringos rudolphi ..........44810354

Sphaerosyllis bilobata .............44810833

Sphaerosyllis californiensis .........4810272

Tapes japonica .................5540158

Tenonia priops ..................4810727

Theora lubrica ...................55540114

Urosalpinx cinerea ..............5570200
0

0

3

6

0

0

0

$\begin{array}{rrr}0 & 3 & 0 \\ 8 & 2 & 9 \\ 11 & 5 & 9 \\ 8 & 3 & 13 \\ 1 & 1 & 1 \\ 2 & 1 & 1 \\ 1 & 1 & 0\end{array}$

Sampling date: March 8, 1988

\begin{tabular}{|c|c|c|c|c|}
\hline Ampelisca abdita ...............5275504 & 11 & 2 & 2 & 5 \\
\hline Anthozoan, unident. . .............3730010 & 1 & 2 & 0 & 2 \\
\hline Asychis elongata ..............4810565 & 4 & 5 & 3 & 6 \\
\hline Crepidula plana ..................5570204 & 0 & 3 & 0 & 3 \\
\hline Crepidula sp. A (Shrake) . . . . . . . . . . .5570331 & 5 & 0 & 1 & 3 \\
\hline ..............4810066 & 2 & 15 & 9 & 27 \\
\hline Gemma gemma ...............55540400 & 1 & 4 & 1 & 0 \\
\hline Glycinde polygnatha ..............4810496 & 5 & 8 & 5 & 8 \\
\hline Harmothoe imbricata ............ 4810343 & 0 & 0 & 1 & 0 \\
\hline Hemigrapsus oregonensis . . . . . . . . . 5286092 & 0 & 0 & 0 & 0 \\
\hline Heteromastus filiformis . . . . . . . . . . . 4810438 & 0 & 0 & 1 & 1 \\
\hline Lightiella serendipita ............5204001 & 0 & 0 & 0 & 1 \\
\hline Macoma $\mathrm{sp}(\mathrm{p}) \ldots \ldots \ldots \ldots \ldots \ldots \ldots 5540105$ & 1 & 0 & 0 & 0 \\
\hline Marphysa sanguinea ............4810248 & 5 & 2 & 2 & 4 \\
\hline Musculista senhousia ............5540401 & 25 & 67 & 23 & 45 \\
\hline Nematodes, unident. ............4500001 & 45 & 33 & 7 & 110 \\
\hline Oligochaete, unident. ............4880001 & 11 & 37 & 26 & 40 \\
\hline Ostrea edulis .................5540215 & 0 & 0 & 1 & 0 \\
\hline Pettiboneia sanmatiensis . . . . . . . . . 4810552 & 0 & 0 & 0 & 0 \\
\hline Polydora brachycephala ............4810557 & 0 & 0 & 0 & 0 \\
\hline Pseudopolydora paucibranchiata . ..... 4810347 & 1 & 0 & 0 & 0 \\
\hline Sarsiella zostericola .............5220091 & 18 & 21 & 6 & 23 \\
\hline Schistomeringos rudolphi ..........4810354 & 0 & 0 & 0 & 0 \\
\hline Sphaerosyllis bilobala ............4810833 & 0 & 9 & 0 & 4 \\
\hline Sphaerosyllis californiensis ........4810272 & 1 & 4 & 3 & 4 \\
\hline Tapes japonica ...............5540158 & 6 & 19 & 12 & 22 \\
\hline Theora lubrica ...................5540114 & 0 & 2 & 0 & 0 \\
\hline Tubulanus $\mathrm{sp}(\mathrm{p}) . \ldots \ldots \ldots \ldots \ldots . . . .4000013$ & 0 & 0 & 1 & 0 \\
\hline
\end{tabular}

Sampling date: May 25, 1988

Amaeana occidentalis .............44810001

Ampelisca abdita

\section{0}

5

\section{0}

26

\section{0}

11
0
9
9
3
1
1
0 
Table 3. Benthic macrofauna data-Continued

\begin{tabular}{|c|c|c|c|c|c|c|}
\hline \multirow{2}{*}{ Taxonomic entry } & \multirow{3}{*}{$\begin{array}{c}\text { Kinnetic } \\
\text { Laboratory } \\
\text { code }\end{array}$} & \multicolumn{5}{|c|}{ Individuals } \\
\hline & & \multicolumn{5}{|c|}{ Replicate number } \\
\hline & & 1 & 2 & 3 & 4 & 5 \\
\hline
\end{tabular}

$\underline{\text { San Leandro }}$

Sampling date: May 25, 1988 — Continued

Anthozoan, unident. . . . . . . . . . . . 3730010

Asychis elongata ................4810565

Balanus sp(p). ................5250002

Brachyuran megalops ............. 5286064

Cirratulidae, unident. . . . . . . . . . . . 4810990

Corophium sp(p)..................5275098

Corophium sp. a ...............55275510

Cossura pygodactylata .............4810861

Crepidula sp(p)...................5570203

Crepidula sp. A (Shrake) ............ 5570331

Exogone lourei .................4810066

Glycinde polygnatha .............4810496

Harmothoe imbricata . . . . . . . . . . . . 4810343

Heptacarpus stimpsoni . ...........5286106

Leucon subnasica ................55263012

Marphysa sanguinea .............4810248

Musculista senhousia ............... 5540401

Mysella sp. (A) (SCAMIT) . . . . . . . . . . 5540212

Mysella tumida .................... 5540145

Mytilidae, unident. . . . . . . . . . . . . . . . 5540403

Nematodes, unident. ..............4500001

Nephtys cornuta franciscana . . . . . . . . . 4810116

Oligochaete, unident. ............4880001

Philine sp. (A) (SCAMIT) . . . . . . . . . 5570240

Porifera ...................... 3600000

Pyromaia tuberculata .............. 5286094

Sarsiella zostericola ...............5220091

Schistomeringos rudolphi ...........4810354

Sphaerosyllis bilobata .............4810833

Sphaerosyllis californiensis ........4810272

Tapes japonica .................55540158

Theora lubrica ................... 5540114

$\begin{array}{rrrrr}0 & 0 & 0 & 0 & 10 \\ 1 & 9 & 2 & 0 & 4 \\ 0 & 0 & 0 & 0 & 1 \\ 0 & 1 & 0 & 0 & 0 \\ 1 & 0 & 1 & 0 & 0 \\ 1 & 2 & 0 & 1 & 4 \\ 32 & 102 & 0 & 41 & 5 \\ 0 & 0 & 0 & 0 & 1 \\ 1 & 2 & 0 & 2 & 0 \\ 0 & 0 & 0 & 0 & 8 \\ 12 & 10 & 2 & 8 & 27 \\ 10 & 19 & 5 & 21 & 12 \\ 1 & 0 & 0 & 0 & 6 \\ 0 & 0 & 0 & 0 & 1 \\ 0 & 0 & 0 & 2 & 0 \\ 6 & 3 & 1 & 0 & 1 \\ 18 & 7 & 0 & 1 & 185 \\ 0 & 0 & 0 & 0 & 3 \\ 0 & 0 & 0 & 1 & 0 \\ 0 & 0 & 1 & 0 & 0 \\ 0 & 1 & 0 & 0 & 10 \\ 2 & 6 & 8 & 9 & 0 \\ 13 & 35 & 10 & 28 & 17 \\ 1 & 0 & 9 & 11 & 0 \\ 1 & 0 & 0 & 0 & 1 \\ 1 & 0 & 0 & 0 & 0 \\ 13 & 19 & 11 & 8 & 9 \\ 0 & 2 & 0 & 0 & 0 \\ 1 & 0 & 0 & 0 & 1 \\ 2 & 1 & 2 & 5 & 3 \\ 0 & 0 & 0 & 0 & 8 \\ 1 & 1 & 0 & 2 & 0\end{array}$

Sampling date: July 27, 1988

Ampelisca abdita .................5275504

Anthozoan, unident. . . . . . . . . . . . . 3730010

Asychis elongata ................4810565

Capitella capitata ...............4810241

Corophium sp(p). . . . . . . . . . . . . . 5275098

Corophium sp. a ...............5275510

Cossura pygodactylata ...........4810861

Crepidula $\mathrm{sp}(\mathrm{p}) . \ldots \ldots \ldots \ldots \ldots . \ldots . . .5570203$

$\begin{array}{rr}5 & \\ 0 & 2 \\ 1 & \\ 0 & \\ 0 & \\ 20 & \\ 0 & \\ 3 & 12\end{array}$

10

4

0

4

5

0

27

2

$$
1
$$

0 
Table 3.-Benthic macrofauna data-Continued

\begin{tabular}{|c|c|c|c|c|c|}
\hline \multirow{3}{*}{ Taxonomic entry } & \multirow{3}{*}{$\begin{array}{l}\text { Kinnetic } \\
\text { Laboratory } \\
\text { code }\end{array}$} & \multicolumn{4}{|c|}{ Individuals } \\
\hline & & \multicolumn{4}{|c|}{ Replicate number } \\
\hline & & 1 & 3 & 4 & 5 \\
\hline
\end{tabular}

$\underline{\text { San Leandro }}$

Sampling datc: July 27, 1988 - Continued

\begin{tabular}{|c|c|}
\hline \multicolumn{2}{|c|}{ Crepidula so. A (Shrake) _... . . . 5570331} \\
\hline Exogone lourei & .4810066 \\
\hline Glycinde polygnatha & .4810496 \\
\hline Harmothoe imbricata & .4810343 \\
\hline Hydrozoa, unident. & .3710052 \\
\hline Leitoscoloplos pugettensis & .4810516 \\
\hline Marphysa sanguinea & .4810248 \\
\hline Musculista senhousia & .5540401 \\
\hline Nematodes, unident. & .4500001 \\
\hline Nephlys cornula franciscano & .4810116 \\
\hline Philine sp. (A) (SCAMIT) . & .5570240 \\
\hline Porifera & .3600000 \\
\hline Pyromaia tuberculata & .5286094 \\
\hline Sarsiella zostericola & .5220091 \\
\hline Schistomeringos rudolphi & .4810354 \\
\hline Sphaerosyllis californiensis & .4810272 \\
\hline Streblospio benedicti ... & .4810257 \\
\hline Tapes japonica & .5540158 \\
\hline
\end{tabular}

$\begin{array}{rrrrr}0 & 12 & 0 & 2 & 0 \\ 0 & 1 & 0 & 12 & 0 \\ 4 & 5 & 3 & 7 & 3 \\ 0 & 1 & 2 & 0 & 0 \\ 0 & 0 & 0 & 0 & 1 \\ 0 & 0 & 1 & 0 & 0 \\ 5 & 1 & 2 & 0 & 0 \\ 5 & 248 & 71 & 29 & 12 \\ 0 & 0 & 0 & 36 & 3 \\ 0 & 0 & 0 & 1 & 2 \\ 0 & 0 & 0 & 1 & 0 \\ 0 & 1 & 0 & 0 & 0 \\ 1 & 0 & 0 & 0 & 0 \\ 4 & 0 & 0 & 1 & 4 \\ 0 & 1 & 0 & 0 & 0 \\ 0 & 0 & 1 & 0 & 0 \\ 0 & 1 & 0 & 0 & 0 \\ 1 & 26 & 7 & 4 & 1\end{array}$

Sampling date: September 13, 1988

\begin{tabular}{|c|c|c|c|c|c|}
\hline Amaeana occidentalis ............4810001 & 0 & 1 & 0 & 0 & 0 \\
\hline Ampelisca abdita . . . . . . . . . . . . .5275504 & 302 & 236 & 297 & 718 & 239 \\
\hline$\ldots \ldots \ldots \ldots 4810565$ & 7 & 8 & 2 & 7 & 2 \\
\hline Campanularidae, unident. . ..........3710039 & 1 & 0 & 1 & 0 & 1 \\
\hline Capitellidae, unident. . ...........4810558 & 0 & 0 & 0 & 0 & 1 \\
\hline Cirratulidae, unident. .............4810990 & 1 & 0 & 1 & 1 & 2 \\
\hline Corophium sp. a ..............5275510 & 328 & 190 & 91 & 11 & 131 \\
\hline Cossura pygodactylata ...........4810861 & 0 & 0 & 2 & 2 & 1 \\
\hline Crepidula plana ..................... .5570204 & 8 & 8 & 5 & 10 & 6 \\
\hline Crepidula $\mathrm{sp(p)......} \mathrm{.} \mathrm{.} \mathrm{.} \mathrm{.} \mathrm{.} \mathrm{.} \mathrm{.} \mathrm{.} \mathrm{.} \mathrm{.} \mathrm{.} \mathrm{.} \mathrm{.} \mathrm{.5570203}$ & 3 & 5 & 5 & 0 & 4 \\
\hline Crepidula sp. A (Shrake) .............5570331 & 1 & 1 & 1 & 2 & 3 \\
\hline Cryptomya californica ............5540155 & 6 & 1 & 1 & 0 & 1 \\
\hline Euchone limnicola ...............4810255 & 12 & 20 & 21 & 6 & 14 \\
\hline Exogone lourei ...............4810066 & 29 & 21 & 21 & 26 & 13 \\
\hline Glycinde polygnatha ..............4810496 & 7 & 4 & 5 & 5 & 12 \\
\hline Grandidierella japonica ...........5275503 & 12 & 8 & 2 & 10 & 12 \\
\hline Harmothoe imbricata .............4810343 & 1 & 1 & 1 & 0 & 2 \\
\hline Hemigrapsus oregonensis . ..........5286092 & 1 & 0 & 0 & 0 & 0 \\
\hline Leitoscoloplos pugettensis . .........4810516 & 0 & 1 & 0 & 0 & 0 \\
\hline Leucon subnasica ...............5263012 & 0 & 0 & 0 & 1 & 0 \\
\hline Marphysa sanguinea . . . . . . . . . . .4810248 & 2 & 0 & 5 & 8 & 5 \\
\hline Mediomastus sp(p). . .............4810598 & 1 & 0 & 1 & 0 & 0 \\
\hline
\end{tabular}


Table 3.-Benthic macrofauna data-Continued

\begin{tabular}{lccccc}
\hline \multirow{2}{*}{ Taxonomic entry } & Kinnetic & \multicolumn{5}{c}{ Individuals } \\
\cline { 2 - 6 } & Laboratory & \multicolumn{4}{c}{ Replicate number } \\
\cline { 2 - 6 } & code & 1 & 2 & 3 & 4 \\
\hline
\end{tabular}

\section{$\underline{\text { San Leandro }}$}

Sampling date: September 13, 1988—Continued

Melanochlamys diomedea ...........5570248

Musculista senhousia ..............5540401

Nematodes, unident. .............4500001

Nephtys cornuta franciscana ..........4810116

Oligochaete, unident. ............4880001

Philine sp. (A) (SCAMIT) .......... 5570240

Phoronis $\mathrm{sp}(\mathrm{p}) . \ldots \ldots \ldots \ldots \ldots \ldots . \ldots 5700002$

Platyhelminthid, unident. ...........3900001

Polydora ligni ...................44810168

Porifera ....................... 3600000

Pyromaia tuberculata ..............5286094

Sarsiella zostericola ..............5220091

Schistomeringos rudolphi ...........4810354

Sphaerosyllis californiensis .........4810272

Tapes japonica ..................5540158

Theora lubrica ....................55540114

$\begin{array}{rr}0 & 0 \\ 1 & 1 \\ 224 & 0 \\ 1 & 2 \\ 38 & 11 \\ 0 & 0 \\ 0 & 2 \\ 5 & 0 \\ 15 & 6 \\ 0 & 0 \\ 0 & 2 \\ 26 & 7 \\ 5 & 4 \\ 7 & 0 \\ 11 & 6 \\ 40 & 32\end{array}$

$\begin{array}{rrr}0 & 0 & 2 \\ 0 & 18 & 0 \\ 256 & 18 & 43 \\ 4 & 6 & 0 \\ 37 & 14 & 51 \\ 0 & 1 & 0 \\ 1 & 0 & 0 \\ 2 & 0 & 3 \\ 0 & 19 & 4 \\ 0 & 1 & 0 \\ 0 & 3 & 1 \\ 16 & 13 & 19 \\ 6 & 8 & 4 \\ 2 & 1 & 2 \\ 2 & 10 & 11 \\ 18 & 23 & 16\end{array}$

Sampling date: November 2, 1988

Ampelisca abdita ...............55275504

Amphiuridae, unident. .............5930032

Armandia brevis ................44810012

Asychis elongata ...............4810565

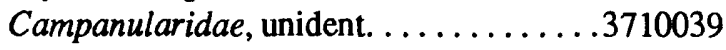

Cirratulidae, unident. ..............4810990

Corophium sp(p).................55275098

Corophium sp. a ...............5275510

Cossura pygodactylata ............4810861

Euchone limnicola ................4810255

Exogone lourei ................4810066

Glycinde polygnatha .............4810496

Harmothoe imbricata ..............4810343

Leitoscoloplos pugettensis .........4810516

Leucon subnasica ..............55263012

Marphysa sanguinea ............4810248

Melanochlamys diomedea ..........5570248

Musculista senhouisia ............5540401

Nematodes, unident. ............4500001

Nephtys cornuta franciscana .........4810116

Oligochaete, unident. ............4880001

Philine sp. (A) (SCAMIT) _..........5570240

Platyhelminthid, unident. ..........3900001

Polydora ligni .................4810168

$\begin{array}{rr}2,609 & 2,896 \\ 0 & 0 \\ 0 & 1 \\ 1 & 7 \\ 0 & 0 \\ 1 & 0 \\ 1 & 3 \\ 0 & 1 \\ 1 & 0 \\ 9 & 19 \\ 54 & 72 \\ 0 & 1 \\ 6 & 6 \\ 0 & 1 \\ 0 & 0 \\ 3 & 2 \\ 0 & 0 \\ 43 & 59 \\ 18 & 46 \\ 1 & 4 \\ 17 & 9 \\ 1 & 3 \\ 0 & 1 \\ 0 & 0\end{array}$

$\begin{array}{rr}2,556 & 2,091 \\ 1 & 0 \\ 0 & 0 \\ 6 & 11 \\ 1 & 0 \\ 0 & 0 \\ 0 & 1 \\ 0 & 12 \\ 2 & 0 \\ 10 & 10 \\ 81 & 23 \\ 1 & 0 \\ 2 & 6 \\ 0 & 1 \\ 1 & 0 \\ 3 & 4 \\ 1 & 0 \\ 49 & 16 \\ 186 & 149 \\ 1 & 0 \\ 12 & 19 \\ 1 & 2 \\ 0 & 0 \\ 2 & 0\end{array}$


Table 3.-Benthic macrofauna data-Continued

\begin{tabular}{lccccc}
\hline & Kinnetic & \multicolumn{5}{c}{ Individuals } \\
\cline { 2 - 6 } Taxonomic entry & Laboratory & \multicolumn{4}{c}{ Replicate number } \\
\cline { 3 - 6 } & code & 1 & 2 & 3 & 4 \\
\hline
\end{tabular}

$\underline{\text { San Leandro }}$

Sampling date: November 2, 1988-Continued

Pyromaia tuberculata ..............5286094

Sarsiella zostericola ...............5220091

Schistomeringos rudolphi ...........4810354

Sphaerosyllis californiensis ........4810272

Tapes japonica ..................5540158

Theora lubrica ....................55540114

$\begin{array}{rr}1 & 1 \\ 13 & 9 \\ 4 & 2 \\ 3 & 6 \\ 27 & 37 \\ 11 & 32\end{array}$

$\underline{\text { San Pablo Deep }}$

Sampling date: January 12,1988
Amaeana occidentalis ..............4810001

Ampelisca abdita .................. 5275504

Cirratulidae, unident. . . . . . . . . . . . . 4810990

Corophium alienense .............. 5275287

Gemma gemma .................55540400

Glycinde polygnatha ..............4810496

Leucon subnasica ...............55263012

Mediomastus sp. ...............4810303

Nematodes, unident. .............4500001

Odostomia (Evalea) sp. $N$ (Shrake) ....5570321

Oligochaete, unident. .............4880001

Platyhelminthid, unident. ...........3900001

Polydora brachycephala ............4810557

Potamocorbula amurensis ..........5540214

Pseudopolydora kempi ............4810640

Synidotea laticauda ...............55265110

Tapes japonica .................5540158

$\begin{array}{rr}0 & 0 \\ 2 & 0 \\ 0 & 1 \\ 0 & 0 \\ 3 & 0 \\ 1 & 1 \\ 2 & 0 \\ 1 & 0 \\ 2 & 0 \\ 0 & 0 \\ 5 & 1 \\ 0 & 0 \\ 0 & 0 \\ 79 & 93 \\ 0 & 0 \\ 0 & 0 \\ 0 & 0\end{array}$

Sampling date: March 15, 1988
Ampelisca abdita ................ 5275504

Asychis elongata ................4810565

Capitella capitata ..............4810241

Gemma gemma ................ 5540400

Glycinde polygnatha .............44810496

Nematodes, unident. . . . . . . . . . . . 4500001

Oligochaete, unident. .............44880001

Potamocorbula amurensis . . . . . . . . 5540214

Rhepoxynius $\mathrm{sp}(\mathrm{p})$. ..............5275241

Scolelepis squamata ..............4810589

Tapes japonica ................5540158

Tharyx sp. ...................4810595
$0 \quad 0$

$0 \quad 0$

$0 \quad 0$

$0 \quad 0$

11

30

$19 \quad 6$

$51 \quad 59$

10

$0 \quad 0$

0

1

$\begin{array}{rrr}0 & 3 & 7 \\ 10 & 6 & 10 \\ 2 & 0 & 3 \\ 10 & 2 & 2 \\ 30 & 15 & 15 \\ 18 & 23 & 57\end{array}$

\section{7}

10

3

15

57 
Table 3.-Benthic macrofauna data-Continued

\begin{tabular}{lcccccc}
\hline \multirow{2}{*}{ Taxonomic entry } & Kinnetic & \multicolumn{5}{c}{ Individuals } \\
\cline { 3 - 6 } & Laboratory & \multicolumn{5}{c}{ Replicate number } \\
\cline { 3 - 6 } & code & 1 & 2 & 3 & 4 & 5 \\
\hline
\end{tabular}

$\underline{\text { San Pablo Deep }}$

Sampling date: May 24, 1988

\begin{tabular}{|c|c|c|c|c|c|}
\hline Ampelisca abdita ............... & 0 & 0 & 8 & 1 & 0 \\
\hline Amphiurid, unident. . ..............5930014 & 0 & 1 & 0 & 0 & 0 \\
\hline Balanus crenatus ................ 5250036 & 0 & 1 & 23 & 96 & 15 \\
\hline Balanus $\mathrm{sp}(\mathrm{p}) . \ldots \ldots \ldots \ldots \ldots \ldots \ldots . \ldots \ldots 250002$ & 3 & 4 & 0 & 0 & 0 \\
\hline Cerebratulus $\mathrm{sp}(\mathrm{p}) . \ldots \ldots \ldots \ldots \ldots \ldots \ldots \ldots \ldots \ldots \ldots \ldots 14$ & 0 & 0 & 1 & 0 & 0 \\
\hline Corophium $\mathrm{sp}(\mathrm{p}) \ldots \ldots \ldots \ldots \ldots \ldots . . .5275098$ & 0 & 0 & 0 & 1 & 2 \\
\hline Corophium sp. $a$.................5275510 & 28 & 91 & 75 & 54 & 99 \\
\hline Cryptomya californica ............5540155 & 1 & 0 & 0 & 0 & 0 \\
\hline Exogone lourei .................4810066 & 0 & 1 & 0 & 0 & 0 \\
\hline Glycinde polygnatha ...............4810496 & 1 & 3 & 6 & 4 & 2 \\
\hline Grandidierella japonica .............5275503 & 0 & 1 & 0 & 1 & 0 \\
\hline Harmothoe imbricata ...............44810343 & 0 & 0 & 0 & 1 & 0 \\
\hline Hydrozoa, unident. . ............. 3710052 & 0 & 32 & 0 & 0 & 14 \\
\hline Musculista senhousia ..............5540401 & 0 & 0 & 0 & 0 & 1 \\
\hline Nemertea, unident. . . . . . . . . . . 4000002 & 2 & 0 & 0 & 0 & 0 \\
\hline Odostomia (Evalea) sp. H (Shrake) . . ...5570314 & 2 & 1 & 0 & 0 & 2 \\
\hline Oligochaete, unident. . . . . . . . . . . 48880001 & 0 & 0 & 1 & 1 & 0 \\
\hline Polydora brachycephala ............4810557 & 16 & 47 & 8 & 38 & 42 \\
\hline Polydora ligni ...................44810168 & 0 & 2 & 0 & 0 & 0 \\
\hline Potamocorbula amurensis ...........5540214 & 1 & 32 & 13 & 6 & 32 \\
\hline Sarsiella zostericola ..............5220091 & 1 & 1 & 1 & 4 & 4 \\
\hline Scleroplax granulata ..............5286519 & 0 & 0 & 0 & 1 & 0 \\
\hline Scolelepis $\mathrm{sp}(\mathrm{p}) \ldots \ldots \ldots \ldots \ldots \ldots \ldots . \ldots 4810316$ & 1 & 0 & 0 & 0 & 0 \\
\hline Stenothoid, unident. . . . . . . . . . . . . . 5275122 & 0 & 0 & 1 & 0 & 0 \\
\hline Upogebia pugettensis ..............5286103 & 0 & 0 & 1 & 0 & 0 \\
\hline
\end{tabular}

Sampling date: July 20,1988

\begin{tabular}{|c|c|c|c|c|c|}
\hline Ampelisca abdita & 0 & 0 & 1 & 0 & 0 \\
\hline Aporobopyrus muguensis . ..........5265041 & 0 & 0 & 2 & 0 & 0 \\
\hline Balanus crenatus ................5250036 & 0 & 0 & 0 & 0 & 22 \\
\hline Corophium sp. $a$ & 0 & 0 & 4 & 0 & 0 \\
\hline Glycinde polygnatha & 1 & 0 & 0 & 0 & 0 \\
\hline Leptochelia dubia & 0 & 0 & 0 & 0 & 1 \\
\hline Lineidae, unident. . .............4000038 & 0 & 0 & 0 & 2 & 0 \\
\hline Natantia, unident. . . . . . . . . . 5286111 & 1 & 0 & 0 & 0 & 0 \\
\hline Odostomia (Evalea) sp. H (Shrake) . ....5570314 & 0 & 0 & 1 & 0 & 0 \\
\hline Odostomia (Evalea) sp. J (Shrake) ......5570305 & 1 & 0 & 0 & 0 & 0 \\
\hline Odostomia (Evalea) sp. $N$ (Shrake) ....5570321 & 1 & 0 & 0 & 0 & 0 \\
\hline Philine sp. (A) (SCAMIT) ...........5570240 & 0 & 0 & 1 & 0 & 0 \\
\hline Phoronis sp(p). ...............5700002 & 0 & 0 & 10 & 0 & 0 \\
\hline Pleusymtes sp(p). . . . . . . . . . . . 5275203 & 0 & 0 & 0 & 0 & 1 \\
\hline Polydora brachycephala & 0 & 2 & 10 & 3 & 1 \\
\hline
\end{tabular}


Table 3.-Benthic macrofauna data-Continued

\begin{tabular}{lccccc}
\hline \multirow{2}{*}{ Taxonomic entry } & Kinnetic & \multicolumn{5}{c}{ Individuals } \\
\cline { 2 - 6 } & Laboratory & \multicolumn{4}{c}{ Replicate number } \\
\cline { 2 - 6 } & code & 1 & 2 & 3 & 4 \\
\hline
\end{tabular}

San Pablo Deep

Sampling date: July 20, 1988—Continued

Polamocorbula amurensis ..........5540214

Sarsiella zostericola ...............5220091

Upogebia pugettensis ............5286103

$\begin{array}{rr}30 & 21 \\ 0 & 0 \\ 0 & 0\end{array}$

Sampling date: September 13, 1988

$\begin{array}{rrrrr}34 & 15 & 42 & 44 & 39 \\ 0 & 0 & 2 & 1 & 2 \\ 0 & 0 & 1 & 0 & 7 \\ 0 & 0 & 1 & 0 & 0 \\ 0 & 0 & 1 & 0 & 0 \\ 0 & 0 & 2 & 0 & 0 \\ 104 & 71 & 122 & 111 & 148 \\ 5 & 6 & 2 & 4 & 2 \\ 0 & 0 & 0 & 0 & 1 \\ 0 & 0 & 2 & 1 & 2 \\ 1 & 0 & 0 & 0 & 0 \\ 0 & 1 & 0 & 0 & 0 \\ 0 & 0 & 1 & 0 & 0 \\ 1 & 0 & 6 & 4 & 0 \\ 0 & 0 & 5 & 0 & 1 \\ 0 & 0 & 1 & 0 & 0 \\ 1 & 1 & 9 & 0 & 18 \\ 0 & 1 & 0 & 0 & 1 \\ 15 & 29 & 8 & 1 & 7 \\ 0 & 0 & 18 & 0 & 2 \\ 11 & 2 & 17 & 10 & 19 \\ 6 & 0 & 3 & 0 & 2 \\ 1 & 0 & 1 & 1 & 0 \\ 0 & 0 & 0 & 0 & 1 \\ 0 & 0 & 0 & 1 & 0 \\ 0 & 0 & 1 & 0 & 0 \\ 0 & 1 & 0 & 0 & 0\end{array}$

Sampling date: November 1, 1988

Ampelisca abdita .................55275504

Balanus crenatus . . . . . . . . . . . . . . 5250036

Campanularidae, unident. .............3710039

Corophium sp(p). . . . . . . . . . . . . . . . . .5275098

Corophium sp. a .................5275510

Crangon $\mathrm{sp}(\mathrm{p}) . . . \ldots \ldots \ldots . . . . . . . .5286514$

Eusyllis transecta ...............4810680

$\begin{array}{ll}2 & 0 \\ 2 & \\ 1 & \\ 1 & \\ 0 & \\ 1 & \\ 0 & \end{array}$

$\begin{array}{rll}46 & 0 & 1 \\ 0 & 1 & 0 \\ 1 & 0 & 0\end{array}$

\begin{tabular}{|c|c|c|}
\hline$\ldots \ldots \ldots \ldots .5275504$ & 34 & 15 \\
\hline Asychis elongata ...............4810565 & 0 & 0 \\
\hline Balanus crenatus . . . . . . . . . . . ...5250036 & 0 & 0 \\
\hline Brachyura, unident. . . . . . . . . . . . . . . 5286098 & 0 & 0 \\
\hline Cerebratulus $\mathrm{sp}(\mathrm{p}) \ldots \ldots \ldots \ldots \ldots \ldots . . .4000014$ & 0 & 0 \\
\hline Cirratulidae, unident. .............4810990 & 0 & 0 \\
\hline Corophium sp. $a$...............5275510 & 104 & 71 \\
\hline Glycinde polygnatha ..............4810496 & 5 & 6 \\
\hline Grandidierella japonica . . . . . . . . . . 5275503 & 0 & 0 \\
\hline Heteromastus filiformis . . . . . . . . . . . . . 4810438 & 0 & 0 \\
\hline Lineidae, unident. . . . . . . . . . . . . .44000038 & 1 & 0 \\
\hline Molgula manhattensis .............66301075 & 0 & 1 \\
\hline Mysids, unident. . . . . . . . . . . . . . . .5262006 & 0 & 0 \\
\hline Nematodes, unident. . ............ & 1 & 0 \\
\hline Oligochaete, unident. . . . . . . . . . . . 48880001 & 0 & 0 \\
\hline Paleanotus bellis .................4810139 & 0 & 0 \\
\hline Phoronis sp(p). . ................5700002 & 1 & 1 \\
\hline Pleusymtes $\mathrm{sp}(\mathrm{p}) \ldots \ldots \ldots \ldots \ldots \ldots \ldots . . . .5275203$ & 0 & 1 \\
\hline Polydora brachycephala . . . . . . . . . . .4810557 & 15 & 29 \\
\hline Polydora ligni ...................4810168 & 0 & 0 \\
\hline Potamocorbula amurensis ...........5540214 & 11 & 2 \\
\hline Pyromaia tuberculata . . . . . . . . . . . 5286094 & 6 & 0 \\
\hline Sarsiella zostericola . . . . . . . . . . . 5220091 & 1 & 0 \\
\hline Synidotea laticauda . . ..............5265110 & 0 & 0 \\
\hline Tubulanus pellucidus ................4000011 & 0 & 0 \\
\hline Tubulanus $\mathrm{sp}(\mathrm{p}) . \ldots \ldots \ldots \ldots \ldots . . \ldots 4000013$ & 0 & 0 \\
\hline Upogebia pugettensis . . . . . . . . . . 5286103 & 0 & 1 \\
\hline
\end{tabular}

7

0$$
0
$$$$
8
$$

2 18 1 7 2 19 2 
Table 3.-Benthic macrofauna data-Continued

\begin{tabular}{lcccccc}
\hline \multirow{2}{*}{ Taxonomic entry } & Kinnetic & \multicolumn{5}{c}{ Individuals } \\
\cline { 2 - 6 } & Laboratory & \multicolumn{5}{c}{ Replicate number } \\
\cline { 2 - 6 } & code & 1 & 2 & 3 & 4 & 5 \\
\hline
\end{tabular}

$\underline{\text { San Pablo Deep }}$

Sampling date: November 1, 1988—Continued

Glycinde polygnatha ..............4810496

Harmothoe imbricata .............4810343

Hydrozoa, unident. ...............3710052

Molgula manhattensis ............66301075

Nematodes, unident. .............4500001

Odostomia (Evalea) sp. I (Shrake) ..... 5570317

Philine sp. (A) (SCAMIT) . . . . . . . . . 5570240

Phoronis $\mathrm{sp}(\mathrm{p})$. . . . . . . . . . . . . 5700002

Pleusymtes $\mathrm{sp}(\mathrm{p}) . \ldots \ldots \ldots \ldots \ldots \ldots . .5275203$

Polydora brachycephala ............44810557

Potamocorbula amurensis ..........5540214

Pyromaia tuberculata ............. 5286094

Scolelepis $\mathrm{sp}(\mathrm{p}) . \ldots \ldots \ldots \ldots \ldots \ldots . . . . .4810316$

Synidotea laticauda ...............55265110

Theora lubrica ...................5540114

$\begin{array}{rr}3 & 4 \\ 2 & 0 \\ 0 & 1 \\ 1 & 0 \\ 0 & 0 \\ 0 & 0 \\ 1 & 0 \\ 0 & 0 \\ 9 & 0 \\ 13 & 33 \\ 12 & 22 \\ 0 & 0 \\ 0 & 0 \\ 2 & 0 \\ 1 & 0\end{array}$

$\begin{array}{rr}2 & 0 \\ 0 & 0 \\ 0 & 0 \\ 0 & 0 \\ 0 & 0 \\ 0 & 0 \\ 0 & 0 \\ 0 & 0 \\ 0 & 1 \\ 11 & 6 \\ 18 & 17 \\ 0 & 0 \\ 1 & 0 \\ 0 & 2 \\ 0 & 0\end{array}$

San Pablo Shallow

Sampling date: January 12, 1988

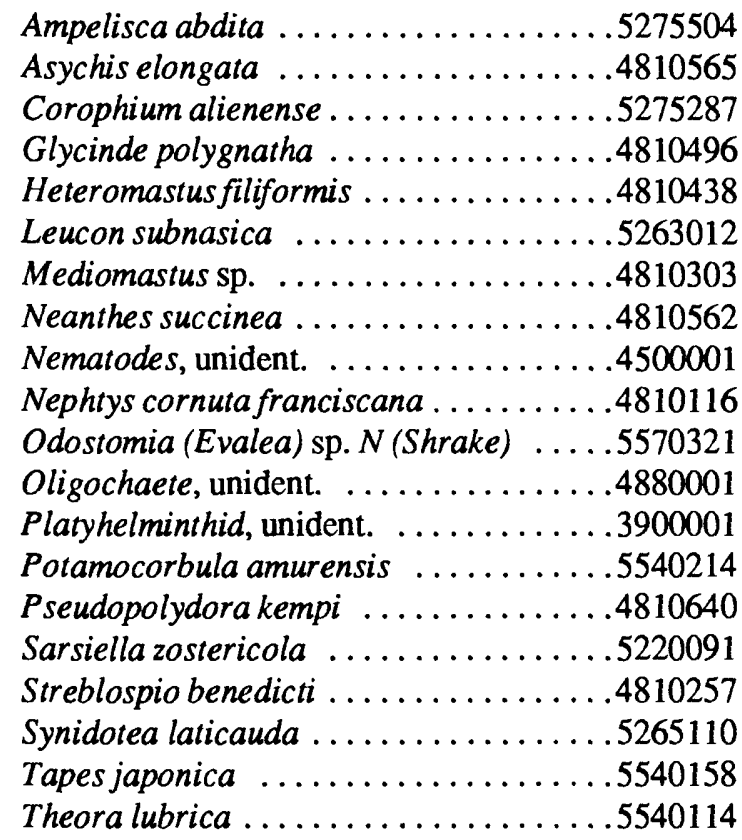

$\begin{array}{rrrrr}85 & 138 & 135 & 131 & 40 \\ 1 & 6 & 4 & 4 & 2 \\ 5 & 8 & 7 & 5 & 6 \\ 3 & 3 & 2 & 6 & 3 \\ 1 & 1 & 2 & 15 & 0 \\ 0 & 3 & 1 & 5 & 4 \\ 1 & 1 & 1 & 0 & 0 \\ 1 & 0 & 0 & 0 & 0 \\ 3 & 4 & 0 & 0 & 0 \\ 1 & 0 & 0 & 1 & 1 \\ 0 & 0 & 0 & 0 & 2 \\ 34 & 57 & 1 & 52 & 37 \\ 1 & 0 & 0 & 0 & 0 \\ 74 & 50 & 57 & 91 & 55 \\ 4 & 2 & 2 & 1 & 2 \\ 9 & 8 & 8 & 12 & 5 \\ 13 & 8 & 5 & 10 & 11 \\ 1 & 0 & 0 & 0 & 0 \\ 1 & 3 & 0 & 0 & 2 \\ 1 & 2 & 0 & 1 & 0\end{array}$


Table 3.-Benthic macrofauna data-Continued

\begin{tabular}{lcccccc}
\hline \multirow{2}{*}{ Taxonomic entry } & Kinnetic & \multicolumn{5}{c}{ Individuals } \\
\cline { 2 - 6 } & Laboratory & \multicolumn{5}{c}{ Replicate number } \\
\cline { 2 - 6 } & code & 1 & 2 & 3 & 4 & 5 \\
\hline
\end{tabular}

$\underline{\text { San Pablo Shallow }}$

Sampling date: March 14, 1988

Ampelisca abdita ................. 5275504

Asychis elongata ...............4810565

Campanularidae, unident. . . . . . . . . 3710039

Corophium alienense . . . . . . . . . . 5275287

Corophium sp(p)..................5275098

Cumella vulgaris .................5263098

Glycinde polygnatha ..............4810496

Leucon subnasica ................55263012

Macoma sp(p).................5540105

Molgula manhattensis ............66301075

Musculista senhousia ..............5540401

Nematodes, unident. ..............4500001

Nephtys cornuta franciscana ..........4810116

Odostomia (Evalea) sp. J (Shrake) ......5570305

Oligochaete, unident. . ...........4880001

Polynoid, unident. . ................4810936

Potamocorbula amurensis ..........5540214

Pseudopolydora kempi .............4810640

Sarsiella zostericola ...............5220091

Solen sicarius ...................5540416

Solenidae, unident. .................5540219

Streblospio benedicti . . . . . . . . . . . . . 48810257

Tapes japonica ...................55540158

Theora lubrica ..................... 5540114

Upogebia pugettensis .............5286103
118

0

1

2

9

2

84

0

0

1

4

0

0

50

0

36

3

9

2

0

16

4

4

0

104
12
0
6
24
2
3
14
1
0
0
10
1
0
89
0
52
0
7
0
0
5
3
1
0

427

163

7

0

14

44

10

0

32

0

0

0

3

0

1

41

0

57

0

15

0

1

13

6

2

Sampling date: May 31, 1988

Ampelisca abdita ...............55275504

Asychis elongata ................4810565

Capitella capitata ..............44810241

Corophium sp(p). . . . . . . . . . . . . . . . . 5275098

Corophium sp. $a$................55275510

Crangon nigricauda ..............5286120

Eudorella pacifica ...............55263112

Gemma gemma .................5540400

Glycinde polygnatha ..............4810496

Harmothoe imbricata ..............4810343

Harpacticoid copepod, unident. . ......5230052

Leitoscoloplos pugettensis ..........4810516

$\begin{array}{rr}791 & 1,013 \\ 4 & 8 \\ 1 & 2 \\ 5 & 8 \\ 18 & 25 \\ 0 & 0 \\ 1 & 1 \\ 0 & 0 \\ 7 & 11 \\ 0 & 0 \\ 0 & 0 \\ 0 & 0\end{array}$

545
2
0
9
19
1
0
1
11
2
0
0

$\begin{array}{rr}971 & 924 \\ 5 & 5 \\ 2 & 0 \\ 6 & 15 \\ 34 & 30 \\ 0 & 0 \\ 0 & 0 \\ 0 & 0 \\ 18 & 12 \\ 3 & 3 \\ 0 & 1 \\ 1 & 0\end{array}$


Table 3.-Benthic macrofauna data-Continued

\begin{tabular}{|c|c|c|c|c|c|}
\hline \multirow{3}{*}{ Taxonomic entry } & \multirow{3}{*}{$\begin{array}{c}\text { Kinnetic } \\
\text { Laboratory } \\
\text { code }\end{array}$} & \multicolumn{4}{|c|}{ Individuals } \\
\hline & & \multicolumn{4}{|c|}{ Replicate number } \\
\hline & & 1 & 3 & 4 & 5 \\
\hline
\end{tabular}

$\underline{\text { San Pablo Shallow }}$

Sampling date: May 31, 1988-Continued

Leucon subnasica ................5263012

Musculista senhousia .............5540401

Nematodes, unident. ..............4500001

Neomediomastus $\mathrm{sp}(\mathrm{p}) . \ldots \ldots \ldots \ldots . .4810865$

Nephtys cornuta franciscana ..........4810116

Odostomia (Evalea) sp.I (Shrake) ..... 5570317

Odostomia (Evalea) sp. N(Shrake) .... 5570321

Oligochaete, unident. .............4880001

Parapleustes derzhavini . . . . . . . . . 5275293

Philine sp. (A) (SCAMIT) ...........55570240

Potamocorbula amurensis . . ........55540214

Sarsiella zostericola ............... 5220091

Sphaerosyllis californiensis ........44810272

Streblospio benedicti ................4810257

Tapes japonica .................. 5540158

Theora lubrica .................... 5540114

Upogebia pugettensis ............5286103

$\begin{array}{rrrrr}8 & 17 & 14 & 16 & 6 \\ 0 & 0 & 2 & 2 & 1 \\ 6 & 27 & 4 & 25 & 1 \\ 0 & 0 & 0 & 1 & 0 \\ 0 & 1 & 0 & 1 & 1 \\ 0 & 0 & 1 & 0 & 0 \\ 0 & 0 & 0 & 0 & 1 \\ 15 & 15 & 0 & 9 & 12 \\ 0 & 0 & 0 & 2 & 0 \\ 1 & 0 & 0 & 0 & 0 \\ 447 & 491 & 355 & 457 & 526 \\ 18 & 18 & 7 & 6 & 8 \\ 1 & 0 & 0 & 0 & 0 \\ 1 & 2 & 0 & 0 & 0 \\ 1 & 8 & 0 & 3 & 2 \\ 0 & 0 & 5 & 5 & 0 \\ 0 & 0 & 0 & 2 & 1\end{array}$

Sampling date: July 25,1988

Ampelisca abdita .................. 5275504

Asychis elongata ...............4810565

Campanularidae, unident. ............ 3710039

Capitella capitata ................4810241

Corophium sp(p). . . . . . . . . . . . . . 5275098

Corophium sp. a ...............5275510

Ctenostomate, unident. ............5600114

Glycinde polygnatha ..............4810496

Grandidierella japonica ...........55275503

Harmothoe imbricata . . . . . . . . . . 4810343

Heteromastus filiformis . . . . . . . . . . . 4810438

Leitoscoloplos pugettensis . ........4810516

Leucon subnasica ................5263012

Melanochlamys diomedea . . . . . . . . . . 5570248

Musculista senhousia ..............5540401

Nematodes, unident. ................4500001

Nephtys cornuta franciscana . . . . . . . . .4810116

Odostomia (Evalea) sp. H (Shrake) . . . . 5570314

Oligochaete, unident. .............4880001

Pleusymtes $\mathrm{sp}(\mathrm{p}) \ldots \ldots \ldots \ldots \ldots \ldots . .5275203$

$\begin{array}{rr}208 & 276 \\ 4 & 6 \\ 0 & 1 \\ 0 & 0 \\ 3 & 2 \\ 10 & 10 \\ 0 & 1 \\ 7 & 11 \\ 0 & 0 \\ 1 & 0 \\ 0 & 3 \\ 1 & 0 \\ 0 & 5 \\ 0 & 0 \\ 3 & 0 \\ 0 & 4 \\ 0 & 0 \\ 1 & 0 \\ 0 & 5 \\ 1 & 0\end{array}$

$\begin{array}{rrr}159 & 191 & 278 \\ 4 & 7 & 5 \\ 0 & 0 & 0 \\ 1 & 1 & 0 \\ 4 & 1 & 4 \\ 7 & 9 & 6 \\ 0 & 0 & 0 \\ 7 & 13 & 11 \\ 0 & 1 & 0 \\ 0 & 0 & 0 \\ 0 & 0 & 1 \\ 0 & 1 & 0 \\ 1 & 1 & 4 \\ 1 & 0 & 0 \\ 2 & 1 & 2 \\ 0 & 0 & 0 \\ 0 & 4 & 2 \\ 1 & 1 & 3 \\ 0 & 0 & 0 \\ 0 & 0 & 0\end{array}$

40 BENTHIC MACROFAUNA AND ANCILLARY DATA, SAN FRANCISCO BAY, CALIFORNIA 
Table 3. - Benthic macrofauna data-Continued

\begin{tabular}{lcccccr}
\hline \multirow{2}{*}{ Taxonomic entry } & Kinnetic & \multicolumn{5}{c}{ Individuals } \\
\cline { 3 - 6 } & Laboratory & \multicolumn{5}{c}{ Replicate number } \\
\cline { 3 - 7 } & code & 1 & 2 & 3 & 4 & 5 \\
\hline
\end{tabular}

San Pablo Shallow

Sampling date: July 25,1988 - Continued

\begin{tabular}{|c|c|c|c|c|c|}
\hline Potamocorbula amurensis ..... & 378 & 263 & 238 & 459 & 499 \\
\hline Pseudopolydora kempi ............44810640 & 1 & 1 & 2 & 0 & 1 \\
\hline Sarsiella zostericola & 15 & 23 & 13 & 10 & 12 \\
\hline Sphaerosyllis californiensis .........4810272 & 0 & 1 & 0 & 0 & 0 \\
\hline Streblospio benedicti ...............4810257 & 0 & 2 & 0 & 1 & 0 \\
\hline Tapes japonica .................. & 1 & 7 & 3 & 1 & 1 \\
\hline Theora lubrica ....................5540114 & 1 & 1 & 0 & 1 & 2 \\
\hline
\end{tabular}

Sampling date: September 13, 1988

Ampelisca abdita ................5275504

Asychis elongata ................4810565

Bivalvia, unident. ................5540210

Campanularidae, unident. ............3710039

Capitella capitata ................4810241

Corophium sp. a ................5275510

Exogone lourei .................4810066

Glycinde polygnatha .............44810496

Harmothoe imbricata .............4810343

Hydrozoa, unident. .............. 3710052

Leitoscoloplos pugettensis .........4810516

Leucon subnasica ...............5263012

Lineidae, unident. ..............44000038

Mediomastus sp. ...............44810303

Musculista senhousia ..............5540401

Nematodes, unident. .............4500001

Nephtys caecoides ...............4810114

Nephtys cornuta franciscana ......... . 4810116

Odostomia (Evalea) sp. $H$ (Shrake) . ...5570314

Oligochaete, unident. .............4880001

Philine sp. (A) (SCAMIT) ........... 5570240

Pleusymtes $\mathrm{sp}(\mathrm{p})$. .................. 5275203

Potamocorbula amurensis ..........5540214

Pseudopolydora kempi ............4810640

Sarsiella zostericola ..............55220091

Sphaerosyllis californiensis ........4810272

Streblospio benedicti ...............4810257

Tapes japonica ................. 5540158

Theora lubrica ................... 5540114

$\begin{array}{rr}165 & 213 \\ 5 & 4 \\ 0 & 0 \\ 0 & 1 \\ 1 & 1 \\ 45 & 44 \\ 0 & 2 \\ 6 & 3 \\ 0 & 0 \\ 0 & 1 \\ 0 & 0 \\ 2 & 6 \\ 0 & 1 \\ 0 & 0 \\ 1 & 1 \\ 0 & 40 \\ 0 & 0 \\ 0 & 0 \\ 0 & 1 \\ 0 & 13 \\ 2 & 0 \\ 0 & 2 \\ 366 & 455 \\ 0 & 0 \\ 16 & 21 \\ 0 & 0 \\ 0 & 1 \\ 3 & 4 \\ 2 & 0\end{array}$

$\begin{array}{rrr}163 & 155 & 199 \\ 8 & 5 & 4 \\ 0 & 2 & 0 \\ 0 & 0 & 0 \\ 0 & 2 & 2 \\ 41 & 16 & 28 \\ 2 & 0 & 0 \\ 4 & 5 & 3 \\ 1 & 0 & 0 \\ 1 & 0 & 0 \\ 1 & 1 & 0 \\ 3 & 2 & 0 \\ 0 & 0 & 0 \\ 0 & 0 & 1 \\ 1 & 2 & 1 \\ 17 & 0 & 0 \\ 0 & 0 & 1 \\ 3 & 2 & 0 \\ 0 & 0 & 0 \\ 13 & 1 & 1 \\ 0 & 1 & 0 \\ 0 & 0 & 0 \\ 356 & 429 & 430 \\ 1 & 0 & 0 \\ 18 & 16 & 13 \\ 0 & 0 & 1 \\ 1 & 0 & 1 \\ 0 & 1 & 4 \\ 1 & 3 & 1\end{array}$


Table 3.-Benthic macrofauna data-Continued

\begin{tabular}{|c|c|c|c|c|c|}
\hline \multirow{3}{*}{ Taxonomic entry } & \multirow{3}{*}{$\begin{array}{c}\text { Kinnetic } \\
\text { Laboratory } \\
\text { code }\end{array}$} & \multicolumn{4}{|c|}{ Individuals } \\
\hline & & \multicolumn{4}{|c|}{ Replicate number } \\
\hline & & 1 & 3 & 4 & $\overline{5}$ \\
\hline
\end{tabular}

San Pablo Shallow

Sampling date: November 1,1988

Ampelisca abdita ................. 5275504

Asychis elongata ...............4810565

Campanularidae, unident. ...........3710039

Capitella capitata ...............4810241

Corophium sp. a ................ 5275510

Euchone limnicola ................4810255

Eudorella pacifica ................5263112

Exogone lourei .................4810066

Glycinde polygnatha ..............4810496

Heteromastus filiformis . . . . . . . . . . . 4810438

Hydrozoa, unident. ..............3710052

Leucon subnasica ................55263012

Magelona sacculata .............4810102

Mediomastus sp. ...............4810303

Natantia, unident. ..............5286111

Nematodes, unident. .............4500001

Nephtys caecoides ...............4810114

Nephtys cornuta franciscana ........4810116

Nephtys $\mathrm{sp}(\mathrm{p}) . \ldots \ldots \ldots \ldots \ldots \ldots \ldots . \ldots . \ldots . \ldots 10579$

Odostomia sp(p).................55570075

Oligochaete, unident. .............44880001

Philine sp. (A) (SCAMIT) ........... 5570240

Pleusymtes sp(p). .................55275203

Polydora ligni ..................4810168

Potamocorbula amurensis ..........5540214

Pyromaia tuberculata ............. 5286094

Sarsiella zostericola ..............5220091

Scolelepis squamata ..............44810589

Streblospio benedicti ..............4810257

Tapes japonica .................55540158

Theora lubrica ..................5540114

$\begin{array}{rrrrr}415 & 300 & 335 & 312 & 168 \\ 23 & 5 & 3 & 7 & 1 \\ 1 & 1 & 1 & 1 & 1 \\ 1 & 0 & 1 & 7 & 0 \\ 35 & 49 & 43 & 20 & 17 \\ 1 & 0 & 0 & 0 & 0 \\ 0 & 1 & 0 & 0 & 0 \\ 1 & 0 & 0 & 0 & 0 \\ 10 & 3 & 4 & 9 & 4 \\ 0 & 0 & 0 & 1 & 0 \\ 0 & 0 & 0 & 0 & 1 \\ 3 & 3 & 3 & 4 & 0 \\ 0 & 1 & 0 & 0 & 0 \\ 0 & 4 & 0 & 0 & 0 \\ 0 & 0 & 0 & 1 & 1 \\ 2 & 1 & 4 & 58 & 1 \\ 0 & 0 & 0 & 1 & 0 \\ 1 & 0 & 2 & 0 & 0 \\ 0 & 0 & 0 & 0 & 1 \\ 1 & 0 & 0 & 0 & 6 \\ 0 & 8 & 11 & 38 & 0 \\ 0 & 1 & 2 & 1 & 2 \\ 1 & 0 & 0 & 0 & 0 \\ 0 & 0 & 1 & 0 & 0 \\ 204 & 146 & 114 & 202 & 129 \\ 1 & 0 & 1 & 1 & 0 \\ 14 & 6 & 7 & 9 & 2 \\ 0 & 0 & 0 & 0 & 1 \\ 0 & 1 & 0 & 0 & 0 \\ 0 & 0 & 1 & 0 & 0 \\ 1 & 1 & 2 & 1 & 0\end{array}$

South Bay Deep

Sampling date: January 13,1988

Amaeana occidentalis .............4810001

Ampelisca abdita .................5275504

Amphipholis sp(p).................5930041

Amphiuridae, unident. . . . . . . . . . . 5930032

Anaitides longipes ...............4810505

Anthozoan, unident. ...............3730010

Asychis elongata

4810565

$\begin{array}{rr}1 & 1 \\ 4 & 2 \\ 0 & 1 \\ 1 & 0 \\ 0 & 0 \\ 100 & 32 \\ 21 & 14\end{array}$

1
2
1
0
0
32
14

$\begin{array}{rrr}0 & 2 & 2 \\ 3 & 2 & 16 \\ 0 & 2 & 0 \\ 1 & 1 & 0 \\ 1 & 0 & 0 \\ 221 & 92 & 6 \\ 18 & 16 & 20\end{array}$


Table 3.-Benthic macrofauna data-Continued

\begin{tabular}{|c|c|c|c|c|c|}
\hline \multirow{3}{*}{ Taxonomic entry } & \multirow{3}{*}{$\begin{array}{l}\text { Kinnetic } \\
\text { Laboratory } \\
\text { code }\end{array}$} & \multicolumn{4}{|c|}{ Individuals } \\
\hline & & \multicolumn{4}{|c|}{ Replicate number } \\
\hline & & 1 & 3 & 4 & 5 \\
\hline
\end{tabular}

South Bay Deep

Sampling date: January 13, 1988-Continued

Bivalvia, unident. . . . . . . . . . . . . . . . 5540210

Campanularidae, unident. ............3710039

Capitella capitata ..............44810241

Capitellidae, unident. ...........4810558

Caprella sp(p)..................55275117

Cerebratulus sp(p). ............... .4000014

Cirratulidae, unident. . . . . . . . . . . .4810990

Corophium alienense ..............5275287

Cossura pygodactylata ............44810861

Cryptomya californica .............5540155

Edwardsia sipunculoides ...........3730022

Epitonium sp...................5570117

Euchone limnicola ...............44810255

Eudorella pacifica ..............5263112

Eusyllis transecta ..............44810680

Exogone lourei ................44810066

Gemma gemma ................... 5540400

Glycinde polygnatha ..............4810496

Hemigrapsus oregonensis . . . . . . . . .5286092

Hesperonoe $\mathrm{sp}(\mathrm{p})$. . . . . . . . . . . . . . .44810090

Leptochelia dubia ................5264038

Mediomastus sp. . ...............4810303

Modiolus sp(p). ..................5540409

Molgula manhattensis . . . . . . . . . . . 66301075

Mya $\mathrm{sp}(\mathrm{p}) . \ldots \ldots \ldots \ldots \ldots \ldots \ldots . \ldots . \ldots 540124$

Mysella sp(p). .................55540137

Nematodes, unident. .............44500001

Neomediomastus $\mathrm{sp}(\mathrm{p}) . \ldots \ldots \ldots \ldots \ldots . \ldots 4810865$

Nephtys cornuta franciscana . . . . . . . . 4810116

Notomastus sp. .................. 4810389

Oligochaete, unident. . ............4880001

Paleanotus bellis .................4810139

Philine sp. (A) (SCAMIT) ...........5570240

Pleusymtes sp(p).................5275203

Polydora brachycephala ...........44810557

Polydora socialis ..................4810940

Pycnogonum stearnsi .............55202014

Sarsiella zostericola ..............5220091

Schistomeringos rudolphi ..........4810354

Sphaerosyllis bilobata ............44810833

Sphaerosyllis californiensis ........44810272

Tapes japonica ..................5540158

Tharyx sp.

$\begin{array}{rrrrr}1 & 0 & 0 & 0 & 0 \\ 0 & 1 & 1 & 0 & 1 \\ 2 & 1 & 5 & 3 & 1 \\ 1 & 0 & 0 & 0 & 2 \\ 1 & 0 & 0 & 0 & 0 \\ 3 & 0 & 1 & 1 & 0 \\ 0 & 0 & 0 & 1 & 0 \\ 2 & 3 & 0 & 1 & 16 \\ 4 & 4 & 5 & 4 & 0 \\ 0 & 1 & 1 & 0 & 0 \\ 0 & 0 & 1 & 0 & 0 \\ 2 & 0 & 1 & 1 & 0 \\ 0 & 0 & 0 & 0 & 3 \\ 0 & 2 & 0 & 1 & 0 \\ 0 & 1 & 0 & 0 & 0 \\ 70 & 183 & 77 & 180 & 27 \\ 0 & 2 & 0 & 5 & 0 \\ 6 & 11 & 10 & 8 & 3 \\ 0 & 0 & 1 & 0 & 0 \\ 1 & 0 & 0 & 0 & 0 \\ 11 & 3 & 13 & 15 & 1 \\ 15 & 19 & 12 & 10 & 7 \\ 1 & 0 & 0 & 2 & 0 \\ 0 & 2 & 0 & 1 & 0 \\ 0 & 0 & 0 & 0 & 1 \\ 0 & 0 & 0 & 1 & 0 \\ 3 & 6 & 3 & 34 & 1 \\ 0 & 0 & 0 & 2 & 0 \\ 6 & 4 & 3 & 2 & 1 \\ 0 & 0 & 1 & 0 & 1 \\ 2 & 9 & 16 & 26 & 3 \\ 0 & 0 & 1 & 0 & 0 \\ 0 & 0 & 1 & 0 & 0 \\ 2 & 0 & 1 & 0 & 0 \\ 0 & 0 & 0 & 0 & 1 \\ 1 & 0 & 0 & 0 & 0 \\ 0 & 0 & 0 & 1 & 0 \\ 7 & 6 & 6 & 8 & 6 \\ 2 & 0 & 2 & 2 & 3 \\ 0 & 1 & 1 & 0 & 0 \\ 8 & 4 & 6 & 2 & 0 \\ 0 & 4 & 0 & 5 & 1 \\ 0 & 2 & 0 & 0 & 0\end{array}$


Table 3.-Benthic macrofauna data-Continued

\begin{tabular}{lccccc}
\hline \multirow{2}{*}{ Taxonomic entry } & Kinnetic & \multicolumn{5}{c}{ Individuals } \\
\cline { 3 - 6 } & Laboratory & \multicolumn{4}{c}{ Replicate number } \\
\cline { 3 - 6 } & code & 1 & 2 & 3 & 4 \\
\hline
\end{tabular}

South Bay Deep

Sampling date: January 13,1988 - Continued

Theora lubrica ...................5540114

Tubulanus $\mathrm{sp}(\mathrm{p})$.
.4000013

Sampling date: March 8, 1988

\begin{tabular}{|c|c|c|c|c|c|}
\hline Amaeana occidentalis .............4810001 & 0 & 1 & 0 & 1 & 0 \\
\hline Ampelisca abdita ...............5275504 & 1 & 0 & 2 & 5 & 5 \\
\hline Amphiuridae, unident. .............5930032 & 0 & 0 & 0 & 1 & 0 \\
\hline Anaitides longipes . ..............4810505 & 0 & 0 & 1 & 0 & 0 \\
\hline Anthozoan, unident. . ............3730010 & 8 & 5 & 2 & 4 & 13 \\
\hline Asychis elongata ................4810565 & 46 & 16 & 35 & 32 & 25 \\
\hline Campanularidae, unident. . ..........3710039 & 1 & 1 & 1 & 0 & 1 \\
\hline Capitella capitata ................4810241 & 0 & 0 & 2 & 0 & 0 \\
\hline Cerebratulus $\mathrm{sp}(\mathrm{p}) . \ldots \ldots \ldots \ldots \ldots .4000014$ & 0 & 0 & 3 & 2 & 0 \\
\hline Cirriformia spirabrancha ...........4810854 & 0 & 1 & 0 & 0 & 0 \\
\hline Corophium alienense . ............55275287 & 1 & 3 & 2 & 0 & 1 \\
\hline Cossura pygodactylata ............4810861 & 6 & 12 & 6 & 14 & 4 \\
\hline Crepidula plana .................55570204 & 1 & 0 & 0 & 0 & 0 \\
\hline Cryptomya californica ............5540155 & 0 & 2 & 0 & 0 & 0 \\
\hline Ctenostomate, unident. ............5600114 & 0 & 0 & 0 & 0 & 1 \\
\hline Edwardsiidae, unident. ............3730045 & 0 & 1 & 0 & 1 & 0 \\
\hline Epitonium sp. .................5570117 & 0 & 1 & 0 & 0 & 0 \\
\hline Eudorella pacifica ...............5263112 & 1 & 0 & 1 & 1 & 0 \\
\hline Euphilomedes $\mathrm{sp}(\mathrm{p}) . \ldots \ldots \ldots \ldots \ldots . \ldots 5220069$ & 0 & 0 & 0 & 0 & 1 \\
\hline Exogone lourei ................4810066 & 158 & 147 & 229 & 226 & 136 \\
\hline Glycinde polygnatha ..............4810496 & 3 & 5 & 7 & 3 & 1 \\
\hline Hesperonoe $\mathrm{sp}(\mathrm{p}) . \ldots \ldots \ldots \ldots \ldots \ldots . \ldots . \ldots 10090$ & 0 & 0 & 0 & 1 & 0 \\
\hline Leptochelia dubia ...............55264038 & 11 & 6 & 10 & 2 & 3 \\
\hline Lineidae, unident. . ..............4000038 & 0 & 0 & 0 & 0 & 1 \\
\hline Mediomastus sp(p). . .............4810598 & 5 & 12 & 10 & 4 & 5 \\
\hline Modiolus sp(p). . ................5540409 & 0 & 1 & 1 & 0 & 0 \\
\hline Musculista senhousia .............55540401 & 2 & 0 & 0 & 1 & 0 \\
\hline Mysella sp(p). .................5540137 & 0 & 1 & 0 & 3 & 0 \\
\hline Nematodes, unident. . .............4500001 & 15 & 38 & 14 & 3 & 5 \\
\hline Nephtys cornuta franciscana ..........4810116 & 4 & 4 & 4 & 6 & 2 \\
\hline Notomastus sp. . ...............4810389 & 0 & 0 & 0 & 1 & 0 \\
\hline Oligochaete, unident. . . . . . . . . . . . . .44880001 & 2 & 1 & 1 & 2 & 0 \\
\hline Paraonidae, unident. .............4810708 & 0 & 0 & 0 & 1 & 0 \\
\hline Pettiboneia sanmatiensis . ...........4810552 & 0 & 0 & 0 & 0 & 4 \\
\hline Pholoe glabra .................4810442 & 0 & 0 & 0 & 1 & 0 \\
\hline Phoronis $\mathrm{sp}(\mathrm{p}) . \ldots \ldots \ldots \ldots \ldots \ldots \ldots 0002$ & 0 & 1 & 0 & 0 & 0 \\
\hline Porifera .....................3600000 & 0 & 1 & 0 & 0 & 0 \\
\hline Sarsiella zostericola .............5220091 & 9 & 14 & 8 & 9 & 11 \\
\hline
\end{tabular}


Table 3.-Benthic macrofauna data-Continued

\begin{tabular}{|c|c|c|c|}
\hline \multirow{2}{*}{ Taxonomic entry } & \multirow{3}{*}{$\begin{array}{c}\text { Kinnetic } \\
\text { Laboratory } \\
\text { code }\end{array}$} & \multicolumn{2}{|c|}{ Individuals } \\
\hline & & \multicolumn{2}{|c|}{ Replicate number } \\
\hline & & 1 & 3 \\
\hline
\end{tabular}

South Bay Deep

Sampling date: March 8, 1988—Continued

Schistomeringos rudolphi ..........44810354

Sphaerosyllis bilobata ..............4810833

Sphaerosyllis californiensis ........4810272

Tapes japonica .................5540158

Tharyx sp(p). . . . . . . . . . . . . . . 4810319

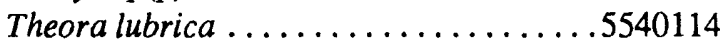

Transennella tantilla . . . . . . . . . . . . 5540189

Tubulanus $\mathrm{sp}(\mathrm{p}) . \ldots \ldots \ldots \ldots \ldots . . .4000013$

Upogebia pugettensis ............5286103

$\begin{array}{rrrrr}0 & 4 & 2 & 7 & 1 \\ 1 & 0 & 0 & 0 & 1 \\ 6 & 9 & 11 & 10 & 2 \\ 5 & 5 & 10 & 2 & 7 \\ 1 & 1 & 1 & 0 & 0 \\ 2 & 0 & 0 & 3 & 1 \\ 0 & 1 & 0 & 0 & 0 \\ 3 & 1 & 1 & 1 & 3 \\ 0 & 0 & 1 & 0 & 0\end{array}$

Sampling date: May 25, 1988

Ampelisca abdita .................5275504

Anaitides longipes ...............4810505

Anthozoan, unident. . . . . . . . . . . . . 3730010

Arabellid, unident. . . . . . . . . . . . .4810281

Asychis elongata ................4810565

Campanularidae, unident. ...........3710039

Cancer productus ................55286083

Cirriformia spirabrancha ............4810854

Corophium sp. a ................5275510

Cossura pygodactylata ............4810861

Crassostraea virginica .............55540195

Cryptomya californica .............5540155

Edwardsiidae, unident. ..............3730045

Eudorella pacifica ................55263112

Exogone lourei ..................4810066

Gastropoda, unident. ..............5570885

Glycinde polygnatha ...............4810496

Harmothoe imbricata . . . . . . . . . . . .4810343

Leptochelia dubia ................55264038

Leucon subnasica ...............5263012

Lineidae, unident. ...............4000038

Macoma sp(p)..................5540105

Mediomastus sp. ................4810303

Molgula manhattensis . . . . . . . . . . 6301075

Musculista senhousia ..............5540401

Nephtys cornuta franciscana . . . . . . . . .4810116

Oligochaete, unident. ..............4880001

Philine sp. (A) (SCAMIT) . . . . . . . . . . 5570240

Pholoides aspera . . . ................ 4810570

Phoronis sp(p). ..................55700002

Potamocorbula amurensis ...........5540214

$\begin{array}{rrrrr}424 & 290 & 300 & 378 & 115 \\ 0 & 1 & 0 & 0 & 2 \\ 1 & 2 & 0 & 11 & 6 \\ 0 & 1 & 0 & 0 & 0 \\ 47 & 54 & 55 & 16 & 15 \\ 0 & 0 & 1 & 1 & 0 \\ 0 & 0 & 0 & 0 & 1 \\ 0 & 2 & 0 & 1 & 1 \\ 319 & 218 & 135 & 31 & 42 \\ 1 & 0 & 0 & 0 & 0 \\ 0 & 0 & 0 & 1 & 0 \\ 0 & 1 & 0 & 0 & 0 \\ 1 & 0 & 0 & 0 & 0 \\ 3 & 2 & 4 & 4 & 6 \\ 54 & 43 & 54 & 116 & 26 \\ 0 & 0 & 0 & 1 & 0 \\ 10 & 8 & 7 & 8 & 4 \\ 0 & 0 & 0 & 0 & 1 \\ 3 & 4 & 5 & 7 & 16 \\ 0 & 1 & 0 & 1 & 1 \\ 1 & 0 & 0 & 0 & 0 \\ 0 & 1 & 0 & 0 & 0 \\ 0 & 2 & 2 & 3 & 0 \\ 0 & 0 & 0 & 0 & 1 \\ 4 & 6 & 0 & 2 & 1 \\ 3 & 11 & 5 & 7 & 3 \\ 3 & 1 & 0 & 0 & 0 \\ 4 & 1 & 4 & 0 & 3 \\ 0 & 0 & 0 & 1 & 0 \\ 0 & 0 & 0 & 2 & 0 \\ 2 & 0 & 0 & 0 & 0\end{array}$


Table 3. Benthic macrofauna data-Continued

\begin{tabular}{lcccccc}
\hline \multirow{2}{*}{ Taxonomic entry } & Kinnetic & \multicolumn{5}{c}{ Individuals } \\
\cline { 2 - 6 } & Laboratory & \multicolumn{4}{c}{ Replicate number } \\
\cline { 2 - 6 } & code & 1 & 2 & 3 & 4 & 5 \\
\hline
\end{tabular}

South Bay Deep

Sampling date: May 25, 1988-Continued

Pseudopolydora paucibranchiata ...... 4810347

Pycnogonida, unident. ............. 5202006

Pygodelphys aquilonaris ...........5230174

Pyromaia tuberculata ..............5286094

Rictaxis punctocaelatus .............5570083

Sabellaria sp(p). ...............4810190

Sarsiella zostericola .............5220091

Schistomeringos rudolphi ...........4810354

Scolelepis $\mathrm{sp}(\mathrm{p}) . \ldots \ldots \ldots \ldots \ldots \ldots \ldots . \ldots 4810316$

Sphaerosyllis bilobata ..............4810833

Sphaerosyllis californiensis ........4810272

Tapes japonica .................5540158

Theora lubrica .....................5540114

Tubulanus sp(p). ................4000013

Upogebia pugettensis ............5286103

$\begin{array}{rrrrr}0 & 0 & 0 & 1 & 0 \\ 0 & 0 & 0 & 0 & 2 \\ 0 & 0 & 0 & 0 & 1 \\ 0 & 1 & 1 & 1 & 0 \\ 0 & 1 & 0 & 0 & 0 \\ 0 & 0 & 0 & 1 & 0 \\ 12 & 7 & 7 & 11 & 0 \\ 0 & 3 & 1 & 3 & 2 \\ 0 & 0 & 1 & 0 & 0 \\ 2 & 0 & 0 & 1 & 0 \\ 3 & 4 & 0 & 3 & 0 \\ 13 & 13 & 22 & 20 & 15 \\ 0 & 1 & 1 & 0 & 0 \\ 0 & 0 & 1 & 0 & 0 \\ 0 & 0 & 1 & 0 & 1\end{array}$

Sampling date: July 25,1988

Acmira lopezi lopezi ..............4810853

Amaeana occidentalis .............44810001

Ampelisca abdita ................. 5275504

Amphipholis sp(p)...............5930041

Amphiuridae, unident. . . . . . . . . . . 5930032

Anthozoan, unident. .............. 3730010

Arabellid, unident. ...............4810281

Ascidiacea, unident. . . . . . . . . . .6.6301008

Asychis elongata .................4810565

Balanus crenatus ................5250036

Bivalvia, unident. . ................5540210

Campanularidae, unident. ............ 3710039

Capitella capitata ................4810241

Cerebratulus sp(p). ...............4000014

Cheilostomata unident. .............5600177

Cirriformia spirabrancha ...........44810854

Corophium sp(p).................55275098

Corophium sp. a ............... 5275510

Euchone limnicola ...............4810255

Eudorella pacifica ................55263112

Eusyllis transecta ..............4810680

Exogone lourei ................4810066

Gemma gemma .................5540400

Glycera $\operatorname{sp}(\mathrm{p}) . \ldots \ldots \ldots \ldots \ldots \ldots \ldots . . .4810079$

Glycinde polygnatha ............4810496

$\begin{array}{rrrrr}0 & 0 & 0 & 1 & 0 \\ 1 & 0 & 1 & 1 & 1 \\ 30 & 14 & 31 & 4 & 36 \\ 0 & 0 & 0 & 0 & 1 \\ 0 & 0 & 0 & 0 & 1 \\ 4 & 1 & 0 & 6 & 2 \\ 0 & 0 & 0 & 0 & 1 \\ 1 & 0 & 0 & 0 & 0 \\ 5 & 35 & 12 & 21 & 41 \\ 3 & 1 & 0 & 0 & 1 \\ 1 & 0 & 0 & 0 & 0 \\ 1 & 1 & 1 & 0 & 1 \\ 0 & 0 & 0 & 0 & 1 \\ 0 & 0 & 0 & 0 & 3 \\ 1 & 1 & 1 & 0 & 0 \\ 0 & 1 & 1 & 0 & 0 \\ 1 & 2 & 1 & 1 & 1 \\ 111 & 79 & 34 & 27 & 137 \\ 0 & 1 & 1 & 0 & 1 \\ 0 & 0 & 0 & 1 & 0 \\ 1 & 0 & 0 & 1 & 1 \\ 10 & 6 & 1 & 1 & 15 \\ 0 & 0 & 0 & 4 & 0 \\ 0 & 1 & 0 & 0 & 0 \\ 2 & 0 & 2 & 1 & 2\end{array}$


Table 3.-Benthic macrofauna data-Continued

\begin{tabular}{|c|c|c|c|c|c|c|}
\hline \multirow{2}{*}{ Taxonomic entry } & \multirow{3}{*}{$\begin{array}{c}\text { Kinnetic } \\
\text { Laboratory } \\
\text { code }\end{array}$} & \multicolumn{5}{|c|}{ Individuals } \\
\hline & & \multicolumn{5}{|c|}{ Replicate number } \\
\hline & & 1 & 2 & 3 & 4 & $\overline{5}$ \\
\hline
\end{tabular}

South Bay Deep

Sampling date: July $25,1988-$ Continued

\begin{tabular}{|c|c|c|c|c|}
\hline Harmothoe imbricata ............4810343 & 1 & 0 & 0 & 1 \\
\hline Heteromastus filiformis .............4810438 & 0 & 4 & 0 & 1 \\
\hline Hydrozoa, unident. . ............3710052 & 1 & 1 & 1 & 1 \\
\hline Leptochelia dubia ...............5264038 & 14 & 4 & 2 & 6 \\
\hline Leucon subnasica ................5263012 & 0 & 0 & 0 & 0 \\
\hline Lineidae, unident. . ..............4000038 & 0 & 0 & 2 & 1 \\
\hline Molgula manhattensis . ............6301075 & 0 & 0 & 0 & 5 \\
\hline Musculista senhousia .............5540401 & 1 & 0 & 0 & 6 \\
\hline Mysella sp. (A) (SCAMIT) . .......... 5540212 & 0 & 0 & 0 & 1 \\
\hline Nematodes, unident. . ............4500001 & 0 & 0 & 0 & 0 \\
\hline Nemertea, unident. . ............4000002 & 0 & 0 & 0 & 3 \\
\hline Neomediomastus sp(p). . ...........4810865 & 0 & 0 & 1 & 0 \\
\hline Nephtys cornuta franciscana . . . . . . . .4810116 & 1 & 1 & 1 & 3 \\
\hline Nephtys ferruginea ..............4810706 & 0 & 0 & 0 & 0 \\
\hline Philine sp. (A) (SCAMIT) . . . . . . . . 5570240 & 2 & 3 & 1 & 1 \\
\hline$\ldots \ldots \ldots \ldots \ldots .4810442$ & 0 & 1 & 0 & 0 \\
\hline Phoronis $\mathrm{sp}(\mathrm{p}) . . \ldots \ldots \ldots \ldots \ldots \ldots . \ldots . \ldots 00002$ & 0 & 0 & 0 & 1 \\
\hline Polydora sp(p). . ................4810588 & 1 & 0 & 0 & 0 \\
\hline Porifera .....................3600000 & 0 & 0 & 0 & 1 \\
\hline Pycnogonum stearnsi .............5202014 & 0 & 0 & 0 & 1 \\
\hline Pyromaia tuberculata .............55286094 & 0 & 1 & 0 & 0 \\
\hline Sarsiella zostericola .............5220091 & 11 & 1 & 7 & 1 \\
\hline Schistomeringos rudolphi ...........4810354 & 1 & 0 & 2 & 4 \\
\hline Sphaerosyllis bilobata ............4810833 & 0 & 0 & 0 & 0 \\
\hline Sphaerosyllis californiensis ........4810272 & 0 & 0 & 0 & 0 \\
\hline Synidotea laticauda ..............5265110 & 0 & 0 & 0 & 1 \\
\hline Tapes japonica ...............5540158 & 3 & 3 & 3 & 6 \\
\hline Theora lubrica ...................5540114 & 0 & 0 & 1 & 0 \\
\hline Tubulanus sp(p). . ..............4000013 & 0 & 0 & 0 & 0 \\
\hline
\end{tabular}

Sampling date: September 14, 1988

Amaeana occidentalis .............4810001

Ampelisca abdita ................55275504

Amphipholis sp(p)..................5930041

Anthozoan, unident. . . . . . . . . . . . . . 3730010

Armandia brevis ................4810012

Asychis elongata ................4810565

Autolytus sp(p). . . ...............4810277

Balanus crenatus ..................5250036

Brachyuran zoea ................55286063

Campanularidae, unident. ............3710039

Capitella capitata

$\begin{array}{rr}0 & 0 \\ 9 & 3 \\ 0 & 0 \\ 5 & 4 \\ 0 & 0 \\ 25 & 11 \\ 3 & 3 \\ 3 & 0 \\ 0 & 1 \\ 1 & 1 \\ 0 & 2\end{array}$

$\begin{array}{rrr}0 & 1 & 0 \\ 3 & 8 & 7 \\ 0 & 1 & 0 \\ 10 & 12 & 22 \\ 0 & 1 & 0 \\ 20 & 26 & 22 \\ 5 & 18 & 11 \\ 0 & 0 & 0 \\ 0 & 0 & 0 \\ 1 & 1 & 1 \\ 0 & 0 & 1\end{array}$


Table 3.-Benthic macrofauna data-Continued

\begin{tabular}{lcccccc}
\hline \multirow{2}{*}{ Taxonomic entry } & Kinnetic & \multicolumn{5}{c}{ Individuals } \\
\cline { 2 - 6 } & Laboratory & \multicolumn{5}{c}{ Replicate number } \\
\cline { 2 - 6 } & code & 1 & 2 & 3 & 4 & 5 \\
\hline
\end{tabular}

South Bay Deep

Sampling date: September 14, 1988-Continued

\begin{tabular}{|c|c|c|c|c|c|}
\hline Capitellidae, unident. .............4810558 & 0 & 0 & 0 & 0 & 1 \\
\hline Cerebratulus $\mathrm{sp}(\mathrm{p}) . \ldots \ldots \ldots \ldots \ldots . . . .4000014$ & 0 & 0 & 2 & 0 & 1 \\
\hline Cirratulidae, unident. . . . . . . . . . . . . 4810990 & 0 & 0 & 0 & 1 & 0 \\
\hline Corophium sp. a ................ 5275510 & 0 & 1 & 0 & 0 & 0 \\
\hline Cossura pygodactylata ............4810861 & 2 & 2 & 3 & 0 & 0 \\
\hline Crepidula $\mathrm{sp(p)...............55570203}$ & 0 & 0 & 1 & 0 & 0 \\
\hline Dorvilleidae, unident. . ..............4810583 & 3 & 3 & 0 & 0 & 0 \\
\hline Edwardsiidae, unident. . .............3730045 & 0 & 0 & 0 & 0 & 1 \\
\hline Eudorella pacifica ...............5263112 & 0 & 0 & 0 & 0 & 1 \\
\hline Eusyllis transecta ...............4810680 & 0 & 0 & 1 & 0 & 1 \\
\hline Exogone lourei .................4810066 & 50 & 55 & 77 & 60 & 55 \\
\hline Glycera robusta ...................4810532 & 0 & 0 & 0 & 1 & 0 \\
\hline Glycinde polygnatha ...............4810496 & 6 & 3 & 6 & 3 & 7 \\
\hline Granulina margaritula .............5570042 & 1 & 0 & 0 & 0 & 0 \\
\hline Harmothoe imbricata . . . . . . . . . . . 4810343 & 2 & 2 & 2 & 0 & 0 \\
\hline Heteromastus filiformis . . . . . . . . . . . 4810438 & 0 & 2 & 0 & 0 & 0 \\
\hline Hydrozoa, unident. . .............3710052 & 1 & 1 & 1 & 1 & 1 \\
\hline Leitoscoloplos pugettensis ..........4810516 & 0 & 0 & 0 & 0 & 1 \\
\hline Leptochelia dubia ..................5264038 & 26 & 13 & 20 & 21 & 26 \\
\hline Leucon subnasica ..................55263012 & 0 & 0 & 0 & 0 & 1 \\
\hline Mediomastus $\mathrm{sp}(\mathrm{p}) . \ldots \ldots \ldots \ldots \ldots \ldots . . \ldots 4810598$ & 2 & 0 & 3 & 4 & 0 \\
\hline Modiolus sp(p). ...................5540409 & 0 & 0 & 0 & 0 & 1 \\
\hline Molgula manhattensis . . . . . . . . . . .66301075 & 13 & 1 & 22 & 5 & 11 \\
\hline Musculista senhousia ...........5550401 & 5 & 0 & 0 & 1 & 1 \\
\hline Mytilus edulis ....................5540024 & 0 & 1 & 0 & 0 & 0 \\
\hline Neanthes succinea ............... 4810562 & 0 & 0 & 0 & 2 & 0 \\
\hline Nematodes, unident. . . . . . . . . . . . . 4500001 & 0 & 12 & 20 & 2 & 19 \\
\hline Nephtys cornuta franciscana . . . . . . . . . 4810116 & 3 & 1 & 3 & 1 & 1 \\
\hline Nudibranchia, unident. . . . . . . . . . . 5570976 & 0 & 0 & 1 & 0 & 0 \\
\hline Odostomia (Evalea) sp. H (Shrake) . . . .5570314 & 0 & 0 & 0 & 0 & 1 \\
\hline Oligochaete, unident. .............4880001 & 3 & 2 & 3 & 1 & 1 \\
\hline Philine sp. (A) (SCAMIT) . . . . . . . 5570240 & 0 & 1 & 3 & 5 & 0 \\
\hline Pholoides aspera ................4810570 & 0 & 0 & 0 & 0 & 2 \\
\hline Phoronis sp(p). ................55700002 & 0 & 3 & 0 & 1 & 0 \\
\hline Platyhelminthid, unident. ............3900001 & 1 & 0 & 0 & 0 & 0 \\
\hline Pleusymtes $\mathrm{sp}(\mathrm{p}) \ldots \ldots \ldots \ldots \ldots \ldots \ldots . . .5275203$ & 0 & 1 & 0 & 2 & 1 \\
\hline Polydora brachycephala ............4810557 & 0 & 1 & 0 & 0 & 0 \\
\hline Polydora ligni ..................4810168 & 4 & 3 & 1 & 0 & 0 \\
\hline Potamocorbula amurensis . . . . . . . . 5540214 & 0 & 0 & 0 & 0 & 1 \\
\hline Proceraea $\mathrm{sp}(\mathrm{p}) . \ldots \ldots \ldots \ldots \ldots \ldots . . .4810713$ & 8 & 0 & 3 & 8 & 10 \\
\hline Pseudopolydora paucibranchiata . . . . . . 4810347 & 1 & 0 & 2 & 0 & 0 \\
\hline Pyromaia tuberculata ..............5286094 & 1 & 2 & 4 & 0 & 3 \\
\hline Sarsiella zostericola ...............5220091 & 1 & 1 & 4 & 0 & 7 \\
\hline
\end{tabular}


Table 3.-Benthic macrofauna data-Continued

\begin{tabular}{|c|c|c|c|c|c|}
\hline \multirow{3}{*}{ Taxonomic entry } & \multirow{3}{*}{$\begin{array}{c}\text { Kinnetic } \\
\text { Laboratory } \\
\text { code }\end{array}$} & \multicolumn{4}{|c|}{ Individuals } \\
\hline & & \multicolumn{4}{|c|}{ Replicate number } \\
\hline & & 1 & 3 & 4 & 5 \\
\hline
\end{tabular}

South Bay Deep

Sampling date: September 14, 1988 -Continued

Schistocomus sp. A ...............4810892

Schistomeringos rudolphi ...........4810354

Sphaerosyllis californiensis ........44810272

Tapes japonica .................5540158

Theora lubrica ...................5540114

Thysanocardia nigra .............4000039

Tubulanus pellucidus..............4000011

$\begin{array}{lllll}0 & 0 & 0 & 1 & 0 \\ 0 & 2 & 0 & 1 & 3 \\ 9 & 8 & 8 & 2 & 5 \\ 6 & 4 & 4 & 6 & 2 \\ 0 & 1 & 5 & 2 & 1 \\ 0 & 1 & 0 & 0 & 0 \\ 1 & 2 & 1 & 0 & 2\end{array}$

Sampling date: November 1, 1988
Acmira catherinae ...............4810671

Amaeana occidentalis ..............4810001

Ampelisca abdita .................55275504

Amphipholis sp(p)................5930041

Amphiuridae, unident. .............5930032

Anthozoan, unident. . . . . . . . . . . . 3730010

Asychis elongata ................4810565

Autolytus sp(p). .................4810277

Brachyuran zoea ................5286063

Campanularidae, unident. ............3710039

Capitella capitata ................4810241

Cerebratulus sp(p). ...............4000014

Cirratulidae, unident. ............. 4810990

Cirriformia spirabrancha ...........4810854

Corophium sp. a ..............55275510

Cossura pygodactylata ............4810861

Crepidula sp(p)..................5570203

Cryptomya californica ............5540155

Dorvilleidae, unident. ............44810583

Epitonium sp. ....................5550117

Euchone limnicola ............... 4810255

Eudorella pacifica ................. 5263112

Eusyllis transecta ................4810680

Exogone lourei ................44810066

Glycera $\mathrm{sp}(\mathrm{p}) . \ldots \ldots \ldots \ldots \ldots . . . . .4810079$

Glycinde polygnatha ..............4810496

Harmothoe imbricata .............4810343

Harpacticoid copepod, unident. . . . . . . 5230052

Hemigrapsus oregonensis . . . . . . . . . . 5286092

Leitoscoloplos pugettensis .........4810516

Leptochelia dubia ...............55264038

Lineidae, unident. ...............4000038

Macoma sp(p)..................5540105

$\begin{array}{rrrrr}0 & 0 & 0 & 1 & 0 \\ 0 & 0 & 0 & 1 & 1 \\ 0 & 1 & 1 & 4 & 4 \\ 1 & 1 & 2 & 0 & 0 \\ 0 & 1 & 0 & 1 & 0 \\ 37 & 52 & 21 & 6 & 9 \\ 30 & 29 & 51 & 54 & 29 \\ 1 & 0 & 0 & 0 & 0 \\ 0 & 1 & 0 & 0 & 0 \\ 1 & 1 & 1 & 1 & 1 \\ 0 & 0 & 2 & 1 & 0 \\ 0 & 0 & 2 & 0 & 0 \\ 4 & 0 & 0 & 0 & 0 \\ 0 & 0 & 1 & 0 & 1 \\ 0 & 1 & 8 & 0 & 0 \\ 1 & 0 & 0 & 0 & 1 \\ 0 & 2 & 1 & 0 & 0 \\ 4 & 2 & 40 & 1 & 0 \\ 0 & 0 & 1 & 0 & 0 \\ 1 & 0 & 1 & 0 & 1 \\ 1 & 0 & 0 & 0 & 0 \\ 0 & 0 & 1 & 0 & 0 \\ 0 & 2 & 1 & 0 & 3 \\ 92 & 124 & 93 & 36 & 24 \\ 0 & 1 & 0 & 2 & 1 \\ 5 & 1 & 4 & 1 & 3 \\ 1 & 1 & 1 & 2 & 3 \\ 0 & 2 & 1 & 0 & 0 \\ 0 & 0 & 0 & 0 & 1 \\ 1 & 0 & 0 & 0 & 0 \\ 38 & 33 & 19 & 2 & 12 \\ 0 & 0 & 0 & 0 & 1 \\ 1 & 0 & 4 & 0 & 0\end{array}$


Table 3.-Benthic macrofauna data-Continued

\begin{tabular}{lccccc}
\hline & Kinnetic & \multicolumn{5}{c}{ Individuals } \\
\cline { 2 - 6 } Taxonomic entry & Laboratory & \multicolumn{4}{c}{ Replicate number } \\
\cline { 2 - 6 } & code & 1 & 2 & 3 & 4 \\
\hline
\end{tabular}

South Bay Deep

Sampling date: November 1, 1988-Continued

\begin{tabular}{|c|c|c|c|c|c|}
\hline$\ldots \ldots \ldots .4810303$ & 0 & 1 & 0 & 2 & 3 \\
\hline 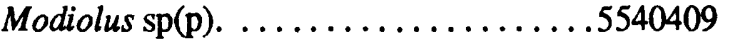 & 0 & 0 & 1 & 0 & 0 \\
\hline Molgula manhattensis .............6301075 & 105 & 155 & 106 & 28 & 30 \\
\hline Musculista senhousia .............5540401 & 29 & 113 & 80 & 6 & 11 \\
\hline Mysella sp. (A) (SCAMIT) . . . . . . . . . . 5540212 & 0 & 0 & 1 & 0 & 2 \\
\hline Mytilus edulis . . . . . . . . . . . . . ...5540024 & 0 & 0 & 3 & 0 & 0 \\
\hline Natantia, unident. . . . . . . . . . . . ...52861111 & 0 & 0 & 0 & 1 & 1 \\
\hline Nematodes, unident. ..............4500001 & 12 & 89 & 104 & 9 & 7 \\
\hline Nephtys cornuta franciscana . . . . . . . 4810116 & 8 & 9 & 15 & 6 & 6 \\
\hline Nuculana oxia ..................5540197 & 0 & 0 & 1 & 0 & 0 \\
\hline Oligochaete, unident. . ...........4880001 & 5 & 6 & 0 & 2 & 0 \\
\hline Philine sp. (A) (SCAMIT) . . . . . . . . . . 5570240 & 5 & 6 & 0 & 2 & 1 \\
\hline Pholoe glabra .................4810442 & 1 & 0 & 0 & 0 & 1 \\
\hline Phoronis sp(p). ................5700002 & 0 & 0 & 10 & 0 & 0 \\
\hline Platyhelminthid, unident. .......... 3900001 & 6 & 2 & 2 & 0 & 0 \\
\hline Polydora brachycephala ............4810557 & 1 & 0 & 0 & 0 & 0 \\
\hline Polydora ligni .................4810168 & 0 & 0 & 0 & 1 & 0 \\
\hline Polydora socialis . . . . . . . . . . . . . 4810940 & 0 & 0 & 0 & 1 & 0 \\
\hline Potamocorbula amurensis ..........5540214 & 0 & 1 & 0 & 0 & 0 \\
\hline Proceraea $\mathrm{sp}(\mathrm{p}) . \ldots \ldots \ldots \ldots \ldots \ldots \ldots 4810713$ & 2 & 0 & 0 & 0 & 0 \\
\hline Pygodelphys aquilonaris . ..........55230174 & 1 & 0 & 1 & 0 & 0 \\
\hline Pyromaia tuberculata .............55286094 & 1 & 1 & 1 & 1 & 6 \\
\hline Sarsiella zostericola .............55220091 & 2 & 4 & 0 & 0 & 4 \\
\hline Schistomeringos rudolphi ..........4810354 & 0 & 0 & 4 & 0 & 0 \\
\hline Sphaerosyllis bilobata .............4810833 & 0 & 1 & 0 & 0 & 0 \\
\hline Sphaerosyllis californiensis ........4810272 & 10 & 5 & 8 & 3 & 3 \\
\hline Tapes japonica ................5540158 & 5 & 7 & 6 & 2 & 2 \\
\hline Theora lubrica .................5540114 & 6 & 10 & 24 & 4 & 14 \\
\hline Thysanocardia nigra .............4000039 & 1 & 0 & 1 & 0 & 0 \\
\hline Tubulanus sp(p). ...............44000013 & 8 & 4 & 8 & 4 & 9 \\
\hline Upogebia pugettensis ............5286103 & 0 & 0 & 1 & 0 & 0 \\
\hline
\end{tabular}


Table 4.-Summary of benthic macrofauna data

\begin{tabular}{|c|c|c|c|}
\hline $\begin{array}{c}\text { Replicate } \\
\text { No. }\end{array}$ & $\begin{array}{c}\text { Taxonomic } \\
\text { entries }\end{array}$ & $\begin{array}{l}\text { Species } \\
\text { identified }\end{array}$ & Individuals \\
\hline \multicolumn{4}{|c|}{ Berkeley } \\
\hline \multicolumn{4}{|c|}{ Sampling date: January 12,1988} \\
\hline $\begin{array}{l}1 \\
2 \\
3 \\
4 \\
5\end{array}$ & $\begin{array}{l}22 \\
24 \\
25 \\
18 \\
24\end{array}$ & $\begin{array}{l}16 \\
19 \\
21 \\
14 \\
18\end{array}$ & $\begin{array}{l}488 \\
499 \\
319 \\
109 \\
534\end{array}$ \\
\hline
\end{tabular}

Mean number of species identified per sample: $\quad 18$

Mean number of individuals per sample: $\quad 390$

Sampling date: March 8, 1988

$\begin{array}{rrrr}1 & 9 & 8 & 10 \\ 2 & 16 & 9 & 88 \\ 3 & 30 & 17 & 128 \\ 4 & 20 & 14 & 217 \\ 5 & 17 & 11 & 217\end{array}$

Mean number of species identified per sample: $\quad 12$

Mean number of individuals per sample: $\quad 132$

$\begin{array}{ccc}1 & 29 & \\ 2 & 17 \\ 3 & 22 \\ 4 & 22 & \\ 5 & 17 & \\ \text { Mean number of species identified per sample: } & 17 \\ \text { Mean number of individuals per sample: } & 274\end{array}$

Sampling date: May 31, 1988

$22 \quad 359$

$14-171$

$16 \quad 127$

$17-452$

$14 \quad 259$

Mean number of individuals per sample: $\quad 274$

Sampling date: July 28,1988

$\begin{array}{rrrr}1 & 20 & 14 & 398 \\ 2 & 21 & 13 & 156 \\ 3 & 20 & 16 & 57 \\ 4 & 15 & 10 & 125 \\ 5 & 11 & 9 & 142\end{array}$

Mean number of species identified per sample: 12

Mean number of individuals per sample: $\quad 176$

Sampling date: September 13, 1988

$\begin{array}{rrrr}1 & 19 & 11 & 125 \\ 2 & 17 & 11 & 220 \\ 3 & 23 & 14 & 430 \\ 4 & 19 & 16 & 92 \\ 5 & 26 & 19 & 472\end{array}$

Mean number of species identified per sample: $\quad 14$

Mean number of individuals per sample: $\quad 268$

Sampling date: November 1, 1988

$\begin{array}{rrrr}1 & 20 & 15 & 183 \\ 2 & 26 & 20 & 671 \\ 3 & 13 & 8 & 88 \\ 4 & 17 & 11 & 33 \\ 5 & 16 & 12 & 125\end{array}$

Mean number of species identified per sample: 13

Mean number of individuals per sample: $\quad 220$ 
Table 4.-Summary of benthic macrofauna data-Continued

\begin{tabular}{lccc}
\hline $\begin{array}{l}\text { Replicate } \\
\text { No. }\end{array}$ & $\begin{array}{c}\text { Taxonomic } \\
\text { entries }\end{array}$ & $\begin{array}{c}\text { Species } \\
\text { identified }\end{array}$ & Individuals \\
\hline
\end{tabular}

\section{Coyote Point}

\begin{tabular}{llcl}
\multicolumn{4}{c}{ Sampling date: January 13, 1988} \\
1 & 19 & 16 & 427 \\
2 & 20 & 17 & 428 \\
3 & 20 & 17 & 387 \\
4 & 17 & 15 & 299 \\
5 & 16 & 15 & 258
\end{tabular}

Mean number of species identified per sample: 16

Mean number of individuals per sample: $\quad 360$

427

428

299

258

Sampling date: March 8, 1988

$\begin{array}{llll}1 & 23 & 20 & 222 \\ 2 & 21 & 18 & 186 \\ 3 & 17 & 14 & 299 \\ 4 & & & \\ 5 & 16 & 13 & 147\end{array}$

Mean number of species identified per sample: 16

Mean number of individuals per sample: $\quad 214$

$\begin{array}{rrrr} & & \text { Sampling date: May 31, 1988 } & \\ 1 & 20 & 18 & 680 \\ 2 & 17 & 16 & 615 \\ 3 & 23 & 22 & 659 \\ 4 & 23 & 22 & 835 \\ 5 & 25 & 24 & 683\end{array}$

Mean number of species identified per sample: 20

Mean number of individuals per sample: $\quad 694$

Sampling date: July 25,1988

$\begin{array}{rrrr}1 & 17 & 16 & 118 \\ 2 & 22 & 17 & 189 \\ 3 & 20 & 16 & 151 \\ 4 & 26 & 21 & 173 \\ 5 & 13 & 10 & 85\end{array}$

Mean number of species identified per sample: 16

Mean number of individuals per sample: $\quad 143$

\begin{tabular}{llcr}
\multicolumn{4}{c}{ Sampling date: September 14, 1988} \\
1 & 35 & 27 & 1,068 \\
2 & 34 & 28 & 1,053 \\
3 & 36 & 29 & 1,271 \\
4 & 40 & 36 & 1,176 \\
5 & 32 & 25 & 940
\end{tabular}

Mean number of species identified per sample: 29

Mean number of individuals per sample: $\quad 1,102$

Sampling date: November 2, 1988

$\begin{array}{ll}1 & 39 \\ 2 & 35 \\ 3 & 35 \\ 4 & 40 \\ 5 & 35\end{array}$

Méan number of species identified per sample: 29

Mean number of individuals per sample: $\quad 2,496$ 
Table 4.-Summary of benthic macrofauna data-Continued.

\begin{tabular}{lccc}
\hline $\begin{array}{l}\text { Replicate } \\
\text { No. }\end{array}$ & $\begin{array}{c}\text { Taxonomic } \\
\text { entries }\end{array}$ & $\begin{array}{c}\text { Species } \\
\text { identified }\end{array}$ & Individuals \\
\hline
\end{tabular}

\section{Grizzly Bay}

Sampling date: January 11,1988

$\begin{array}{llll}1 & 4 & 3 & 202 \\ 2 & 6 & 5 & 197 \\ 3 & 5 & 4 & 217 \\ 4 & 7 & 6 & 277 \\ 5 & 4 & 3 & 196\end{array}$

Mean number of species identified per sample: 4

Mean number of individuals per sample: $\quad 218$

\begin{tabular}{lrrr}
\multicolumn{4}{c}{ Sampling date: March 14, 1988} \\
1 & 7 & 6 & 177 \\
2 & 6 & 5 & 347 \\
3 & 5 & 4 & 176 \\
4 & 6 & 5 & 253 \\
5 & 5 & 4 & 309
\end{tabular}

Mean number of species identified per sample: 5

Mean number of individuals per sample: $\quad 252$

Sampling date: May 23, 1988

$\begin{array}{llll}1 & 4 & 3 & 162 \\ 2 & 3 & 2 & 157 \\ 3 & 5 & 4 & 186 \\ 4 & 5 & 4 & 151 \\ 5 & 4 & 3 & 225\end{array}$

Mean number of species identified per sample: 3

Mean number of individuals per sample: $\quad 176$

\begin{tabular}{|c|c|c|c|}
\hline \multicolumn{4}{|c|}{ Sampling date: July 21, 1988} \\
\hline 1 & 3 & 3 & 79 \\
\hline 2 & 4 & 3 & 51 \\
\hline 3 & 3 & 3 & 223 \\
\hline 4 & 4 & 3 & 100 \\
\hline 5 & 3 & 3 & 109 \\
\hline
\end{tabular}

Mean number of species identified per sample: 3

Mean number of individuals per sample: $\quad 112$

Sampling date: Scptember 12, 1988

$\begin{array}{llll}1 & 4 & 4 & 186 \\ 2 & 4 & 4 & 145 \\ 3 & 5 & 5 & 167 \\ 4 & 8 & 6 & 224 \\ 5 & 7 & 4 & 260\end{array}$

Mean number of species identified per sample: 5

Mean number of individuals per sample: $\quad 196$

Sampling date: October 31, 1988

1
2
3
4
5

5

$6 \quad 5$

$\begin{array}{ll}6 & 3\end{array}$

$7 \quad 5$

Mcan number of species identified per sample: $\quad 4$

Mean number of individuals per sample: 237 
Table 4.-Summary of benthic macrofauna data-Continued

\begin{tabular}{lccc}
\hline $\begin{array}{c}\text { Replicate } \\
\text { No. }\end{array}$ & $\begin{array}{c}\text { Taxonomic } \\
\text { entries }\end{array}$ & $\begin{array}{c}\text { Species } \\
\text { identified }\end{array}$ & Individuas \\
\hline \multicolumn{4}{c}{ Palo Alto } \\
\multicolumn{4}{c}{ Sampling date: January 14,1988} \\
1 & 19 & 14 & 1,505 \\
2 & 19 & 15 & 2,539 \\
3 & 17 & 13 & 3,219 \\
4 & 17 & 13 & 3,118 \\
5 & 17 & 13 & 3,025
\end{tabular}

Mean number of species identified per sample: 14

Mean number of individuals per sample: $\quad 2,681$

Sampling date: March 7, 1988

$\begin{array}{ll}1 & 21 \\ 2 & 19 \\ 3 & 22 \\ 4 & 20 \\ 5 & 20\end{array}$

21

$19-16$

$22-17$

$20-16$

1,505

3,219

3,118

$\begin{array}{lr}\text { Mean number of species identified per sample: } & 17 \\ \text { Mean number of individuals per sample: } & 1,541\end{array}$

$16 \quad 1,701$

1,408

1,437

1,380

1,779

1,701

Mean number of individuals per sample: $\quad 1,541$

Sampling date: May 26, 1988

$\begin{array}{ll}1 & 27 \\ 2 & 26 \\ 3 & 28 \\ 4 & 25 \\ 5 & 23\end{array}$

27

26

23

5,137

3,635

4,742

5,224

25

23

21

4,470

Mean number of species identified per sample: 22

Mean number of individuals per sample: $\quad 4,642$

Sampling date: July 26,1988

$\begin{array}{ll}1 & 20 \\ 2 & 22 \\ 3 & 21 \\ 4 & 20 \\ 5 & 21\end{array}$

20

22

20

1,572

1,526

1,735

20

19

16

1,978

Mean number of species identified per sample: 18

20

1,743

Mean number of individuals per sample: $\quad 1,711$

Sampling date: September 14, 1988

$\begin{array}{ll}1 & 22 \\ 2 & 34 \\ 3 & 25 \\ 4 & 28 \\ 5 & 28\end{array}$

22

34

19

3,808

4,907

25

28

3,822

28

21

5,630

Mean number of species identified per sample: 23

3,804

Mean number of individuals per sample: $\quad 4,394$

Sampling date: November 2, 1988

$\begin{array}{ll}1 & 19 \\ 2 & 25 \\ 3 & 24 \\ 4 & 20 \\ 5 & 28\end{array}$

19

25

24

20

24

Mean number of species identified per sample: 21

Mean number of individuals per sample: $\quad 3,260$ 
Table 4.-Summary of benthic macrofauna data-Continued.

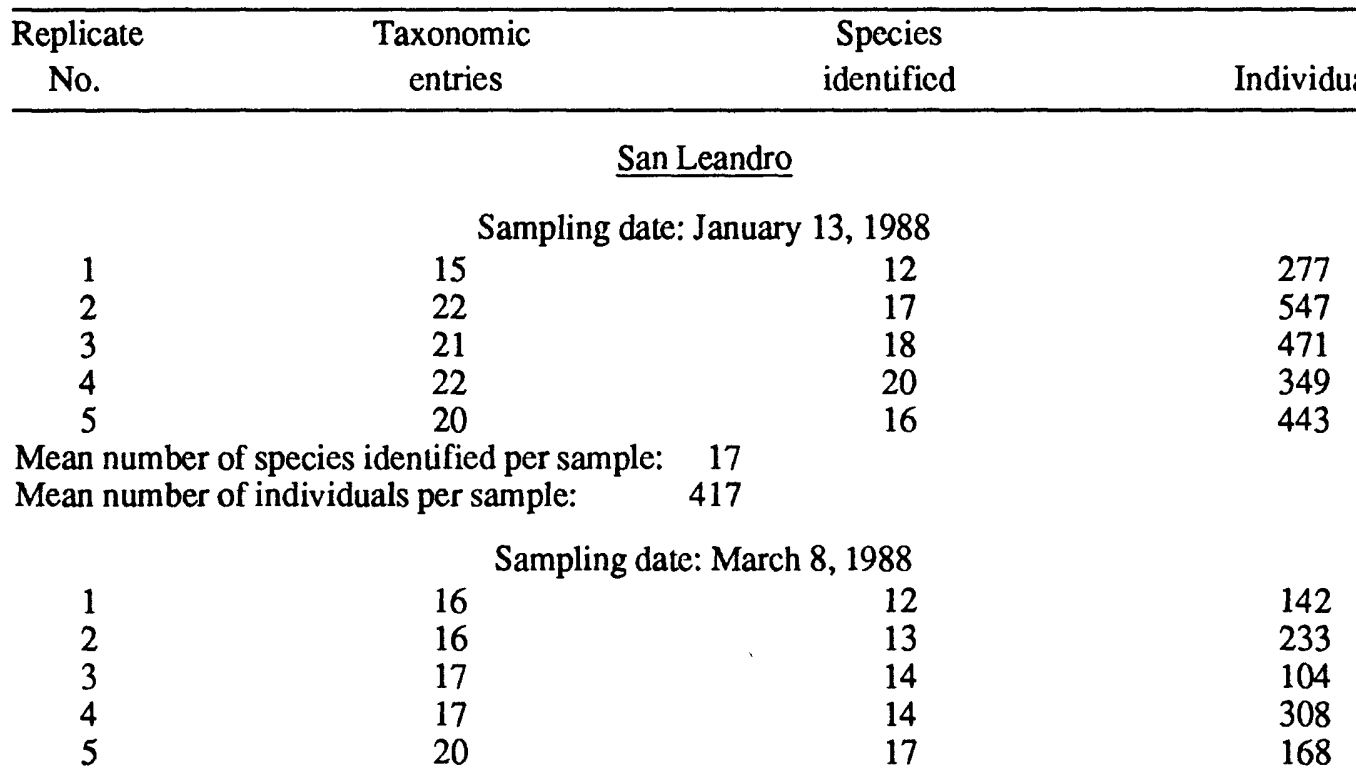

Mean number of species identified per sample: 14

Mean number of individuals per sample: $\quad 191$

Sampling date: May 25, 1988

$\begin{array}{rrrr}1 & 20 & 16 & 123 \\ 2 & 17 & 13 & 246 \\ 3 & 12 & 9 & 63 \\ 4 & 15 & 12 & 144 \\ 5 & 23 & 18 & 319\end{array}$

- Mean number of species identified per sample: 14

Mean number of individuals per sample: $\quad 179$

Sampling date: July 27, 1988

$\begin{array}{rrrr}1 & 10 & 9 & 49 \\ 2 & 16 & 13 & 338 \\ 3 & 10 & 9 & 93 \\ 4 & 13 & 10 & 102 \\ 5 & 12 & 9 & 36\end{array}$

Mean number of species identified per sample: 10

Mean number of individuals per sample: $\quad 124$

Sampling date: Scptember 13, 1988

$\begin{array}{rrrr}1 & 28 & 21 & 1,095 \\ 2 & 24 & 21 & 578 \\ 3 & 27 & 19 & 806 \\ 4 & 26 & 23 & 942 \\ 5 & 28 & 21 & 605\end{array}$

Mean number of species identified per sample: 21

Mean number of individuals per sample: $\quad 805$

49

338

102

36

\begin{tabular}{llcl}
\multicolumn{4}{c}{ Sampling date: November 2, 1988} \\
1 & 20 & 16 & 2,824 \\
2 & 23 & 19 & 3,218 \\
3 & 18 & 16 & 3,541 \\
4 & 23 & 19 & 2,965 \\
5 & 19 & 16 & 2,439
\end{tabular}

Mean number of species identificd per sample: 17

Mean number of individuals per sample: $\quad 2,997$ 
Table 4.-Summary of benthic macrofauna data-Continued.

\begin{tabular}{lccc}
\hline $\begin{array}{l}\text { Replicate } \\
\text { No. }\end{array}$ & Taxonomic & Species & \\
& entries & identified & Individuals \\
\hline
\end{tabular}

\section{San Pablo Deep}

Sampling date: January 12,1988

$\begin{array}{lrrr}1 & 8 & 5 & 95 \\ 2 & 4 & 2 & 96 \\ 3 & 4 & 3 & 120 \\ 4 & 4 & 4 & 64 \\ 5 & 12 & 10 & 186\end{array}$

Mean number of species identified per sample: $\quad 5$

Mean number of individuals per sample: $\quad 112$

Sampling date: March 15, 1988

$\begin{array}{rrrr}1 & 6 & 2 & 76 \\ 2 & 3 & 2 & 66 \\ 3 & 4 & 3 & 58 \\ 4 & 6 & 4 & 107 \\ 5 & 3 & 3 & 35\end{array}$

Mean number of species identified per sample: $\quad 3$

Mean number of individuals per sample: $\quad 68$

$\begin{array}{ccrr} & \text { Sampling date: May 24, } 1988 & \\ 1 & 10 & 7 & 56 \\ 2 & 13 & 10 & 217 \\ 3 & 11 & 8 & 138 \\ 4 & 12 & 10 & 208 \\ 5 & 10 & 8 & 213 \\ \text { ean number of species identified per sample: } & 9 & & \\ \text { Mean number of individuals per sample: } & 166 & & \end{array}$

Mean number of individuals per sample: $\quad 166$

Sampling date: July 20, 1988

\begin{tabular}{rrrr} 
& \multicolumn{2}{c}{ Sampling date: July 20, 1988} & \\
1 & 5 & 4 & 34 \\
2 & 2 & 2 & 23 \\
3 & 9 & 8 & 76 \\
4 & 3 & 2 & 6 \\
5 & 5 & 4 & 26
\end{tabular}

Mean number of species identified per sample: 4

Mean number of individuals per sample: $\quad 33$

Sampling date: September 13, 1988

$\begin{array}{lrrr}1 & 10 & 7 & 179 \\ 2 & 9 & 7 & 127 \\ 3 & 20 & 12 & 245 \\ 4 & 10 & 9 & 178 \\ 5 & 15 & 12 & 252\end{array}$

Mean number of species identified per sample: 9

Mean number of individuals per sample: $\quad 196$

Sampling date: November 1, 1988

$\begin{array}{lrrr}1 & 14 & 11 & 51 \\ 2 & 6 & 4 & 62 \\ 3 & 16 & 11 & 88 \\ 4 & 6 & 5 & 35 \\ 5 & 6 & 5 & 72\end{array}$

Mean number of species identified per sample: 7

Mean number of individuals per sample: $\quad 62$ 
Table 4.-Summary of benthic macrofauna data-Continued.

\begin{tabular}{lccc}
$\begin{array}{c}\text { Replicate } \\
\text { No. }\end{array}$ & $\begin{array}{c}\text { Taxonomic } \\
\text { entries }\end{array}$ & $\begin{array}{c}\text { Species } \\
\text { identified }\end{array}$ & Individuats \\
\hline \multicolumn{4}{c}{ San Pablo Shallow } \\
1 & \multicolumn{2}{c}{ Sampling date: January 12, 1988 } \\
2 & 18 & 14 & 239 \\
3 & 15 & 12 & 294 \\
4 & 12 & 10 & 225 \\
5 & 13 & 12 & 334 \\
5 & 13 & 12 & 170
\end{tabular}

Mean number of species identified per sample: 12

Mean number of individuals per sample: $\quad 252$

Sampling date: March 14, 1988

\begin{tabular}{lrr}
1 & 17 \\
2 & 16 & \\
3 & 16 & \\
4 & 15 & \\
5 & \\
ean number of species identified per sample: & 12 \\
\hline ean number of individuals per sample: & 445
\end{tabular}

$14 \quad 348$

$\begin{array}{ll}12 & 334 \\ 11 & 687\end{array}$

$11 \quad 409$

$\begin{array}{lr}\text { Mean number of species identified per sample: } & 12 \\ \text { Mean number of individuals per sample: } & 445\end{array}$

$\begin{array}{rrrr} & & \text { Sampling datc: May 31, } 1988 & \\ 1 & 16 & 13 & 1,325 \\ 2 & 15 & 12 & 1,647 \\ 3 & 15 & 13 & 978 \\ 4 & 20 & 16 & 1,569 \\ 5 & 17 & 13 & 1,549\end{array}$

Mean number of species identified per sample: 13

Mean number of individuals per sample: $\quad 1,414$

Sampling date: July 25, 1988

$\begin{array}{llll}1 & 15 & 13 & 635 \\ 2 & 18 & 13 & 622 \\ 3 & 14 & 13 & 443 \\ 4 & 17 & 16 & 703 \\ 5 & 15 & 14 & 831\end{array}$

Mean number of species identified per sample: 14

Mean number of individuals per sample: $\quad 647$

Sampling date: September 13, 1988

$\begin{array}{llll}1 & 12 & 12 & 614 \\ 2 & 19 & 13 & 814 \\ 3 & 18 & 15 & 635 \\ 4 & 16 & 14 & 643 \\ 5 & 15 & 13 & 690\end{array}$

Mean number of species identified per sample: 13

Mean number of individuals per sample: $\quad 679$

Sampling date: November 1, 1988

$\begin{array}{crrr}1 & 17 & 13 & 715 \\ 2 & 16 & 12 & 531 \\ 3 & 17 & 14 & 535 \\ 4 & 17 & 13 & 673 \\ 5 & 14 & 8 & 335 \\ \text { Mean number of species identified per sample: } & 12 & & \\ \text { Mean number of individuals per sample: } & 558 & & \end{array}$


Table 4.-Summary of benthic macrofauna data-Continued

\begin{tabular}{|c|c|c|c|}
\hline $\begin{array}{c}\text { Replicate } \\
\text { No. }\end{array}$ & $\begin{array}{c}\text { Taxonomic } \\
\text { entries }\end{array}$ & $\begin{array}{c}\text { Species } \\
\text { identified }\end{array}$ & Individu \\
\hline \multicolumn{4}{|c|}{ South Bay Deep } \\
\hline \multicolumn{4}{|c|}{ Sampling date: January 13,1988} \\
\hline $\begin{array}{l}1 \\
2 \\
3 \\
4 \\
5\end{array}$ & $\begin{array}{l}28 \\
26 \\
30 \\
31 \\
22\end{array}$ & $\begin{array}{l}15 \\
19 \\
19 \\
19 \\
14\end{array}$ & $\begin{array}{l}280 \\
319 \\
415 \\
433 \\
123\end{array}$ \\
\hline
\end{tabular}

Mean number of species identified per sample: 17

Mean number of individuals per sample: $\quad 314$

319

415

433

Sampling date: March 8, 1988

23
28
25
28
23

23

28

16

16

16

16

15

292

295

365

347

234

Mean number of species identified per sample: 16

Mean number of individuals per sample: $\quad 307$

Sampling date: May 25, 1988

20
26
19
26
22

16

21

15

20

20

910

679

607

632

265

Mean number of species identified per sample: 18

Mean number of individuals per sample: $\quad 619$

Sampling date: July 25,1988

$\begin{array}{ll}1 & 24 \\ 2 & 21 \\ 3 & 21 \\ 4 & 30 \\ 5 & 35\end{array}$

24

Mean number of species identified per sample: $\quad 19$

Mean number of individuals per sample:

$$
177
$$

Sampling date: September 14, 1988

$\begin{array}{ll}1 & 28 \\ 2 & 34 \\ 3 & 30 \\ 4 & 30 \\ 5 & 35\end{array}$

28

34

30

35
19

25

21

Mean number of individuals per sample: 204

195

154

239

199

232

Sampling date: November 1,1988

$\begin{array}{ll}1 & 34 \\ 2 & 33 \\ 3 & 40 \\ 4 & 29 \\ 5 & 3\end{array}$

34

$33 \quad 21$

$40-26$

$31 \quad 22$

633

185

Mean number of species identified per sample: 22

Mean number of individuals per sample:

422 


\section{Table 5.-Ancillary data}

[Salinity is reported without traditional units, in accordance with the practical salinity scale of 1978 (Lewis, 1980). Abbreviations: $\mathrm{m}$, meters; ${ }^{\circ} \mathrm{C}$, degrees Celsius, $\mu$ mole $/ \mathrm{L}$, micromoles per liter; $\mathrm{mg} / \mathrm{L}$, milligrams per liter. — , no data]

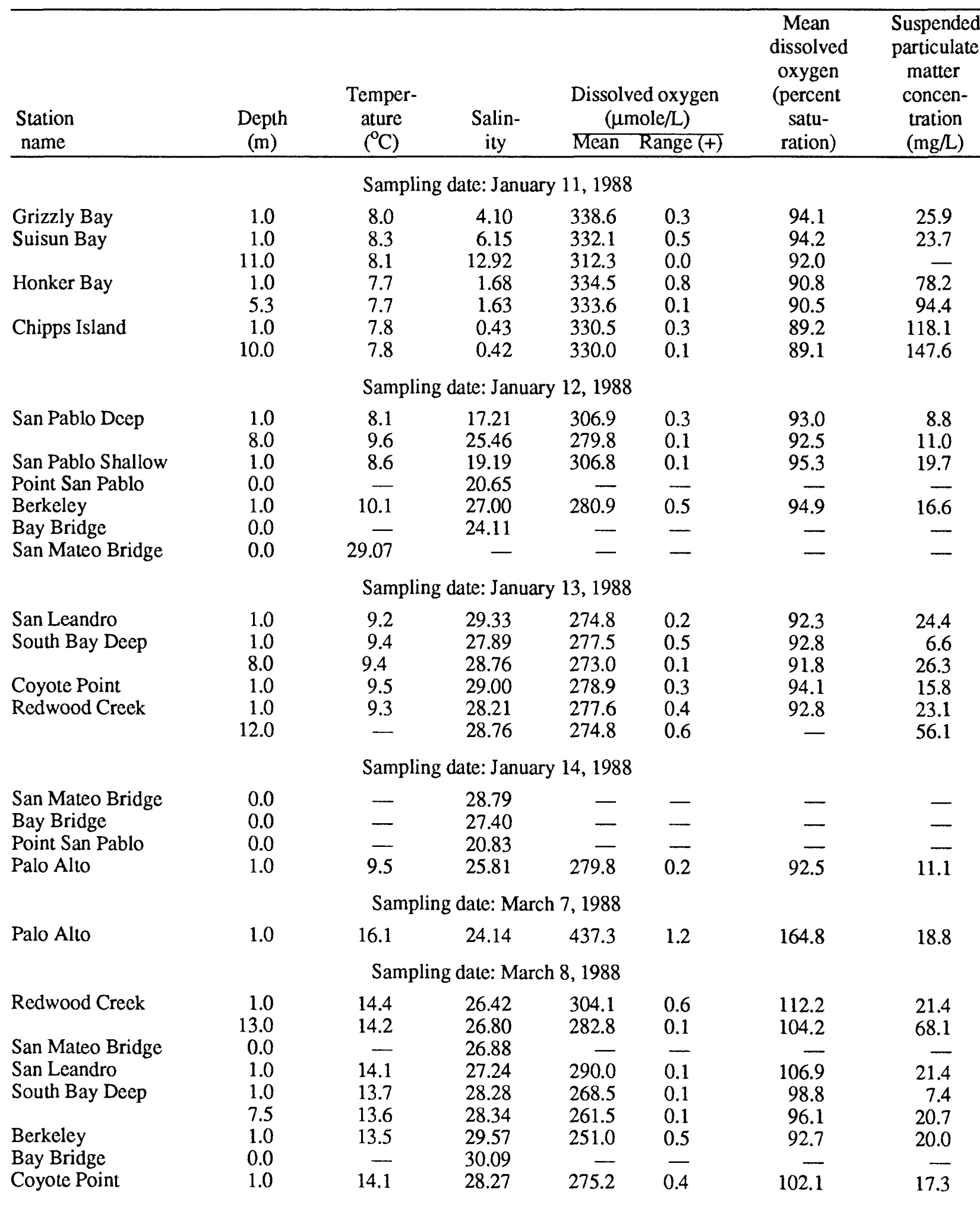


Table 5.-Ancillary data-Continued

\begin{tabular}{|c|c|c|c|c|c|c|c|}
\hline $\begin{array}{l}\text { Station } \\
\text { name }\end{array}$ & $\begin{array}{l}\text { Depth } \\
\text { (m) }\end{array}$ & $\begin{array}{c}\text { Temper- } \\
\text { ature } \\
\left.{ }^{\circ} \mathrm{C}\right)\end{array}$ & $\begin{array}{l}\text { Salin- } \\
\text { ity }\end{array}$ & \multicolumn{2}{|c|}{$\begin{array}{l}\text { Dissolved oxygen } \\
(\mu \mathrm{mole} / \mathrm{L})\end{array}$} & $\begin{array}{l}\text { Mean } \\
\text { dissolved } \\
\text { oxygen } \\
\text { (percent } \\
\text { satu- } \\
\text { ration) }\end{array}$ & $\begin{array}{c}\text { Suspended } \\
\text { particulate } \\
\text { matter } \\
\text { concen- } \\
\text { tration } \\
(\mathrm{mg} / \mathrm{L})\end{array}$ \\
\hline \multicolumn{8}{|c|}{ Sampling date: March 14, 1988} \\
\hline San Mateo Bridge & 0.0 & - & 28.12 & - & - & - & - \\
\hline Bay Bridge & 0.0 & - & 30.68 & - & - & - & - \\
\hline Point San Pablo & 0.0 & - & 28.03 & - & - & - & - \\
\hline San Pablo Shallow & 1.0 & 13.7 & 24.05 & 265.2 & 0.2 & 95.1 & 22.7 \\
\hline Grizzly Bay & 1.0 & 15.0 & 9.46 & 292.6 & 0.3 & 98.6 & 13.9 \\
\hline \multicolumn{8}{|c|}{ Sampling date: March 15, 1988} \\
\hline San Pablo Deep & $\begin{array}{l}1.0 \\
8.5\end{array}$ & $\begin{array}{l}13.2 \\
13.1\end{array}$ & $\begin{array}{l}27.03 \\
27.18\end{array}$ & $\begin{array}{l}258.6 \\
257.6\end{array}$ & $\begin{array}{l}0.1 \\
0.2\end{array}$ & $\begin{array}{l}93.4 \\
92.9\end{array}$ & $\begin{array}{l}42.0 \\
79.6\end{array}$ \\
\hline Suisun Bay & 1.0 & 13.8 & 20.37 & 263.0 & 0.4 & 92.3 & 25.2 \\
\hline & 11.0 & 13.6 & 21.80 & 259.8 & 0.7 & 91.6 & 50.0 \\
\hline Honker Bay & 1.0 & 13.8 & 11.67 & 282.7 & 0.2 & 94.1 & 53.0 \\
\hline & 6.5 & 13.7 & 11.31 & 283.3 & 0.5 & 93.8 & 54.3 \\
\hline Chipps Island & 1.0 & 13.9 & 9.28 & 285.8 & & 94.1 & 46.3 \\
\hline & 13.5 & 13.8 & 11.27 & 279.7 & 0.9 & 92.8 & 47.0 \\
\hline \multicolumn{8}{|c|}{ Sampling date: May 23, 1988} \\
\hline Grizzly Bay & 1.0 & 19.3 & 9.11 & 261.6 & 0.6 & 96.0 & 82.2 \\
\hline \multicolumn{8}{|c|}{ Sampling date: May 24, 1988} \\
\hline Chipps Island & $\begin{array}{l}1.0 \\
8.0\end{array}$ & $\begin{array}{l}19.3 \\
19.1\end{array}$ & 3.60 & 260.0 & 0.4 & 92.3 & 30.9 \\
\hline Honker Bay & 1.0 & $\begin{array}{l}19.1 \\
19.2\end{array}$ & $\begin{array}{l}5.43 \\
6.01\end{array}$ & $\begin{array}{l}254.9 \\
255.7\end{array}$ & $\begin{array}{l}0.1 \\
0.3\end{array}$ & $\begin{array}{l}91.2 \\
92.0\end{array}$ & $\begin{array}{l}89.6 \\
32.6\end{array}$ \\
\hline & 6.0 & 19.2 & 5.88 & 256.1 & 0.7 & 92.0 & 38.0 \\
\hline Suisun Bay & 1.0 & 18.4 & 15.61 & 240.9 & 0.2 & 90.3 & 13.6 \\
\hline & 9.0 & 18.4 & 19.23 & 225.0 & 0.2 & 86.2 & 106.0 \\
\hline San Pablo Deep & 1.0 & 17.9 & 22.29 & 237.6 & 0.3 & 91.8 & 14.0 \\
\hline & 6.5 & - & 26.18 & 227.3 & 0.1 & - & 31.8 \\
\hline Point San Pablo & 0.0 & - & 27.15 & - & - & - & - \\
\hline \multicolumn{8}{|c|}{ Sampling date: May 25, 1988} \\
\hline South Bay Deep & 1.0 & 17.0 & 28.29 & 224.1 & 0.4 & 89.2 & 21.2 \\
\hline & 8.5 & 16.2 & - & 215.5 & 0.5 & 84.6 & 32.1 \\
\hline San Leandro & 1.0 & 17.3 & 29.85 & 233.4 & 0.5 & 93.3 & 37.9 \\
\hline San Mateo Bridge & 0.0 & - & 28.14 & - & 一 & - & 一 \\
\hline \multicolumn{8}{|c|}{ Sampling date: May 26, 1988} \\
\hline Palo Alto & 1.0 & 18.5 & 25.99 & 195.0 & 0.6 & 77.9 & 23.6 \\
\hline Redwood Creek & 1.0 & 18.0 & 29.57 & 220.7 & 0.0 & 89.2 & 21.5 \\
\hline & 12.0 & 17.8 & 29.65 & 219.5 & 0.1 & 88.4 & 69.9 \\
\hline \multicolumn{8}{|c|}{ Sampling date: May 31, 1988} \\
\hline Coyote Point & 1.0 & 16.6 & 30.24 & 236.7 & 0.3 & 93.5 & 106.3 \\
\hline Bay Bridge & 0.0 & - & 31.22 & - & - & - & - \\
\hline Berkeley & 1.0 & - & 30.27 & 238.3 & 0.6 & - & 39.4 \\
\hline Point San Pablo & 0.0 & - & 28.03 & - & - & - & - \\
\hline San Pablo Shallow & 1.0 & 17.4 & 24.65 & 239.6 & 0.1 & 93.0 & 87.5 \\
\hline
\end{tabular}


Table 5.-Ancillary data-Continued

\begin{tabular}{|c|c|c|c|c|c|c|c|}
\hline $\begin{array}{l}\text { Station } \\
\text { name }\end{array}$ & $\begin{array}{c}\text { Depth } \\
\text { (m) }\end{array}$ & $\begin{array}{c}\text { Temper- } \\
\text { ature } \\
\left({ }^{\circ} \mathrm{C}\right) \\
\end{array}$ & $\begin{array}{c}\text { Salin- } \\
\text { ity }\end{array}$ & \multicolumn{2}{|c|}{$\begin{array}{l}\text { Dissolved oxygen } \\
(\mu \mathrm{molc} / \mathrm{L})\end{array}$} & $\begin{array}{l}\text { Mean } \\
\text { dissolved } \\
\text { oxygen } \\
\text { (percent } \\
\text { satu- } \\
\text { ration) } \\
\end{array}$ & $\begin{array}{c}\text { Suspended } \\
\text { particulate } \\
\text { matter } \\
\text { concen- } \\
\text { tration } \\
(\mathrm{mg} / \mathrm{L}) \\
\end{array}$ \\
\hline \multicolumn{8}{|c|}{ Sampling date: July 20, 1988} \\
\hline San Pablo Deep & $\begin{array}{l}1.0 \\
8.5\end{array}$ & $\begin{array}{l}21.7 \\
21.3\end{array}$ & $\begin{array}{l}22.89 \\
25.23\end{array}$ & - & - & - & $\begin{array}{r}35.7 \\
129.0\end{array}$ \\
\hline \multicolumn{8}{|c|}{ Sampling date: July 21,1988} \\
\hline Grizzly Bay & 1.0 & 20.8 & 12.54 & - & - & - & 66.6 \\
\hline \multicolumn{8}{|c|}{ Sampling date: July 25,1988} \\
\hline $\begin{array}{l}\text { Chipps Island } \\
\text { Coyote Point } \\
\text { Honker Bay } \\
\text { Suisun Bay } \\
\text { San Pablo Shallow } \\
\text { Point San Pablo } \\
\text { South Bay Deep }\end{array}$ & $\begin{array}{l}1.0 \\
1.0 \\
1.0 \\
1.0 \\
1.0 \\
0.0 \\
1.0 \\
8.8\end{array}$ & $\begin{array}{r}\overline{21.6} \\
\overline{-} \\
21.0 \\
\overline{21.4} \\
20.5\end{array}$ & $\begin{array}{r}3.90 \\
31.84 \\
5.35 \\
13.91 \\
26.59 \\
29.78 \\
31.84 \\
31.85\end{array}$ & $\begin{array}{l}- \\
z \\
z \\
z\end{array}$ & $\begin{array}{l}z \\
z \\
z \\
z\end{array}$ & $\begin{array}{l}z \\
z \\
z \\
z\end{array}$ & $\begin{array}{r}44.3 \\
21.1 \\
44.1 \\
33.8 \\
48.7 \\
43.6 \\
70.6\end{array}$ \\
\hline \multicolumn{8}{|c|}{ Sampling date: July 26,1988} \\
\hline Palo Alto & 1.0 & 25.1 & 29.53 & - & - & - & 16.3 \\
\hline \multicolumn{8}{|c|}{ Sampling date: July 27, 1988} \\
\hline $\begin{array}{l}\text { Redwood Creek } \\
\text { San Leandro }\end{array}$ & $\begin{array}{r}1.0 \\
11.3 \\
1.0\end{array}$ & $\frac{23.0}{21.8}$ & $\begin{array}{l}31.71 \\
31.93 \\
31.84\end{array}$ & - & - & E & $\begin{array}{l}25.4 \\
44.0 \\
70.7\end{array}$ \\
\hline \multicolumn{8}{|c|}{ Sampling date: July 28,1988} \\
\hline $\begin{array}{l}\text { San Mateo Bridge } \\
\text { Berkeley }\end{array}$ & $\begin{array}{l}0.0 \\
1.0\end{array}$ & $\overline{18.5}$ & $\begin{array}{l}31.90 \\
31.90\end{array}$ & - & - & $\overline{-}$ & $\overline{34.3}$ \\
\hline \multicolumn{8}{|c|}{ Sampling date: September 12,1988} \\
\hline $\begin{array}{l}\text { Grizzly Bay } \\
\text { Suisun Bay } \\
\text { Honker Bay } \\
\text { Chipps Island }\end{array}$ & $\begin{array}{r}1.0 \\
1.0 \\
7.0 \\
1.0 \\
7.5 \\
1.0 \\
12.0\end{array}$ & $\begin{array}{r}19.3 \\
19.1 \\
\overline{1}-\overline{6} \\
19.7 \\
19.7 \\
-\end{array}$ & $\begin{array}{r}12.07 \\
17.62 \\
18.77 \\
9.29 \\
9.52 \\
7.83 \\
9.05\end{array}$ & $\begin{array}{l}255.3 \\
244.0 \\
241.1 \\
254.6 \\
254.6 \\
256.6 \\
257.4\end{array}$ & $\begin{array}{l}0.0 \\
0.3 \\
0.1 \\
0.2 \\
0.7 \\
0.8 \\
1.0\end{array}$ & $\begin{array}{r}95.4 \\
93.9 \\
-\overline{94.1} \\
94.4 \\
94.2 \\
-\end{array}$ & $\begin{array}{l}52.7 \\
51.4 \\
68.6 \\
50.6 \\
69.7 \\
58.4 \\
64.8\end{array}$ \\
\hline \multicolumn{8}{|c|}{ Sampling date: September 13, 1988} \\
\hline $\begin{array}{l}\text { San Pablo Deep } \\
\text { San Pablo Shallow } \\
\text { Point San Pablo } \\
\text { Berkeley } \\
\text { San Leandro }\end{array}$ & $\begin{array}{l}1.0 \\
7.0 \\
1.0 \\
0.0 \\
1.0 \\
1.0\end{array}$ & $\begin{array}{l}18.9 \\
- \\
- \\
-\end{array}$ & $\begin{array}{l}24.78 \\
25.06 \\
25.64 \\
29.15 \\
31.71 \\
32.90\end{array}$ & $\begin{array}{r}234.0 \\
234.6 \\
236.9 \\
- \\
228.9 \\
221.7\end{array}$ & $\begin{array}{l}0.3 \\
0.4 \\
0.2 \\
\overline{0.3} \\
0.0\end{array}$ & $\begin{array}{l}93.6 \\
- \\
-\end{array}$ & $\begin{array}{r}53.5 \\
70.9 \\
88.4 \\
\overline{20.3} \\
18.2\end{array}$ \\
\hline
\end{tabular}


Table 5.-Ancillary data-Continued

\begin{tabular}{|c|c|c|c|c|c|c|c|}
\hline $\begin{array}{l}\text { Station } \\
\text { name }\end{array}$ & $\begin{array}{l}\text { Depth } \\
\text { (m) }\end{array}$ & $\begin{array}{l}\text { Temper- } \\
\text { ature } \\
\left.{ }^{\circ} \mathrm{C}\right)\end{array}$ & $\begin{array}{l}\text { Salin- } \\
\text { ity }\end{array}$ & \multicolumn{2}{|c|}{$\begin{array}{c}\text { Dissolved oxygen } \\
(\mu \mathrm{mole} / \mathrm{L})\end{array}$} & $\begin{array}{c}\text { Mean } \\
\text { dissolved } \\
\text { oxygen } \\
\text { (percent } \\
\text { satu- } \\
\text { ration) }\end{array}$ & $\begin{array}{c}\text { Suspendec } \\
\text { particulate } \\
\text { matter } \\
\text { concen- } \\
\text { tration } \\
(\mathrm{mg} / \mathrm{L})\end{array}$ \\
\hline \multicolumn{8}{|c|}{ Sampling date: September 14,1988} \\
\hline $\begin{array}{l}\text { South Bay Deep } \\
\text { Coyote Point } \\
\text { Palo Alto }\end{array}$ & $\begin{array}{l}1.0 \\
7.5 \\
1.0 \\
1.0\end{array}$ & $\begin{array}{r}19.4 \\
19.3 \\
20.3\end{array}$ & $\begin{array}{l}32.55 \\
32.55 \\
32.62 \\
29.32\end{array}$ & $\begin{array}{l}210.7 \\
210.1 \\
218.9 \\
225.6\end{array}$ & $\begin{array}{l}0.5 \\
0.9 \\
0.1 \\
0.7\end{array}$ & $\begin{array}{r}89.1 \\
-\overline{92.4} \\
95.1\end{array}$ & $\begin{array}{l}19.0 \\
32.0 \\
21.6 \\
10.6\end{array}$ \\
\hline \multicolumn{8}{|c|}{ Sampling date: September 15,1988} \\
\hline $\begin{array}{l}\text { Redwood Creek } \\
\text { San Mateo Bridge } \\
\text { Bay Bridge }\end{array}$ & $\begin{array}{r}1.0 \\
12.0 \\
0.0 \\
0.0\end{array}$ & $\begin{array}{r}20.1 \\
20.1 \\
- \\
-\end{array}$ & $\begin{array}{l}32.47 \\
32.48 \\
32.56 \\
32.23\end{array}$ & $\begin{array}{r}207.8 \\
208.0 \\
- \\
-\end{array}$ & $\begin{array}{l}0.7 \\
0.3 \\
- \\
-\end{array}$ & $\begin{array}{r}88.9 \\
89.0 \\
- \\
-\end{array}$ & $\begin{array}{r}32.6 \\
50.3 \\
- \\
-\end{array}$ \\
\hline \multicolumn{8}{|c|}{ Sampling date: October 31, 1988} \\
\hline $\begin{array}{l}\text { Grizzly Bay } \\
\text { Suisun Bay } \\
\text { Honker Bay } \\
\text { Chipps Island }\end{array}$ & $\begin{array}{r}1.0 \\
1.0 \\
10.0 \\
1.0 \\
6.5 \\
1.0 \\
13.5\end{array}$ & $\begin{array}{r}16.1 \\
16.5 \\
15.7 \\
- \\
= \\
-\end{array}$ & $\begin{array}{r}11.83 \\
15.58 \\
19.40 \\
7.98 \\
11.44 \\
5.55 \\
9.34\end{array}$ & $\begin{array}{l}271.9 \\
265.4 \\
251.3 \\
271.8 \\
265.1 \\
275.4 \\
266.4\end{array}$ & $\begin{array}{l}0.0 \\
0.6 \\
0.3 \\
0.3 \\
0.3 \\
0.3\end{array}$ & $\begin{array}{r}95.1 \\
95.7 \\
91.3 \\
- \\
- \\
-\end{array}$ & $\begin{array}{l}14.7 \\
27.5 \\
46.2 \\
11.8 \\
25.8 \\
11.3 \\
22.7\end{array}$ \\
\hline \multicolumn{8}{|c|}{ Sampling date: November 1,1988} \\
\hline $\begin{array}{l}\text { San Pablo Deep } \\
\text { San Pablo Shallow } \\
\text { Point San Point } \\
\text { Berkeley } \\
\text { South Bay Deep }\end{array}$ & $\begin{array}{l}1.0 \\
7.8 \\
1.0 \\
0.0 \\
1.0 \\
1.0 \\
8.5\end{array}$ & $\begin{array}{r}15.4 \\
15.4 \\
15.6 \\
-\overline{15.1} \\
16.5 \\
16.4\end{array}$ & $\begin{array}{l}28.57 \\
28.86 \\
25.73 \\
27.63 \\
31.44 \\
32.25 \\
32.28\end{array}$ & $\begin{array}{r}235.1 \\
231.5 \\
242.5 \\
- \\
241.6 \\
224.9 \\
218.1\end{array}$ & $\begin{array}{l}0.1 \\
0.5 \\
0.3 \\
- \\
0.7 \\
0.2 \\
0.1\end{array}$ & $\begin{array}{r}89.7 \\
88.5 \\
91.3 \\
93.3 \\
89.7 \\
86.9\end{array}$ & $\begin{array}{r}21.5 \\
38.9 \\
22.3 \\
12.8 \\
8.2 \\
21.1\end{array}$ \\
\hline \multicolumn{8}{|c|}{ Sampling date: November 2, 1988} \\
\hline $\begin{array}{l}\text { Palo Alto } \\
\text { Redwood Creek } \\
\text { San Leandro } \\
\text { Coyote Point }\end{array}$ & $\begin{array}{r}1.0 \\
1.0 \\
14.0 \\
1.0 \\
1.0\end{array}$ & $\begin{array}{l}16.9 \\
16.6 \\
16.6 \\
16.3 \\
16.1\end{array}$ & $\begin{array}{l}30.08 \\
32.33 \\
32.34 \\
32.32 \\
32.48\end{array}$ & $\begin{array}{l}215.1 \\
217.7 \\
215.8 \\
238.6 \\
218.1\end{array}$ & $\begin{array}{l}0.4 \\
0.2 \\
0.0 \\
0.1 \\
0.3\end{array}$ & $\begin{array}{l}85.4 \\
87.1 \\
86.3 \\
94.9 \\
86.4\end{array}$ & $\begin{array}{l}13.8 \\
15.8 \\
28.6 \\
10.4 \\
16.4\end{array}$ \\
\hline \multicolumn{8}{|c|}{ Sampling date: November 3, 1988} \\
\hline $\begin{array}{l}\text { San Mateo Bridge } \\
\text { Bay Bridge }\end{array}$ & $\begin{array}{l}0.0 \\
0.0\end{array}$ & - & $\begin{array}{l}32.42 \\
31.83\end{array}$ & - & - & - & - \\
\hline
\end{tabular}


Table 6.-Summary of sediment grain-size data

[The Phi scale is a logarithmic scale developed to make it easier to directly apply conventional statistical practices to sediment-size data. The equivalent maximum grain sizes in millimeters is shown in parenthesis]

\begin{tabular}{|c|c|c|c|c|c|c|c|c|c|c|}
\hline \multirow[b]{2}{*}{$\begin{array}{c}\text { Sampling } \\
\text { date }\end{array}$} & \multicolumn{10}{|c|}{ Particle-size distribution of bottom material, in percent } \\
\hline & $\begin{array}{c}\text { Phi>0 } \\
(1.0)\end{array}$ & $\begin{array}{l}\text { Phi>1 } \\
(0.50)\end{array}$ & $\begin{array}{l}\text { Phi>2 } \\
(0.25)\end{array}$ & $\begin{array}{l}\text { Phi>3 } \\
(0.125)\end{array}$ & $\begin{array}{l}\text { Phi>4 } \\
(0.062)\end{array}$ & $\begin{array}{l}\text { Phi>5 } \\
(0.031)\end{array}$ & $\begin{array}{l}\text { Phi>6 } \\
(0.016)\end{array}$ & $\begin{array}{l}\text { Phi>7 } \\
(0.008)\end{array}$ & $\begin{array}{l}\text { Phi>8 } \\
(0.004)\end{array}$ & $\begin{array}{c}\text { Phi>9 } \\
(0.002)\end{array}$ \\
\hline \multicolumn{11}{|c|}{ Berkeley } \\
\hline $\begin{array}{l}\text { Jan. } 12,1988 \\
\text { Mar. } 8 \\
\text { May } 31 \\
\text { July } 28 \\
\text { Sept. 13 } \\
\text { Nov. 1 }\end{array}$ & $\begin{array}{l}- \\
z \\
z \\
-\end{array}$ & $\begin{array}{l}\overline{-} \\
\frac{100}{100} \\
100\end{array}$ & $\begin{array}{r}\overline{100} \\
99 \\
100 \\
99 \\
99\end{array}$ & $\begin{array}{r}100 \\
99 \\
94 \\
99 \\
98 \\
92\end{array}$ & $\begin{array}{l}86 \\
90 \\
82 \\
85 \\
85 \\
80\end{array}$ & $\begin{array}{l}74 \\
75 \\
68 \\
72 \\
71 \\
63\end{array}$ & $\begin{array}{l}65 \\
63 \\
59 \\
64 \\
61 \\
53\end{array}$ & $\begin{array}{l}57 \\
53 \\
52 \\
56 \\
53 \\
45\end{array}$ & $\begin{array}{l}53 \\
48 \\
45 \\
46 \\
48 \\
40\end{array}$ & $\begin{array}{l}49 \\
41 \\
38 \\
40 \\
40 \\
34\end{array}$ \\
\hline \multicolumn{11}{|c|}{ Coyote Point } \\
\hline $\begin{array}{l}\text { Jan. } 13,1988 \\
\text { Mar. } 8 \\
\text { May } 31 \\
\text { July } 25 \\
\text { Sept. } 14 \\
\text { Nov. } 2\end{array}$ & $\begin{array}{l}\overline{-} \\
\overline{100} \\
-\end{array}$ & $\begin{array}{r}100 \\
100 \\
99 \\
100 \\
100\end{array}$ & $\begin{array}{r}100 \\
99 \\
99 \\
99 \\
99 \\
99\end{array}$ & $\begin{array}{l}99 \\
98 \\
98 \\
98 \\
97 \\
97\end{array}$ & $\begin{array}{l}95 \\
92 \\
89 \\
94 \\
92 \\
94\end{array}$ & $\begin{array}{l}85 \\
84 \\
84 \\
87 \\
84 \\
86\end{array}$ & $\begin{array}{l}74 \\
73 \\
73 \\
74 \\
73 \\
73\end{array}$ & $\begin{array}{l}65 \\
62 \\
64 \\
65 \\
62 \\
63\end{array}$ & $\begin{array}{l}60 \\
55 \\
55 \\
56 \\
55 \\
58\end{array}$ & $\begin{array}{l}56 \\
47 \\
48 \\
48 \\
48 \\
47\end{array}$ \\
\hline \multicolumn{11}{|c|}{ Grizzly Bay } \\
\hline $\begin{array}{l}\text { Jan. } 11,1988 \\
\text { Mar. 14 } \\
\text { May } 23 \\
\text { July 21 } \\
\text { Sept. } 12 \\
\text { Oct. } 31\end{array}$ & $\begin{array}{l}- \\
z \\
z \\
-\end{array}$ & $\begin{array}{l}- \\
- \\
- \\
-\end{array}$ & $\begin{array}{l}- \\
- \\
- \\
-\end{array}$ & $\begin{array}{l}100 \\
100 \\
100 \\
100 \\
100 \\
100\end{array}$ & $\begin{array}{l}97 \\
98 \\
99 \\
98 \\
99 \\
99\end{array}$ & $\begin{array}{l}92 \\
93 \\
96 \\
95 \\
95 \\
95\end{array}$ & $\begin{array}{l}76 \\
78 \\
87 \\
83 \\
80 \\
81\end{array}$ & $\begin{array}{l}57 \\
62 \\
68 \\
68 \\
63 \\
64\end{array}$ & $\begin{array}{l}51 \\
50 \\
58 \\
54 \\
54 \\
52\end{array}$ & $\begin{array}{l}40 \\
42 \\
48 \\
44 \\
46 \\
43\end{array}$ \\
\hline \multicolumn{11}{|c|}{$\underline{\text { Palo Alto }}$} \\
\hline $\begin{array}{l}\text { Jan. } 14,1988 \\
\text { Mar. } 7 \\
\text { May } 26 \\
\text { July } 26 \\
\text { Sept. } 14 \\
\text { Nov. } 2\end{array}$ & $\begin{array}{l}z \\
z \\
z \\
-\end{array}$ & $\begin{array}{l}100 \\
100 \\
100 \\
100 \\
100 \\
100\end{array}$ & $\begin{array}{l}99 \\
98 \\
98 \\
99 \\
99 \\
99\end{array}$ & $\begin{array}{l}99 \\
97 \\
96 \\
98 \\
97 \\
97\end{array}$ & $\begin{array}{l}95 \\
91 \\
91 \\
93 \\
93 \\
93\end{array}$ & $\begin{array}{l}86 \\
83 \\
84 \\
86 \\
86 \\
86\end{array}$ & $\begin{array}{l}71 \\
71 \\
72 \\
72 \\
73 \\
75\end{array}$ & $\begin{array}{l}63 \\
61 \\
62 \\
61 \\
62 \\
65\end{array}$ & $\begin{array}{l}58 \\
52 \\
54 \\
58 \\
56 \\
57\end{array}$ & $\begin{array}{l}51 \\
44 \\
46 \\
52 \\
47 \\
50\end{array}$ \\
\hline \multicolumn{11}{|c|}{ San Leandro } \\
\hline $\begin{array}{l}\text { Jan. 13, } 1988 \\
\text { Mar. } 8 \\
\text { May } 25 \\
\text { July } 27 \\
\text { Sept. 13 } \\
\text { Nov. } 2\end{array}$ & $\begin{array}{l}- \\
\overline{100} \\
\frac{-}{-}\end{array}$ & $\begin{array}{r}100 \\
100 \\
100 \\
98 \\
100 \\
100\end{array}$ & $\begin{array}{l}99 \\
98 \\
97 \\
96 \\
99 \\
99\end{array}$ & $\begin{array}{l}97 \\
96 \\
91 \\
95 \\
97 \\
95\end{array}$ & $\begin{array}{l}88 \\
85 \\
83 \\
78 \\
87 \\
90\end{array}$ & $\begin{array}{l}79 \\
75 \\
73 \\
71 \\
77 \\
76\end{array}$ & $\begin{array}{l}69 \\
65 \\
62 \\
61 \\
67 \\
66\end{array}$ & $\begin{array}{l}62 \\
55 \\
56 \\
54 \\
57 \\
55\end{array}$ & $\begin{array}{l}58 \\
51 \\
49 \\
47 \\
54 \\
51\end{array}$ & $\begin{array}{l}50 \\
44 \\
42 \\
40 \\
46 \\
45\end{array}$ \\
\hline \multicolumn{11}{|c|}{ San Pablo Deep } \\
\hline $\begin{array}{l}\text { Jan. } 12,1988 \\
\text { Mar. } 15 \\
\text { May } 24 \\
\text { July } 20 \\
\text { Sept. } 13 \\
\text { Nov. } 1\end{array}$ & $\begin{array}{l}- \\
\overline{9} \\
93 \\
-\end{array}$ & $\begin{array}{r}100 \\
100 \\
100 \\
92 \\
90 \\
100\end{array}$ & $\begin{array}{l}99 \\
91 \\
99 \\
75 \\
86 \\
98\end{array}$ & $\begin{array}{l}86 \\
43 \\
93 \\
17 \\
48 \\
91\end{array}$ & $\begin{array}{l}70 \\
37 \\
92 \\
14 \\
37 \\
86\end{array}$ & $\begin{array}{l}64 \\
35 \\
90 \\
13 \\
35 \\
78\end{array}$ & $\begin{array}{l}54 \\
28 \\
84 \\
11 \\
28 \\
65\end{array}$ & $\begin{array}{r}42 \\
22 \\
72 \\
9 \\
24 \\
53\end{array}$ & $\begin{array}{r}34 \\
20 \\
58 \\
8 \\
20 \\
46\end{array}$ & $\begin{array}{r}28 \\
18 \\
47 \\
7 \\
17 \\
37\end{array}$ \\
\hline \multicolumn{11}{|c|}{ San Pablo Shallow } \\
\hline $\begin{array}{l}\text { Jan. } 12,1988 \\
\text { Mar. } 14 \\
\text { May } 31 \\
\text { July } 25 \\
\text { Sept. } 13 \\
\text { Nov. 1 }\end{array}$ & $\begin{array}{l}- \\
z \\
z\end{array}$ & $\begin{array}{l}\overline{100} \\
100 \\
100 \\
100 \\
100\end{array}$ & $\begin{array}{r}100 \\
99 \\
98 \\
98 \\
99 \\
99\end{array}$ & $\begin{array}{l}98 \\
98 \\
95 \\
97 \\
98 \\
98\end{array}$ & $\begin{array}{l}82 \\
84 \\
81 \\
77 \\
86 \\
91\end{array}$ & $\begin{array}{l}75 \\
72 \\
73 \\
69 \\
75 \\
83\end{array}$ & $\begin{array}{l}61 \\
58 \\
61 \\
59 \\
62 \\
70\end{array}$ & $\begin{array}{l}51 \\
48 \\
52 \\
49 \\
51 \\
59\end{array}$ & $\begin{array}{l}44 \\
42 \\
45 \\
41 \\
46 \\
50\end{array}$ & $\begin{array}{l}39 \\
35 \\
39 \\
33 \\
38 \\
41\end{array}$ \\
\hline \multicolumn{11}{|c|}{ South Bay Deep } \\
\hline $\begin{array}{l}\text { Jan. } 13,1988 \\
\text { Mar. } 8 \\
\text { May } 25 \\
\text { July } 25 \\
\text { Sept. } 14 \\
\text { Nov. } 1\end{array}$ & $\begin{array}{l}100 \\
100 \\
100 \\
100 \\
100\end{array}$ & $\begin{array}{r}99 \\
99 \\
98 \\
100 \\
99 \\
99\end{array}$ & $\begin{array}{l}83 \\
97 \\
84 \\
84 \\
88 \\
67\end{array}$ & $\begin{array}{l}55 \\
87 \\
60 \\
64 \\
70 \\
50\end{array}$ & $\begin{array}{l}47 \\
76 \\
53 \\
51 \\
61 \\
44\end{array}$ & $\begin{array}{l}40 \\
65 \\
45 \\
46 \\
55 \\
37\end{array}$ & $\begin{array}{l}33 \\
56 \\
38 \\
38 \\
48 \\
31\end{array}$ & $\begin{array}{l}30 \\
48 \\
33 \\
34 \\
41 \\
26\end{array}$ & $\begin{array}{l}27 \\
43 \\
30 \\
30 \\
36 \\
24\end{array}$ & $\begin{array}{l}24 \\
39 \\
26 \\
25 \\
31 \\
20\end{array}$ \\
\hline
\end{tabular}


Table 7.-Cumulative number of species with respect to number of replicates

\begin{tabular}{|c|c|c|c|c|c|}
\hline \multirow[b]{2}{*}{ Cruise Name } & \multicolumn{2}{|c|}{31601} & \multicolumn{2}{|c|}{ Number of replicates } & \multirow[b]{2}{*}{5} \\
\hline & 1 & 2 & 3 & 4 & \\
\hline \multicolumn{6}{|c|}{ Berkeley } \\
\hline $\begin{array}{l}\text { JAN88 } \\
\text { MAR88 } \\
\text { MAY88 } \\
\text { JUL88 } \\
\text { SEP88 } \\
\text { NOV88 }\end{array}$ & $\begin{array}{r}16 \\
8 \\
22 \\
14 \\
11 \\
15\end{array}$ & $\begin{array}{l}25 \\
13 \\
24 \\
19 \\
15 \\
25\end{array}$ & $\begin{array}{l}29 \\
21 \\
25 \\
26 \\
21 \\
25\end{array}$ & $\begin{array}{l}29 \\
23 \\
29 \\
28 \\
25 \\
31\end{array}$ & $\begin{array}{l}29 \\
26 \\
30 \\
30 \\
30 \\
32\end{array}$ \\
\hline \multicolumn{6}{|c|}{ Coyote Point } \\
\hline $\begin{array}{l}\text { JAN88 } \\
\text { MAR88 } \\
\text { MAY88 } \\
\text { JUL88 } \\
\text { SEP88 } \\
\text { NOV88 }\end{array}$ & $\begin{array}{l}16 \\
20 \\
18 \\
16 \\
27 \\
29\end{array}$ & $\begin{array}{l}19 \\
24 \\
22 \\
21 \\
34 \\
34\end{array}$ & $\begin{array}{l}22 \\
24 \\
27 \\
23 \\
38 \\
38\end{array}$ & $\begin{array}{l}24 \\
31 \\
26 \\
41 \\
45\end{array}$ & $\begin{array}{l}26 \\
26 \\
35 \\
26 \\
41 \\
45\end{array}$ \\
\hline \multicolumn{6}{|c|}{ Grizzly Bay } \\
\hline $\begin{array}{l}\text { JAN88 } \\
\text { MAR88 } \\
\text { MAY88 } \\
\text { JUL88 } \\
\text { SEP88 } \\
\text { NOV88 }\end{array}$ & $\begin{array}{l}3 \\
6 \\
3 \\
3 \\
4 \\
3\end{array}$ & $\begin{array}{l}6 \\
9 \\
3 \\
4 \\
7 \\
5\end{array}$ & $\begin{array}{l}7 \\
9 \\
4 \\
5 \\
7 \\
6\end{array}$ & $\begin{array}{l}8 \\
9 \\
5 \\
6 \\
9 \\
8\end{array}$ & $\begin{array}{l}8 \\
9 \\
5 \\
6 \\
9 \\
8\end{array}$ \\
\hline \multicolumn{6}{|c|}{$\underline{\text { Palo Alto }}$} \\
\hline $\begin{array}{l}\text { JAN88 } \\
\text { MAR88 } \\
\text { MAY88 } \\
\text { JUL88 } \\
\text { SEP88 } \\
\text { NOV88 }\end{array}$ & $\begin{array}{l}14 \\
18 \\
24 \\
17 \\
19 \\
17\end{array}$ & $\begin{array}{l}16 \\
20 \\
25 \\
21 \\
28 \\
24\end{array}$ & $\begin{array}{l}16 \\
20 \\
27 \\
24 \\
29 \\
27\end{array}$ & $\begin{array}{l}16 \\
21 \\
28 \\
24 \\
31 \\
27\end{array}$ & $\begin{array}{l}16 \\
21 \\
28 \\
25 \\
31 \\
30\end{array}$ \\
\hline
\end{tabular}


Table 7.-Cumulative number of species with respect to number of replicates-Continued

\begin{tabular}{|c|c|c|c|c|c|}
\hline \multirow[b]{2}{*}{ Cruise name } & \multicolumn{5}{|c|}{ Number of replicates } \\
\hline & 1 & 2 & 3 & 4 & 5 \\
\hline \multicolumn{6}{|c|}{$\underline{\text { San Leandro }}$} \\
\hline $\begin{array}{l}\text { JAN88 } \\
\text { MAR88 } \\
\text { MAY88 } \\
\text { JUL88 } \\
\text { SEP88 } \\
\text { NOV88 }\end{array}$ & $\begin{array}{r}12 \\
12 \\
16 \\
9 \\
21 \\
16\end{array}$ & $\begin{array}{l}20 \\
15 \\
18 \\
16 \\
24 \\
20\end{array}$ & $\begin{array}{l}22 \\
18 \\
18 \\
18 \\
25 \\
20\end{array}$ & $\begin{array}{l}25 \\
19 \\
20 \\
20 \\
28 \\
23\end{array}$ & $\begin{array}{l}27 \\
23 \\
26 \\
21 \\
29 \\
23\end{array}$ \\
\hline \multicolumn{6}{|c|}{ San Pablo Deep } \\
\hline $\begin{array}{l}\text { JAN88 } \\
\text { MAR88 } \\
\text { MAY88 } \\
\text { JUL88 } \\
\text { SEP88 } \\
\text { NOV88 }\end{array}$ & $\begin{array}{r}5 \\
2 \\
7 \\
4 \\
7 \\
11\end{array}$ & $\begin{array}{r}5 \\
2 \\
11 \\
5 \\
9 \\
12\end{array}$ & $\begin{array}{r}6 \\
4 \\
13 \\
11 \\
14 \\
15\end{array}$ & $\begin{array}{r}8 \\
6 \\
15 \\
12 \\
15 \\
15\end{array}$ & $\begin{array}{r}12 \\
8 \\
16 \\
14 \\
17 \\
15\end{array}$ \\
\hline \multicolumn{6}{|c|}{ San Pablo Shallow } \\
\hline $\begin{array}{l}\text { JAN88 } \\
\text { MAR88 } \\
\text { MAY88 } \\
\text { JUL88 } \\
\text { SEP88 } \\
\text { NOV88 }\end{array}$ & $\begin{array}{l}14 \\
14 \\
13 \\
13 \\
12 \\
13\end{array}$ & $\begin{array}{l}15 \\
\\
14 \\
17 \\
15 \\
17\end{array}$ & $\begin{array}{l}15 \\
15 \\
20 \\
19 \\
19 \\
19\end{array}$ & $\begin{array}{l}15 \\
17 \\
23 \\
21 \\
19 \\
21\end{array}$ & $\begin{array}{l}16 \\
18 \\
24 \\
21 \\
21 \\
22\end{array}$ \\
\hline \multicolumn{6}{|c|}{ South Bay Deep } \\
\hline $\begin{array}{l}\text { JAN88 } \\
\text { MAR88 } \\
\text { MAY88 } \\
\text { JUL88 } \\
\text { SEP88 } \\
\text { NOV88 }\end{array}$ & $\begin{array}{l}15 \\
16 \\
16 \\
16 \\
19 \\
22\end{array}$ & $\begin{array}{l}22 \\
22 \\
24 \\
21 \\
29 \\
28\end{array}$ & $\begin{array}{l}27 \\
25 \\
25 \\
22 \\
30 \\
36\end{array}$ & $\begin{array}{l}28 \\
26 \\
28 \\
30 \\
35 \\
40\end{array}$ & $\begin{array}{l}30 \\
27 \\
32 \\
35 \\
41 \\
41\end{array}$ \\
\hline
\end{tabular}

